\title{
Beyond food and medicine, but necessary for life, too: other folk plant uses in several territories of Catalonia and the Balearic Islands
}

Airy Gras ${ }^{1}$, Teresa Garnatje $2^{*}$, M. Àngels Bonet ${ }^{1}$, Esperança Carrió ${ }^{1}$, Marina Mayans ${ }^{1}$, Montse Parada ${ }^{1}$, Montse Rigat ${ }^{1}$ and Joan Vallès ${ }^{1}$

\begin{abstract}
Background: Ethnobotanical academic research, particularly in European industrialised countries, has been, and is, mostly focused on folk uses of food and medicinal plants. Nevertheless, other uses, as may well be supposed, account for a significant portion of these folk uses. In the Catalan linguistic domain, a considerable amount of ethnobotanical work has been produced, but to date almost nothing has been published on these other plant uses.

Methods: We basically used the method of semistructured interviews to collect data on names, knowledge and use of plants in the above-mentioned fields from 759 informants in three Catalonian (Alt Empordà, Montseny and Ripollès) and two Balearic (Formentera and Mallorca) areas. We identified the plants quoted by the informants and prepared herbarium vouchers. We analysed and compared the results obtained.

Results: Information has been collected on 401 genera, 552 species, 81 subspecies and four varieties, belonging to 122 families, totalling 4137 use reports for popular non-food and non-medicinal uses (classified in 14 modalities), and designated with 1303 folk Catalan names. The informant consensus factor is 0.87 , accounting for a consistent and robust dataset.

Conclusion: Contrarily to what could be thought a priori, and irrespective of the fact that some uses are declining or changing, non-medicinal and non-food folk plant uses strongly persist in the territories considered, are highly considered by their practitioners, and may even imply some economic revenues.
\end{abstract}

Keywords: Balearic Islands, Catalonia, Ethnobotany, Non-food and non-medicinal plant uses

\section{Background}

When coining the term ethnobotany, Harshberger [1] considered as basic points for the newly named science "elucidating the cultural position of tribes who used plants" (where, nowadays, 'tribes' is replaced by 'human groups', [2]), "clarifying the past distribution of plants", "determining trade routes" and "suggesting new current production lines" for useful plants. For Portères and Barrau [3, 4], ethnobotany is a discipline located at the

\footnotetext{
* Correspondence: tgarnatje@ibb.csic.es

${ }^{2}$ Institut Botànic de Barcelona (IBB-CSIC-ICUB), Passeig del Migdia s/n. Parc

de Montjuïc, 08038 Barcelona, Catalonia, Spain

Full list of author information is available at the end of the article
}

crossroad between natural and human sciences studying the behaviour of human societies with regard to plants. Other authors state that ethnobotanical research rescues and updates the history of plants in human societies through time and space [5].

Even if ethnobotany deals with all kinds of plant uses, at least in western European countries, ethnobotanical research focused on food and medicinal plants is by far the most dominant, what can be verified when surveying the articles that have appeared in relevant journals. Such a situation can be explained by two kinds of reasons. On the one hand, food and medicine are the two plant folk applications more directly linked to human health, so 
that they are maintained at a relatively high rate even in industrialised societies. Conversely, these countries have undergone a rather deep process of acculturation, in the sense of adopting so-called modern habits in detriment of traditional culture [6], which implied that rural communities abandoned a great deal of traditional practices linked to plants, because they are much less necessary (if necessary at all) nowadays than in the first half of $20^{\text {th }}$ century. On the other hand, the above-quoted food and medicinal properties are those which most likely can lead to the development of new commercial sources of welfare products (see e.g., [7]). Some non-food and nonmedicinal popular uses are slightly better preserved than most of them, such as basketry [8] and cosmetics $[9,10]$, the latter being, in fact, very closely linked to medicinal ones. Some specific papers with ethnobotanical focus have been devoted to these other plant uses in European countries (e.g., those quoted for basketry and cosmetics, as well as [11-16]). Additionally, some compilation work, rather addressed to folk knowledge vulgarisation, contain information on these plant utilisations; as an example the first volume of the ongoing Spanish Inventory of Traditional Knowledge related to Biodiversity [17] provides information on all kinds of plant uses, including those dealt with in the present paper. In any case, even taking into account the above-quoted contributions, this approach still remains quite scarce.

The research on ethnobotany in the Catalan linguistic domain has been intense in the last 25 years. This research mostly comprises data on food and medicinal (including veterinarian) plant uses, as well as studies linked to agroecosystems (mostly homegardens) and to ethnoecological questions (cf. [10, 18], and references therein). Only very rarely other plant uses have been addressed $[19,20]$, although, throughout our research, we have maintained the conviction that a robust pool of knowledge on these uses still exists. Taking this into account, the aims of the present paper are: 1) to provide an overview on non-food and non-medicinal popular plant uses in five Catalan language territories, three in Catalonia and two in the Balearic Islands; 2) to evaluate to what extent these uses are persisting and how are they currently considered by their practitioners.

\section{Methods}

\section{Study areas}

We performed interviews in five territories located in two large areas of the Catalan linguistic domain, three in Catalonia (Alt Empordà, Montseny, Ripollès) and two in the Balearic Islands (Formentera, Mallorca), with the aim of comparing a continental place and an insular one (Fig. 1). The Catalonian areas comprise from plain to high mountains, and the Balearic ones involve two islands.
Alt Empordà (AE) is a district (comarca in the Catalan language) of $1358 \mathrm{~km}^{2}$ and 140,214 inhabitants [21] living in 68 municipalities. The climate is mainly coastal Mediterranean. The vegetation is distributed in an asymmetrical form in two biogeographical regions, Mediterranean, largely dominant, and Eurosiberian, in certain mountainous areas, reaching $1443 \mathrm{~m}$ a.s.l.

Ripollès (RI) is a district occupying an area of $956.6 \mathrm{~km}^{2}$ and having a population of 25,700 inhabitants [21] distributed in 19 municipalities, with a high percentage of the population inhabiting small villages and isolated houses. Located in the eastern Pyrenees, it has a high mountain climate with Mediterranean influence. The flora and vegetation are mostly Eurosiberian, with some Boreoalpine zones in mountain areas reaching $2909.8 \mathrm{~m}$ a.s.l. Montseny (MO) is a mountain massif with a maximum altitude of $1706 \mathrm{~m}$ a.s.l., with an area of $826 \mathrm{~km}^{2}$ and a population of 105,000 people [22]. The climate is basically Mediterranean (including mountain Mediterranean). The vegetation belongs to the Mediterranean and Eurosiberian regions. Formentera (FO) is the smallest of the four inhabited Balearic Islands, occupying $82 \mathrm{~km}^{2}$, and has 11,545 inhabitants [23] living in nine population centres, all belonging to one municipality. Its maximum altitude is $195 \mathrm{~m}$. The climate is Mediterranean with an arid tendency. The vegetation landscape is basically coastal Mediterranean.

Mallorca (MA) is the biggest island in the Balearic archipelago, and the seventh largest in the Mediterranean sea, with the highest altitude at $1445 \mathrm{~m}$. It has an extension of $3622.54 \mathrm{~km}^{2}$ and population of 858,313 inhabitants [23]. Its climate is typically Mediterranean. The vegetation belongs mostly to the Mediterranean and, to a small part, to the Eurosiberian biogeographic regions.

\section{Informants}

Information was obtained from 769 informants, selected on a snowball basis [24] for general ethnobotanical prospections in each of the areas studied. Of them, 510 were from Catalonia and 259 from Balearic Islands, distributed as follows: 160 from RI, born between 1915 and 1988, with an average age of 71.6 years; 178 from $\mathrm{AE}$, with a mean age of 69.44 years, ranging from 23 to $95 ; 172$ from MO, with a mean age of 66 , ranging from 31 to $96 ; 235$ from MA, with an average of 76 years, born between 1906 and 1981; and 24 from FO, born between 1920 and 1943. Concerning gender, 445 interviewees were women $(57.87 \%)$ and 324 men $(42.13 \%)$.

\section{Field work methods}

Data were collected with the same method, but in different years in each territory: 1993-2000 (MO), 1995-2007 


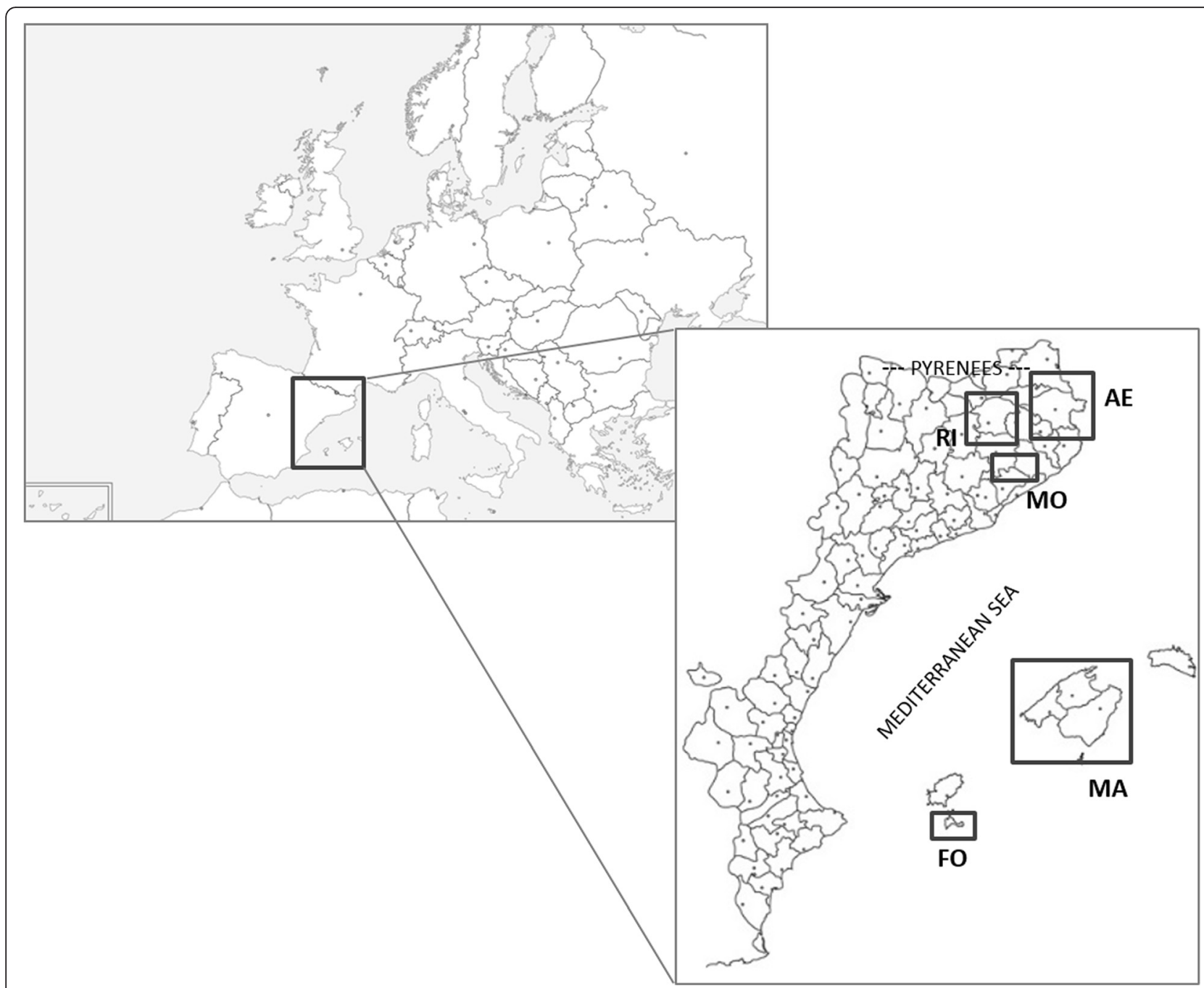

Fig. 1 Studied areas in the contexts of Europe and the Catalan linguistic domain. AE: Alt Empordà; MO: Montseny; Rl: Ripollès; MA: Mallorca; FO: Formentera

(AE), 2004-2012 (RI), 2009-2013 (MA) and 2011-2013 (FO). Ethnobotanical interviews were performed with a total of 769 informants (see Results and discussion section for details on distribution, gender and age). Taking into account the Code of Ethics of the International Society of Ethnobiology [25], we asked the interviewees' informed consent to participate in the survey, to register the interviews, to take pictures and to use their images and information. The method used was basically the semistructured interview [26], with special care taken in not asking direct questions, which could coerce, condition or influence the informant's answer. In several occasions group interviews were carried out, separating the answers of the different interviewees. Whenever possible, we collected the plants in question together with the informants or we observed with them those they had preserved at home or their preparations or the objects elaborated with them. The interviews, developed in Catalan, the common language of both interviewers and interviewees, were registered, also with the informants' agreement (which was also solicited for taking pictures).

Plants were identified at specific or infraspecific levels with the aid of the Flora manual dels Països Catalans [27], and for family assignation we followed the Angiosperm Phylogeny Group ([28]; http://www.mobot.org/ MOBOT/research/APweb/), as currently reflected in The plant list (http://www.theplantlist.org), also devoted to gymnosperms and ferns. Vouchers of every taxon with associated information have been prepared, collected following the legislation and avoiding protected areas, and deposited in the herbaria $\mathrm{BCN}$, of the Centre de Documentació de Biodiversitat Vegetal, Universitat de Barcelona, and $\mathrm{BC}$, of the Institut Botànic de Barcelona. 


\section{Databasing}

Once in the laboratory, the interviews were transcribed and introduced into our research team's database (www.etnobotanica.cat, not open to public access, but punctually consultable on request to the corresponding or to the last authors). This database contains all ethnobotanical data (on medicinal, food and other uses) from our group and allows us to manage the results, to perform some calculations and to establish comparisons. Taking Cook's Economic Botany data standard as a departing point [29], a huge effort to establish and nuance use categories (as well as parts of plants and kinds of preparation and use, the latter not relevant here, but in medicinal and food uses) has been performed, and it is reflected in Table 1 .

\section{Statistical methods}

All calculations were carried out using Excel (Microsoft Excel 2007) and the program XLSTAT (v. 2007.5, Addinsoft SARL) was used to carry out the Chi-square test in order to check the statistical differences in the use categories among the four studied territories.

\section{Results and discussion}

\section{General data}

The 769 informants reported 401 genera (34 of the taxa reported have only been determined to the generic level), 552 species, 81 subspecies and four varieties, belonging to 122 families. The taxa collected, their folk names and uses, the territories where they are recorded, the parts of plants employed and their use frequency are presented in Table 1, placed as an appendix. The number of taxa with such information is high. Just to compare with the same geographical areas, 334 species are reported in a paper on medicinal plants (AE, [30]), which is representative of other territories, a figure higher than in other areas and not simply additive, since a high number of taxa are repeated in different areas, and 97 species were recorded in a work on ethnoveterinary in four out of the five territories here considered [31]. This supports the argument to consider the nonfood and non-medicinal folk plant uses as not secondary or residual at all. The number of use reports (hereinafter UR) confirms this, being also elevated (4137 in total, cf. Table 2 for their distribution in the geographical areas and Table 3 for its repartition for use categories).

To name the specific and infraspecific taxa the informants provided 1303 Catalan popular names, including whole plants and parts of plant, especially fruits and seeds, and elaborated products. This figure would be the equivalent to a mean of more than two names per plant, and roughly approaches the $5 \%$ of the ca. 35,000 Catalan plant names (of folk and other provenances) recently collected [32], the quantity and diversity of names accounting too for the vitality of the knowledge of plants with these other uses. Additionally, the informants indicated 37 Spanish, four French and one Arabic names to refer to the plants reported.

\section{Use categories}

Table 3 presents the 14 categories (which are equally present for all territories in our database to avoid comparison) of non-food and non-medicinal uses in which we have classified all data collected. Some examples and comments on specific uses are provided in this and in other epigraphs of this paper (e.g., most reported taxa or persistence of uses), and the detail of all uses collected can be found in Table 1.

The category "other informations" is a miscellaneous one to hold sparse knowledge not attributable to the established categories. This big group of information comprises 509 use reports from a total 4137, meaning ca. $12 \%$ of all reports, but includes a very numerous and diverse cases, for which we have established the following subgrouping: ecological information, undesirable actions, purchased in commerce, harvesting and/or selling, excipient or adjuvant in medicinal or food preparations, product appreciations. We also attributed the category of other information to the cases in which the informants declared that they knew that a plant was useful, but they did not remember for what (use not remembered); we maintain this category (related, as the whole paper, to non-food and non-medicinal uses) as an evocation of knowledge that used to be solid, but has declined and is almost forgotten now.

Some big categories comprise several activities. Agrosilvopastoral: agricultural/horticultural, for pig slaughter, honey obtaining, for fishing, for hunting, forestry tools for agricultural practices, weeds. Artisanal: elaboration of canes, shoes, brooms, kitchen implements, musical instruments, baskets and similar objects, fibres, smoking paper, tobacco pipes and other instruments, as well as hide tanning. Domestic: household help, air fresheners, paint elaboration, help in sewing. Textile: fibre or cloth elaboration, textile padding, dyer. Timber: elaboration of furniture, boats and wheeled vehicles, obtention of building material.

With this, and irrespective of our grouping, made for the sake of concision, thus, the number of subcategories (including categories with no subcategories) would reach 46, showing a big coverage of different fields. The most common large use categories, in terms of number of use reports, are ornamental (646 use reports), agrosilvopastoral management (600), artisanal (534), and magic and religious beliefs and practices (421). For statistical purposes we used the main or big categories, since for many of the subcategories there are not enough use reports to allow a statistical treatment. 
Table 1 Non-food and non-medicinal folk plant uses in the territories of Alt Empordà, Montseny and Ripollès (Catalonia), and Formentera and Mallorca (Balearic Islands)

\begin{tabular}{|c|c|c|c|c|c|}
\hline Taxon & $\begin{array}{l}\text { Catalan vernacular names } \\
\text { (a few Spanish, French and } \\
\text { Arabic, indicated) }\end{array}$ & Area & Used part & Uses & Frequency \\
\hline $\begin{array}{l}\text { Abelmoschus esculentus (L.) } \\
\text { Moench (Malvaceae) } \\
\text { BC131944 }\end{array}$ & Ocra & $\mathrm{Bl}$ & Seed & $\begin{array}{l}\text { Agrosilvopastoral management: agricultural/ } \\
\text { horticultural }\end{array}$ & 1 \\
\hline $\begin{array}{l}\text { Abies alba Mill. } \\
\text { (Pinaceae) BCN3096 }\end{array}$ & Avet & $A E, M O$ & $\begin{array}{l}\text { Aerial part. } \\
\text { Stem. } \\
\text { Whole plant }\end{array}$ & $\begin{array}{l}\text { Magic and religious beliefs and practices } \\
\text { Ornamental: bouquet elaboration } \\
\text { Timber: boat manufacturing }\end{array}$ & 3 \\
\hline $\begin{array}{l}\text { Abies nordmanniana } \\
\text { (Steven) Spach (Pinaceae) } \\
\text { BC59249 }\end{array}$ & Avet & MO & Whole plant & Ornamental: gardening & 1 \\
\hline $\begin{array}{l}\text { Abies ×masjoanis D.A. Soto, } \\
\text { J.I.G. Viñas et E. Bujarrabal } \\
\text { (Pinaceae) BCN35720 }\end{array}$ & $\begin{array}{l}\text { Avet de Masjoan, avet } \\
\text { del Montseny }\end{array}$ & MO & Whole plant & Ornamental: gardening & 3 \\
\hline $\begin{array}{l}\text { Acacia dealbata Link } \\
\text { (Mimosaceae) BCN29973 }\end{array}$ & Mimosa & $A E, M O$ & Aerial part. Leaf & $\begin{array}{l}\text { Magic and religious beliefs and practices } \\
\text { Ornamental: bouquet elaboration }\end{array}$ & 3 \\
\hline $\begin{array}{l}\text { Acer campestre L. } \\
\text { (Aceraceae) BCN29613 }\end{array}$ & Auró, auró blanc & $\mathrm{AE}, \mathrm{RI}$ & $\begin{array}{l}\text { Stem. Whole } \\
\text { plant }\end{array}$ & $\begin{array}{l}\text { Ludic } \\
\text { Timber: furniture manufacturing }\end{array}$ & 2 \\
\hline $\begin{array}{l}\text { Acer monspessulanum L. } \\
\text { (Aceraceae) BCN29613 }\end{array}$ & Auró negre & $A E$ & Stem & Ludic & 1 \\
\hline $\begin{array}{l}\text { Acer opalus Mill. subsp. } \\
\text { opalus (Aceraceae) } \\
\text { BCN29615 }\end{array}$ & Blada, baladre & $\mathrm{AE}, \mathrm{MO}$ & $\begin{array}{l}\text { Stem. Whole } \\
\text { plant }\end{array}$ & $\begin{array}{l}\text { Agrosilvopastoral management: agricultural/ } \\
\text { horticultural } \\
\text { Folk oral literature } \\
\text { Ludic }\end{array}$ & 4 \\
\hline $\begin{array}{l}\text { Achillea ageratum L. } \\
\text { (Asteraceae) } \\
\text { BCN- E-188 }\end{array}$ & Alè de bou & $\mathrm{Bl}$ & $\begin{array}{l}\text { Aerial part. } \\
\text { Whole plant }\end{array}$ & $\begin{array}{l}\text { Agrosilvopastoral management: tools for } \\
\text { agricultural practices } \\
\text { Magic and religious beliefs and practices } \\
\text { Other informations: ecological information }\end{array}$ & 3 \\
\hline $\begin{array}{l}\text { Achillea millefolium L. } \\
\text { (Asteraceae) BCN29616 }\end{array}$ & Centfulles & $A E$ & Aerial part & Ornamental: bouquet elaboration & 1 \\
\hline $\begin{array}{l}\text { Achillea ptarmica L. subsp. } \\
\text { pyrenaica (Godr.) Heimerl } \\
\text { (Asteraceae) BCN24701 }\end{array}$ & $\begin{array}{l}\text { Camamilla, camamilla } \\
\text { de muntanya, camamilla } \\
\text { de Rojà }\end{array}$ & $\mathrm{RI}$ & Aerial part & Cosmetic & 1 \\
\hline $\begin{array}{l}\text { Adiantum capillus-veneris L. } \\
\text { (Pteridiaceae) BCN91889 }\end{array}$ & Falzia & $\mathrm{Bl}$ & $\begin{array}{l}\text { Not reported. } \\
\text { Whole plant }\end{array}$ & $\begin{array}{l}\text { Cosmetic } \\
\text { Other informations: excipient or adjuvant in } \\
\text { medicinal or food preparations } \\
\text { Other informations: use not remembered }\end{array}$ & 3 \\
\hline $\begin{array}{l}\text { Aegilops geniculata Roth } \\
\text { (Poaceae) BCN } 103555\end{array}$ & Blat de perdiu & $\mathrm{Bl}$ & $\begin{array}{l}\text { Aerial part. } \\
\text { Whole plant }\end{array}$ & Other informations: ecological information & 2 \\
\hline $\begin{array}{l}\text { Aesculus hippocastanum L. } \\
\text { (Sapindaceae) BCN29618 }\end{array}$ & $\begin{array}{l}\text { Castanyer bord, } \\
\text { castanyer d'Índies, } \\
\text { castanya agra, castanya } \\
\text { borda (fruit), castanya de } \\
\text { castanyer d'Índies (fruit), } \\
\text { morronyer }\end{array}$ & $\begin{array}{l}\mathrm{AE}, \mathrm{BI} \\
\mathrm{MO}, \mathrm{RI}\end{array}$ & $\begin{array}{l}\text { Fruit. Seed. } \\
\text { Whole plant }\end{array}$ & $\begin{array}{l}\text { Ludic } \\
\text { Magic and religious beliefs and practices } \\
\text { Ornamental: gardening }\end{array}$ & 8 \\
\hline $\begin{array}{l}\text { Agapanthus sp. } \\
\text { (Amaryllidaceae) }\end{array}$ & Acapantus & $\mathrm{Bl}$ & Whole plant & Ornamental: gardening & 1 \\
\hline $\begin{array}{l}\text { Agave americana } \mathrm{L} \text {. } \\
\text { (Agavaceae) BCN46860 }\end{array}$ & $\begin{array}{l}\text { Ágave, atzavara, } \\
\text { donarda, figuerassa, } \\
\text { figuerassa de marge, } \\
\text { figuerassa grossa, pita, } \\
\text { pitera, punta de pita } \\
\text { (thorn), punyalera, } \\
\text { xeremec (stem) }\end{array}$ & $\mathrm{AE}, \mathrm{BI}, \mathrm{MO}$ & $\begin{array}{l}\text { Flower. Leaf, } \\
\text { Not reported. } \\
\text { Stem. Thorn }\end{array}$ & $\begin{array}{l}\text { Agrosilvopastoral management: agricultural/ } \\
\text { horticultural } \\
\text { Artisanal: cookware elaboration } \\
\text { Artisanal: footwear elaboration } \\
\text { Domestic: for help at home } \\
\text { Textile: fibre or cloth elaboration } \\
\text { Timber: construction materials production }\end{array}$ & 18 \\
\hline Agrimonia eupatoria L. & Agafallosa & $\mathrm{Rl}$ & Fruit & Ludic & 3 \\
\hline
\end{tabular}


Table 1 Non-food and non-medicinal folk plant uses in the territories of Alt Empordà, Montseny and Ripollès (Catalonia), and Formentera and Mallorca (Balearic Islands) (Continued)

Agrostemma githago L.
(Caryophyllaceae) BCN5737
Ailanthus altissima (Mill.)
Swingle (Simaroubaceae)
BCN81396
Ajuga iva (L.) Schreb.
(Lamiaceae) BCN6437
Alkanna tinctoria (L.) Tausch
(Boraginaceae) BCN24706
Allium ampeloprasum L.
(Amaryllidaceae) BCN3993
Allium cepa L. (Liliaceae)
BCN28655

Allium fistulosum L. (Amaryllidaceae) BCN-E248

Allium porrum L. (Amaryllidaceae) BCN28791

Allium roseum L. (Amaryllidaceae) BCN31259

Allium sativum L. (Amaryllidaceae) BCN29832
Allium triquetrum $\mathrm{L}$. (Amaryllidaceae) BCN95566

Alnus glutinosa (L.) Gaertn. Vern (Betulaceae) BCN29620
Clavell de blat, niella

Cornissa, irlando

Iva

Herba del peu de colom,

peu de colom, pota de colom

All de bruixa, allassa, porrerell

Ceba, ceba forastera, grell

$\begin{array}{ll}A E, B I, M O & \text { Bulb. } \\ \text { Inflorescence }\end{array}$

Porro

All de bruixa, calabruix

All, all de Sant Pau, all pau

$\mathrm{MO}, \mathrm{RI}$

$\mathrm{MO}$

$\mathrm{BI}$

RI

$\mathrm{AE}$,

$A E, B I$

$\mathrm{AE}$

$\mathrm{AE}, \mathrm{BI}, \mathrm{MO}$ Bulb.
Cebollí

Bl Inflorescence

Inflorescence. Whole plant

Bl Whole plant

$A E, M O, R I$ Aerial part. Stem. Whole plant

$\mathrm{AE}, \mathrm{BI} \quad$ Whole plant

Alocasia odora (Lindl.)

Aloe vera (L.) Burm f. (Xanthorrhoeaceae)

\section{BCN27242}

Althaea rosea (L.) Cav. (Malvaceae) BCN6470

Alyssum maritimum (L.) Lam. (Brassicaceae) BCN29622

$\mathrm{BI}, \mathrm{MO}$

Flower. Whole

plant

AE, BI, RI Whole plant
K.Koch (Araceae) BCN50215

\section{Marquesa}

Aloe vera, àloe vera, cactus, figuerassa

Malva reial, vauma, vauma loca

Alíssum, caps blancs, escopinya de Crist, flor de gat, herbamel
Magic and religious beliefs and practices Other informations: ecological information

Agrosilvopastoral management: honey obtaining

Domestic: for help at home

Magic and religious beliefs and practices

Ornamental: bouquet elaboration

Other informations: ecological information Repellent

Ornamental: bouquet elaboration

Agrosilvopastoral management: agricultural/ horticultural

Ornamental: bouquet elaboration

Other informations: ecological information

Agrosilvopastoral management: agricultural/ 2 1 1 2 horticultural

Folk oral literature

Magic and religious beliefs and practices

Ornamental : gardening

Ornamental: bouquet elaboration

Other informations: ecological information

Other informations: ecological information

Agrosilvopastoral management: for fishing Agrosilvopastoral management: for hunting Agrosilvopastoral management: tools for agricultural practices

Artisanal: footwear elaboration

Folk oral literature

Magic and religious beliefs and practices Ornamental: bouquet elaboration

Magic and religious beliefs and practices Ornamental: gardening

Other informations: ecological information

Leaf juice. Not Cosmetic

reported. Magic and religious beliefs and practices

Whole plant Other informations: ecological information

Magic and religious beliefs and practices Ornamental: gardening

Agrosilvopastoral management: tool for agricultural practices

Magic and religious beliefs and practices Other informations: ecological information Other informations: use not remembered Repellent 
Table 1 Non-food and non-medicinal folk plant uses in the territories of Alt Empordà, Montseny and Ripollès (Catalonia), and Formentera and Mallorca (Balearic Islands) (Continued)

\begin{tabular}{lll}
\hline Ampelodesmos mauritanicus & Càrritx, càrritx femella, & B \\
(Poir.) T.Durand \& Schinz & càrritx mascle, carritxera
\end{tabular}

(Poaceae) BCN95589

\begin{abstract}
Anacyclus clavatus (Desf.)
Pers. (Asteraceae)

BCN31260

Anacyclus valentinus $\mathrm{L}$.

(Asteraceae) BCN29625

Anagallis arvensis L. subsp.

arvensis (Primulaceae)

BCN19974

Anagyris foetida $\mathrm{L}$.

(Fabaceae) BCN16938

Anastatica hierochuntica L. (Brassicaceae) BCN16949

\section{Anemone coronaria $\mathrm{L}$.}

(Ranunculaceae) BCN17399

Anemone pulsatilla $\mathrm{L}$.

(Ranunculaceae) BCN24711

Anethum graveolens L.

(Apiaceae) BCN83632

Anthemis arvensis L.

(Asteraceae) BC840033

Anthemis cotula L.

(Asteraceae) BCN29835

Anthyllis cytisoides L.

(Fabaceae) BCN20700
\end{abstract}

Antirrhinum majus L. (Plantaginaceae) BCN46074

Aphyllanthes monspelliensis

L. (Asparagaceae)

BCN29627

Apium graveolens L. (Apiaceae) BCN46859

Aquilegia vulgaris L. (Ranunculaceae) BCN27253

Arachis hypogaea L.

(Fabaceae) BCN46858

Araucaria heterophylla

(Salisb.) Franco

(Araucariaceae) BCN67780

Araujia sericifera Brot.

(Apocynaceae) BCN299975

Arbutus unedo L. (Ericaceae) BCN100976

B।

Aerial part.

Leaf. Stem.

Whole plant

Bòlig

Bòlig

Borró

Garrover del dimoni

Rosa de Jericó

Anèmones

Flor de Sant Pere

Anet

Bòlig, sistorna

Camamilla borda

Aubada, botja de cuques

Badocs, boques de conill,

conillets, gatets, gossets,

gossos, gorges de llop,

mamaconills, pets de Ilop

Llonzella

Àpit

Campaneta

Cacauet

Arbre de pisos

Miraguano

Arboç, arboça (fruit),

arbocera, cirera d'arboç

(fruit), Ilipota (fruit), llipoter

AE Whole plant

AE Whole plant

Aerial part

RI Whole plant

BI Whole plant

RI Aerial part

BI $\quad$ Aerial part

AE, MO, RI Aerial part

plant

AE

Aerial part

plant

RI Aerial part

BI Whole plant

AE $\quad$ Fruit

$\mathrm{BI}, \mathrm{MO} \quad$ Aerial part.

Flower. Fruit.
Agrosilvopastoral management: for pig

18

slaughter

Artisanal: basketry

Other informations: ecological information

Other informations: undesirable actions

Textile: fibre or cloth elaboration

Other informations: ecological information

Other informations: ecological information

2

AE $\quad$ Whole plant

Other informations: ecological information

Other informations: undesirable actions

1

Bl Whole plant

Other informations: use not remembered

1

BI Aerial part Ornamental: bouquet elaboration

1

Other informations: ecological information

2

Ornamental: gardening

1

Ornamental: bouquet elaboration

1

AE Whole plant Other informations: ecological information

1

Agrosilvopastoral management: for pig

5

Artisanal: broom elaboration

Other informations: ecological information

Ludic

Flower. Whole Magic and religious beliefs and practices

Ornamental: bouquet elaboration

Ornamental: gardening

Ludic

Leaf. Whole Other informations: ecological information

Ornamental: bouquet elaboration

Domestic: for help at home

2

Fuel obtaining: charcoal

Ornamental: gardening

Textile: textile padding

Not reported.

Stem. Whole

plant
Agrosilvopastoral management: forestry

Agrosilvopastoral management: honey

obtaining

Fuel obtaining: charcoal

Ornamental: bouquet elaboration

Ornamental: gardening

Other informations: ecological information

Timber 
Table 1 Non-food and non-medicinal folk plant uses in the territories of Alt Empordà, Montseny and Ripollès (Catalonia), and Formentera and Mallorca (Balearic Islands) (Continued)

\begin{tabular}{|c|c|c|c|c|c|}
\hline $\begin{array}{l}\text { Arctium minus (Hill) Bernh. } \\
\text { (Asteraceae) BCN31262 }\end{array}$ & $\begin{array}{l}\text { Enganxadora, Ilapassa, } \\
\text { Ilapissera, gafarró, poltre, } \\
\text { repalassa }\end{array}$ & $\mathrm{AE}, \mathrm{MO}, \mathrm{RI}$ & $\begin{array}{l}\text { Leaf. } \\
\text { Inflorescence. } \\
\text { Infructescence. } \\
\text { Whole plant }\end{array}$ & $\begin{array}{l}\text { Agrosilvopastoral management: tool for } \\
\text { agricultural practices } \\
\text { Ludic } \\
\text { Other informations: ecological information }\end{array}$ & 8 \\
\hline $\begin{array}{l}\text { Arisarum vulgare } \\
\text { O.Targ.Tozz. (Araceae) } \\
\text { BCN95564 }\end{array}$ & $\begin{array}{l}\text { Frare llec, rapa, rapa de } \\
\text { frare }\end{array}$ & $\mathrm{Bl}$ & $\begin{array}{l}\text { Inflorescence. } \\
\text { Whole plant }\end{array}$ & $\begin{array}{l}\text { Folk oral literature } \\
\text { Ludic } \\
\text { Other informations: ecological information }\end{array}$ & 7 \\
\hline $\begin{array}{l}\text { Artemisia abrotanum L. } \\
\text { (Asteraceae) BCN31263 }\end{array}$ & $\begin{array}{l}\text { Absenta, abrótano macho } \\
\text { (Spanish), artemisia, donzell, } \\
\text { estragó, shiba (Arabic) }\end{array}$ & $\mathrm{Bl}$ & Not reported & Cosmetic & 1 \\
\hline $\begin{array}{l}\text { Artemisia absinthium L. } \\
\text { (Asteraceae) BCN29837 }\end{array}$ & $\begin{array}{l}\text { Absenta, abrótano macho } \\
\text { (Spanish), artemisia, encens, } \\
\text { donzell, estragó, shiba } \\
\text { (Arabic) }\end{array}$ & $\mathrm{AE}, \mathrm{Bl}, \mathrm{RI}$ & $\begin{array}{l}\text { Aerial part. } \\
\text { Whole plant }\end{array}$ & $\begin{array}{l}\text { Domestic: air freshener } \\
\text { Domestic: for help at home } \\
\text { Repellent }\end{array}$ & 3 \\
\hline $\begin{array}{l}\text { Artemisia arborescens L. } \\
\text { (Asteraceae) BCN29630 }\end{array}$ & Artemisia, donzell & $\mathrm{AE}, \mathrm{Bl}$ & $\begin{array}{l}\text { Aerial part. } \\
\text { Whole plant }\end{array}$ & $\begin{array}{l}\text { Agrosilvopastoral management: tool for } \\
\text { agricultural practices } \\
\text { Ornamental: bouquet elaboration } \\
\text { Repellent }\end{array}$ & 7 \\
\hline $\begin{array}{l}\text { Artemisia dracunculus L. } \\
\text { (Asteraceae) BCN13326 }\end{array}$ & Estragó & $\mathrm{Bl}$ & Whole plant & Other informations: ecological information & 1 \\
\hline $\begin{array}{l}\text { Artemisia verlotiorum } \\
\text { Lamotte (Asteraceae) } \\
\text { BCN29633 }\end{array}$ & Artemisa, donzell & $A E$ & Aerial part & Magic and religious beliefs and practices & 1 \\
\hline $\begin{array}{l}\text { Artemisia vulgaris L. } \\
\text { (Asteraceae) BCN24719 }\end{array}$ & Altimiris & $\mathrm{Rl}$ & Aerial part & $\begin{array}{l}\text { Agrosilvopastoral management: agricultural/ } \\
\text { horticultural }\end{array}$ & 1 \\
\hline $\begin{array}{l}\text { Arum italicum Mill. } \\
\text { (Araceae) BCN32356 }\end{array}$ & Xàrria & MO & Spathe & Ludic & 1 \\
\hline $\begin{array}{l}\text { Arundo donax L. (Poaceae) } \\
\text { BCN29825 }\end{array}$ & $\begin{array}{l}\text { Canya, canya de torrent, } \\
\text { canya mallorquina, canya } \\
\text { verda, canyeta, canyís }\end{array}$ & $\begin{array}{l}\mathrm{AE}, \mathrm{Bl} \\
\mathrm{MO}, \mathrm{RI}\end{array}$ & $\begin{array}{l}\text { Aerial part. } \\
\text { Leaf. Root. } \\
\text { Stem. Whole } \\
\text { plant }\end{array}$ & $\begin{array}{l}\text { Agrosilvopastoral management: for fishing } \\
\text { Agrosilvopastoral management: for hunting } \\
\text { Agrosilvopastoral management: for pig } \\
\text { slaughter } \\
\text { Agrosilvopastoral management: tools for } \\
\text { agricultural practices } \\
\text { Artisanal: basketry } \\
\text { Artisanal: broom elaboration } \\
\text { Artisanal: cane elaboration } \\
\text { Artisanal: cookware elaboration } \\
\text { Artisanal: musical instrument elaboration } \\
\text { Artisanal: smoking pipe elaboration } \\
\text { Domestic: for help at home } \\
\text { Domestic: for sewing } \\
\text { Fuel obtaining: firewood } \\
\text { Ludic } \\
\text { Magic and religious beliefs and practices } \\
\text { Ornamental: gardening } \\
\text { Other informations: ecological information } \\
\text { Timber: construction materials production }\end{array}$ & 104 \\
\hline $\begin{array}{l}\text { Asparagus acutifolius L. } \\
\text { (Asparagaceae) BCN29976 }\end{array}$ & $\begin{array}{l}\text { Espàrrec (turion), } \\
\text { esparreguera }\end{array}$ & $\mathrm{AE}, \mathrm{BI}, \mathrm{MO}$ & $\begin{array}{l}\text { Aerial part. } \\
\text { Leaf. Stem. } \\
\text { Whole plant }\end{array}$ & $\begin{array}{l}\text { Agrosilvopastoral management: agricultural/ } \\
\text { horticultural } \\
\text { Artisanal: broom elaboration } \\
\text { Magic and religious beliefs and practices }\end{array}$ & 4 \\
\hline $\begin{array}{l}\text { Asparagus albus } \mathrm{L} \text {. } \\
\text { (Asparagaceae) BCN56215 }\end{array}$ & $\begin{array}{l}\text { Espàrrec (turion), } \\
\text { esparreguera }\end{array}$ & $\mathrm{Bl}$ & Aerial part & Ornamental: bouquet elaboration & 1 \\
\hline $\begin{array}{l}\text { Asparagus horridus L. } \\
\text { (Asparagaceae) BCN103541 }\end{array}$ & $\begin{array}{l}\text { Espàrrec (turion), } \\
\text { esparreguera, esparreguera } \\
\text { vera }\end{array}$ & $\mathrm{Bl}$ & Flower & $\begin{array}{l}\text { Agrosilvopastoral management: honey } \\
\text { obtaining }\end{array}$ & 1 \\
\hline $\begin{array}{l}\text { Asparagus sprengeri Regel } \\
\text { (Asparagaceae) BCN57323 }\end{array}$ & Esparreguera de jardí & $\mathrm{Bl}$ & Whole plant & Ornamental: gardening & 1 \\
\hline
\end{tabular}


Table 1 Non-food and non-medicinal folk plant uses in the territories of Alt Empordà, Montseny and Ripollès (Catalonia), and Formentera and Mallorca (Balearic Islands) (Continued)

\begin{tabular}{ll}
\hline Asphodelus aestivus Brot. & Albó, asfodel, aubó, \\
(Xanthorrhoeaceae) & caramutxa, coió de \\
BCN52957 & porrassa, moneiato de \\
& porrassera, porrassa, \\
& porrassa femella, porrassera, \\
& porassó
\end{tabular}

Aspidistra elatior Blume (Asparagaceae) BCN104271

\begin{abstract}
Aspidosperma polyneuron Müll.Arg. (Apocynaceae) BCN52088
\end{abstract}

Asplenium trichomanes $\mathrm{L}$. (Aspleniaceae) BCN24723

Aster novi-belgii L. (Asteraceae) BCN98570

Aster pilosus Willd.

(Asteraceae)

BCN47723

Astragalus balearicus Chater (Fabaceae) BCN95554

Avena barbata Pott ex Link (Poaceae) BCN49867

Avena sativa L. (Poaceae) BCN32185

Avena sterilis L. (Poaceae) BCN52787

Ballota hirsuta Benth. (Lamiaceae) BCN72714

Begonia sp. (Begoniaceae)

Bellis annua L. (Asteraceae) BCN61139

Bellis perennis $\mathrm{L}$. (Asteraceae) BCN31264

Berberis vulgaris L. (Berberidaceae) BCN27274

Beta vulgaris L. subsp. maritima (L.) Arcang.

(Amaranthaceae)

BCN103432

Betula pendula Roth (Betulaceae) BCN29647
Cossiol de fulla, cossiol de $\mathrm{Bl} \quad$ Whole plant saló, fulla de saló

Palorosa

AE

Stem

Auredelleta, costella de paret

Setembrina

Octubre

Coixinet de monja

Cugula, cugula borda, civada borda

Civada

$\mathrm{BI}, \mathrm{MO}$

BI Whole plant

$\mathrm{AE}, \mathrm{BI}, \mathrm{MO}$ Aerial part Fruit. Inflorescence. Stem

Fruit. Whole plant

Cugula, cugula borda,

$\mathrm{BI}, \mathrm{MO}$ cugula vertadera

Sacramentària

BI

Begònia

Confitera

Margarida, margarida

borda, margaridoia,

margarita

Berbèlia

Bledera salvatge

Beç, bedoll

$A E, R I$ Inflorescence. Stem. Whole

plant

Calyx

Whole plant

Whole plant

AE, MO, RI Aerial part. Inflorescence. Whole plant

Fruit wer. Leaf.

Stem. Tuber.

Whole plant

Agrosilvopastoral management: for pig

slaughter

Agrosilvopastoral management: honey

obtaining

Agrosilvopastoral management: tools for agricultural practices

Artisanal: footwear elaboration

Domestic: for help at home

Folk oral literature

Fuel obtaining: firewood

Magic and religious beliefs and practices

Other information: harvesting and/or selling

Other informations: ecological information

Other information: use not remembered

Ornamental: gardening

Artisanal: musical instrument elaboration

Cosmetics

1

Ornamental: gardening

2

Ornamental: gardening

1

Other informations: ecological information

1

Artisanal: musical instrument elaboration

Ludic

Agrosilvopastoral management: agricultural/

9 horticultura

Magic and religious beliefs and practices

Ornamental: bouquet elaboration

Other informations: ecological information

Agrosilvopastoral management: agricultural/

4

horticultural

Artisanal: musical instrument elaboration

Ludic

Domestic: for help at home

1

Ornamental: gardening

5

Other informations: ecological information

1

Magic and religious beliefs and practices

mental: bouquet elaboration

Other informations: ecological information

Ludic

1

Ornamental: bouquet elaboration

Artisanal: broom elaboration

Artisanal: cookware elaboration

Magic and religious beliefs and practices

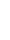

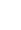

(1)


Table 1 Non-food and non-medicinal folk plant uses in the territories of Alt Empordà, Montseny and Ripollès (Catalonia), and Formentera and Mallorca (Balearic Islands) (Continued)

Bidens tripartita L.
(Asteraceae) BCN50219
Bignonia sp. (Bignoniaceae)
Borago officinalis L.
(Boraginaceae) BCN68582
Bougainvillea sp.
(Nyctaginaceae)
Brachypodium retusum
(Pers.) P.Beauv. (Poaceae)
BCN31265
Brassica napus L.
(Brassicaceae) BCN24727
Brassica oleracea L. subsp.
oleracea (Brassicaceae)
BCN32181
Briza maxima L. (Poaceae)
BCN29648

Bromus hordeaceus L. (Poaceae) BCN52583

Buxus balearica Lam. (Buxaceae) BCN21410

Buxus sempervirens L. (Buxaceae) BCN29843

Pixallits
Coral
Borraja (Spanish), borratxa
Boquemvíl.lia
Llistó
Nap
Col
Arrecadetes de la Mare de
Dellugues, llagrimetes de la
sempreviva
Arrecades de la Mare de
Déu, belluguets, herbe
tremblante French, herba
Auda

Cua de mula

Boix

$\mathrm{BI}, \mathrm{R}$

BI

AE

Aerial part

Ornamental: bouquet elaboration

Whole plant

Ornamental: gardening

Aerial part.

Flower. Whole plant

$\mathrm{BI}$

Flower

AE

Stem. Whole plant

Bl, RI Bulb. Root

Leaf

AE

Aerial part

$A E, M O, R I$ Aerial part

Ornamental: bouquet elaboration agricultural practices

Fuel obtaining: firewood

Other informations: ecological information

Magic and religious beliefs and practices

Folk oral literature

Other informations: use not remembered

Ornamental: bouquet elaboration

4

Boix, boix de jardí, boj (Spanish), olleta
$\mathrm{AE}, \mathrm{BI}$,

$\mathrm{MO}, \mathrm{RI}$

Aerial part

Cortical

parenchyma.

Flower. Fruit

Leaf. Stem.

Whole plant.
Calendula arvensis M.Bieb. (Asteraceae) BCN29637

Calendula officinalis L. (Asteraceae) BCN29977
Llevamà, goig bord, jaumet $A E, B I$

Boixac, bojac, calèndula,

$\mathrm{AE}, \mathrm{BI}$, galdiró, llevamà, llevamà de $\mathrm{MO}, \mathrm{Rl}$ jardí, jaumet lower. Leaf. plant

Artisanal: cane elaboration

Artisanal: cookware elaboration

Ornamental: gardening

Other informations: harvesting and/or selling

Other informations: ecological information

Timber

Agrosilvopastoral management: for pig

slaughter

Agrosilvopastoral management: tools for agricultural practices

Artisanal: broom elaboration

Artisanal: cane elaboration

Artisanal: cookware elaboration

Artisanal: musical instrument elaboration

Artisanal: smoking pipe elaboration

Domestic: for help at home

Fuel obtaining: charcoal

Ludic

Magic and religious beliefs and practices

Ornamental: bouquet elaboration

Ornamental: gardening

Other informations: use not remembered

Aerial part. Not Agrosilvopastoral management: tools for reported.Whole agricultural practices

plant

Cosmetic

Folk oral literature

Other informations: use not remembered

Aerial part.

Inlforescence.

Not reported.

Whole plant
Cosmetic

Magic and religious beliefs and practices

Ornamental: bouquet elaboration

Ornamental: gardening 
Table 1 Non-food and non-medicinal folk plant uses in the territories of Alt Empordà, Montseny and Ripollès (Catalonia), and Formentera and Mallorca (Balearic Islands) (Continued)

\begin{tabular}{|c|c|c|c|c|c|}
\hline $\begin{array}{l}\text { Calicotome spinosa (L.) Link } \\
\text { (Fabaceae) BCN29638 }\end{array}$ & Argelac, argelaga & $A E, B I$ & $\begin{array}{l}\text { Aerial part. } \\
\text { Stem. Whole } \\
\text { plant }\end{array}$ & $\begin{array}{l}\text { Agrosilvopastoral management: agricultural/ } \\
\text { horticultural } \\
\text { Agrosilvopastoral management: for pig } \\
\text { slaughter } \\
\text { Ornamental: gardening }\end{array}$ & 7 \\
\hline $\begin{array}{l}\text { Callistephus chinensis (L.) } \\
\text { Ness (Asteraceae) BCN-E249 }\end{array}$ & Sol coronat & $\mathrm{Bl}$ & Whole plant & Ornamental: gardening & 1 \\
\hline $\begin{array}{l}\text { Calluna vulgaris (L.) Hull } \\
\text { (Ericaceae) BCN29639 }\end{array}$ & Brossa, bruguerola, xerpó & $\mathrm{AE}, \mathrm{MO}, \mathrm{Rl}$ & $\begin{array}{l}\text { Aerial part. } \\
\text { Flower }\end{array}$ & $\begin{array}{l}\text { Agrosilvopastoral management: honey } \\
\text { obtaining } \\
\text { Ornamental: bouquet elaboration }\end{array}$ & 13 \\
\hline $\begin{array}{l}\text { Camellia sinensis (L.) Kuntze } \\
\text { (Theaceae) BCN50762 }\end{array}$ & $\mathrm{Te}$ & $\mathrm{Bl}$ & Not reported & Other informations: ecological information & 1 \\
\hline $\begin{array}{l}\text { Campanula patula L. } \\
\text { (Campanulaceae) BCN29640 }\end{array}$ & Campaneta & $\mathrm{AE}$ & Aerial part & Ornamental: bouquet elaboration & 1 \\
\hline $\begin{array}{l}\text { Campanula persicifolia L. } \\
\text { (Campanulaceae) BCN29641 }\end{array}$ & Campaneta & $\mathrm{AE}$ & Aerial part & Ornamental: bouquet elaboration & 1 \\
\hline $\begin{array}{l}\text { Cannabis sativa L. } \\
\text { (Cannabaceae) BCN24735 }\end{array}$ & $\begin{array}{l}\text { Cànem, cànyem, cànyom, } \\
\text { gansalla (elaborated } \\
\text { product), maria, marihuana }\end{array}$ & $\begin{array}{l}\mathrm{AE}, \mathrm{Bl} \\
\mathrm{MO}, \mathrm{RI}\end{array}$ & $\begin{array}{l}\text { Aerial part. } \\
\text { Inflorescence. } \\
\text { Stem. Whole } \\
\text { plant }\end{array}$ & $\begin{array}{l}\text { Agrosilvopastoral management: for fishing } \\
\text { Agrosilvopastoral management: tools for } \\
\text { agricultural practices } \\
\text { Artisanal: basketry } \\
\text { Artisanal: footwear elaboration } \\
\text { Other information: ecological information } \\
\text { Smoking } \\
\text { Textile: fibre or cloth elaboration } \\
\text { Timber: boat manufacturing }\end{array}$ & 17 \\
\hline $\begin{array}{l}\text { Capparis spinosa L. subsp. } \\
\text { spinosa (Capparaceae) } \\
\text { BCN97808 }\end{array}$ & $\begin{array}{l}\text { Tàpera (flower bud), } \\
\text { taperera }\end{array}$ & $\mathrm{Bl}$ & $\begin{array}{l}\text { Floral bud. } \\
\text { Whole plant. }\end{array}$ & $\begin{array}{l}\text { Folk oral literature } \\
\text { Other informations: ecological information }\end{array}$ & 5 \\
\hline $\begin{array}{l}\text { Capsella bursa-pastoris (L.) } \\
\text { Medik. (Brassicaceae) } \\
\text { BCN46079 }\end{array}$ & Pare i fill & $\mathrm{Bl}$ & Not reported & Other informations & 1 \\
\hline $\begin{array}{l}\text { Capsicum annuum L. } \\
\text { (Solanaceae) BCN24737 }\end{array}$ & $\begin{array}{l}\text { Pebre covent, pebre covent } \\
\text { (fruit), pebre, pebrera, } \\
\text { nyora, pebre vermell, } \\
\text { pebrer, pebre de cirereta, } \\
\text { pebresser, cirereta picant } \\
\text { (fruit), pebre de banyeta } \\
\text { (fruit), pebre (fruit) }\end{array}$ & $\mathrm{Bl}$ & $\begin{array}{l}\text { Fruit. Whole } \\
\text { plant }\end{array}$ & $\begin{array}{l}\text { Agrosilvopastoral management: agricultural/ } \\
\text { horticultural } \\
\text { Magic and religious beliefs and practices } \\
\text { Ornamental: bouquet elaboration } \\
\text { Other informations: ecological information } \\
\text { Repellent }\end{array}$ & 14 \\
\hline Capsicum sp. (Solanaceae) & Pebre bord & $\mathrm{Bl}$ & Seed & $\begin{array}{l}\text { Agrosilvopastoral management: for pig } \\
\text { slaughter }\end{array}$ & 1 \\
\hline $\begin{array}{l}\text { Carlina acanthifolia All. } \\
\text { (Asteraceae) BCN24738 }\end{array}$ & Carlina, carolina & $\mathrm{Rl}$ & $\begin{array}{l}\text { Aerial part. } \\
\text { Whole plant }\end{array}$ & $\begin{array}{l}\text { Agrosilvopastoral management: agricultural/ } \\
\text { horticultural } \\
\text { Magic and religious beliefs and practices } \\
\text { Ornamental } \\
\text { Ornamental: bouquet elaboration } \\
\text { Other informations: harvesting and/or selling } \\
\text { Other informations: ecological information }\end{array}$ & 14 \\
\hline $\begin{array}{l}\text { Carlina acanthifolia All. } \\
\text { subsp. cynara (Pourr. ex } \\
\text { DC.) Rouy (Asteraceae) } \\
\text { BCN46095 }\end{array}$ & $\begin{array}{l}\text { Carlina, carlina angèlica, } \\
\text { carlinassa, carolina }\end{array}$ & $A E, M O$ & $\begin{array}{l}\text { Aerial part. } \\
\text { Whole plant }\end{array}$ & $\begin{array}{l}\text { Magic and religious beliefs and practices } \\
\text { Other informations: ecological information }\end{array}$ & 11 \\
\hline $\begin{array}{l}\text { Carlina acaulis L. } \\
\text { (Asteraceae) BCN32946 }\end{array}$ & Carlina & $\mathrm{AE}$ & Whole plant & Other informations: ecological information & 2 \\
\hline
\end{tabular}


Table 1 Non-food and non-medicinal folk plant uses in the territories of Alt Empordà, Montseny and Ripollès (Catalonia), and Formentera and Mallorca (Balearic Islands) (Continued)

Castanea sativa Mill. (Fagaceae) BCN29844
Castanya (fruit), castanyer

$A E, B I$,

$\mathrm{MO}, \mathrm{RI}$

Leaf.

Stem
Inflorescence.

Agrosilvopastoral management: for fishing

Agrosilvopastoral management: tools for

agricultural practices

Artisanal: basketry

Artisanal: cane elaboration

Domestic: for help at home

Ludic

Ornamental: gardening

Other informations: use not remembered

Timber: boat manufacturing

Timber: furniture manufacturing

Timber: construction materials production
Catananche caerulea $\mathrm{L}$. (Asteraceae) BCN29645

Manetti ex Carrière

(Pinaceae) BCN35722

Cedrus libani A.Rich.

(Pinaceae) BCN29978
Celtis australis L.

(Cannabaceae) BCN29845

$\begin{array}{lll}\begin{array}{l}\text { Cigales, pell de serp, pinya } \\ \text { de plata } \\ \text { Cedre }\end{array} & \mathrm{AE}, \mathrm{RI} & \begin{array}{l}\text { Aerial part. } \\ \text { Inflorescence }\end{array} \\ & \mathrm{MO} & \text { Whole plant } \\ \text { Cedre } & \mathrm{AE} & \text { Stem } \\ \begin{array}{l}\text { Lledoner, lledroner, lleroner, } \\ \text { lledó (fruit), lledró (fruit), } \\ \text { lleró (fruit) }\end{array} & & \begin{array}{l}\text { Stem. Whole } \\ \text { plant }\end{array}\end{array}$

Ludic

Ornamental: bouquet elaboration

Ornamental: gardening

Timber: furniture manufacturing

Tools for agricultural practices

Artisanal: broom elaboration

Artisanal: cane elaboration
Cedrus atlantica (Endl.)

Artisanal: musical instrument elaboration

Ludic

Magic and religious beliefs and practices

Ornamental: gardening

Other informations: ecological information

Other informations: use not remembered

Timber

Timber: boat manufacturing

Centaurea aspera L.

(Asteraceae) BCN42691

Bracera, herba de bracera

$\mathrm{BI}$

Aerial part.

Whole plant

Centaurea cyanus L.

(Asteraceae) BCN29651

Centaurea pectinata L.

subsp. pectinata

(Asteraceae) BCN43498

Centranthus ruber (L.) DC

subsp. ruber (Caprifoliaceae)

BCN31267

Ceratonia siliqua $\mathrm{L}$.

(Fabaceae) BCN32177

Garrofer, garrover, garrofa (fruit)

Blauet, blau, caps blaus, clavell de blat, clavell de Sant Isidre, llums

Barbassa, travalera, travarada

Rosa d'Espanya

AE, MO, RI Aerial part. Inflorescence

Agrosilvopastoral management: tools for agricultural practices

Other informations: ecological information

Magic and religious beliefs and practices Ornamental: bouquet elaboration

MO Aerial part Ludic

Ornamental: bouquet elaboration

Agrosilvopastoral management: honey

Artisanal: footwear elaboration

Cosmetic

Folk oral literature

Fuel obtaining: firewood

Other informations: harvesting and/or selling

Other informations: ecological information

Other informations: use not remembered

Textile: dyer

Timber: furniture manufacturing

Cercis siliquastrum L.

(Fabaceae) BCN51020

Árbol de la pasión

BI

Whole plant

Ornamental: gardening

(Spanish)

Auradella

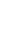


Table 1 Non-food and non-medicinal folk plant uses in the territories of Alt Empordà, Montseny and Ripollès (Catalonia), and Formentera and Mallorca (Balearic Islands) (Continued)

Chamaerops humilis L. (Arecaceae) BCN23832

\begin{abstract}
Bri (leaflet), clin, garballó, garbeó, Ilatra (elaborated product), margalló, palma, palmito (fruit) (Spanish), pauma, paumera (group of palms), paumissó (fruit), pomera, pomissó (fruit), punissó (fruit)
\end{abstract}

Chenopodium ambrosioides
L. (Amaranthaceae)
BCN103590
Chlorophytum comosum
(Thunb.) Jacques
(Asparagaceae) BC617113
Chrysanthemum coronarium
L. (Asteraceae) BCN95559
Chrysanthemum frutescens
L. (Asteraceae) BCN93699
Chrysanthemum segetum L.
(Asteraceae) BCN31269
Chrysanthemum sinense
Sabine (Asteraceae)
BCN129085
Cicer arietinum L.
(Fabaceae) BCN29659
Cichorium endivia L.
(Asteraceae) BCN46854
Cichorium intybus L.
(Asteraceae) BCN29660
Cistus albidus L. (Cistaceae)
CCN36672
(L.) J.Presl (Lauraceae)
BCN50766
(Assium arvense (L.) Scop.
(Asteraceae) BCN29853 vulgare (Savi) Ten.
(Anamom BCN E-63
(A)

Te mallorquí

Cossiol de cintes

Bòlig, sardonaia

Margalida

Goit

Estranys

Ciuró (seed)

Endívia

Cama-rotja, cama-roja, masteguera

Càmfora (elaborated product)

Calcida

Card

Estepa, estepa blanca, estepa d'escurar, estepa rosa

Estepa blanca, estepa borda, estepa de fulla llarga, estepa negra, mòdega

Cistus salviifolius L. (Cistaceae) BCN36767

Citrullus lanatus (Thunb.) Matsum. \& Nakai (Cucurbitaceae) BCN29662

Estepa, estepa negra, estepa bona, estepa borrera, esteperola,

Síndria (fruit), sindriera

$\begin{array}{ll}\mathrm{AE}, \mathrm{BI} & \text { Leaf. Leaflet. } \\ & \text { Not reported. } \\ & \text { Stem. Whole } \\ & \text { plant }\end{array}$

Artisanal: basketry

Artisanal: broom elaboration

Domestic: for help at home

Folk oral literature

Magic and religious beliefs and practices

Ornamental: bouquet elaboration

Ornamental: gardening

Other informations: ecological information

Textile: fibre or cloth elaboration

Textile: textile padding

$\mathrm{Bl} \quad$ Whole plant

Other informations: appreciations on plant or product properties

Bl Whole plant

Ornamental: gardening

BI Inflorescence Ludic

BI Whole plant

Ornamental: gardening

AE $\quad$ Whole plant

Other informations: ecological information

BI Whole plant

Magic and religious beliefs and practices Repellent

Agrosilvopastoral management: agricultural/ horticultural

$\mathrm{Bl} \quad$ Leaf

Other informations: ecological information

$\mathrm{AE}, \mathrm{BI} \quad$ Leaf. Whole

Agrosilvopastoral management: agricultural/

horticultural

Folk oral literature

Other informations: ecological information

$\mathrm{AE}, \mathrm{BI} \quad$ Bark. Not $\quad$ Magic and religious beliefs and practices

Other informations: purchased in commerce Repellent

$\mathrm{RI}, \mathrm{AE} \quad$ Whole plant Other informations: ecological information

MO Inflorescence Magic and religious beliefs and practices

$\mathrm{AE}, \mathrm{BI}, \mathrm{MO}$ Aerial part.

Agrosilvopastoral management: for pig

Flower. Not slaughter

reported. Stem. Domestic: for help at home

Whole plant Fuel obtaining: firewood

Ornamental: bouquet elaboration

Other informations: ecological information

$\mathrm{AE}, \mathrm{MO} \quad$ Flower. Resin. Agrosilvopastoral management: tools for Stem. Whole agricultural practices

plant.

Artisanal: toy elaboration

Domestic: for help at home

Other informations: ecological information
$\mathrm{AE}, \mathrm{BI}, \mathrm{MO}$ Flower. Leaf. Stem. Whole plant

Agrosilvopastoral management: agricultural/ horticultural

Domestic: for help at home

Other informations: ecological information Smoking $\mathrm{BI}$

Fruit
Magic and religious beliefs and practices Folk oral literature
49

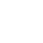


Table 1 Non-food and non-medicinal folk plant uses in the territories of Alt Empordà, Montseny and Ripollès (Catalonia), and Formentera and Mallorca (Balearic Islands) (Continued)

Citrus xaurantium L.
(Rutaceae) BCN46080
Citrus grandis (L.) Osbeck
(Rutaceae) BCN52090
Citrus limon (L.) Osbeck
(Rutaceae) BCN46853

(Rutaceae) BCN46853
(Rutaceae) BCN-E250

Citrus sinensis (L.) Osbeck (Rutaceae) BCN24752

Taronja agra (fruit)
Pomelo (fruit)
Bergamota (fruit), llimona
(fruit), llimoner, llimonera,
mandarina (fruit), taronger
bord, taronger clementí,
taronger mandarí, taronja
borda (fruit), taronja de la
seca (fruit), taronja de pelar
grillons (fruit), taronja de
navel (fruit), pomelo (fruit)

Pomelo (fruit)

Taronger, taronja (fruit)

A

\begin{tabular}{|c|c|}
\hline $\mathrm{AE}$ & Fruit \\
\hline$A E$ & Whole plant \\
\hline $\mathrm{AE}, \mathrm{BI}$ & $\begin{array}{l}\text { Epicarp. Fruit. } \\
\text { Fruit juice. } \\
\text { Whole plant }\end{array}$ \\
\hline $\mathrm{Bl}$ & Fruit \\
\hline $\mathrm{BI}$ & $\begin{array}{l}\text { Flower. Fruit. } \\
\text { Leaf. Stem }\end{array}$ \\
\hline
\end{tabular}

Agrosilvopastoral management: for pig

8 slaughter

Other informations: ecological information

1

Agrosilvopastoral management: for pig

slaughter

Cosmetic

Domestic: air freshener

Domestic: for help at home

Folk oral literature

Other informations: ecological information

Repellent

Other informations: use not remembered

Agrosilvopastoral management: for pig

slaughter

Artisanal: cane elaboration

Artisanal: cookware elaboration

Folk oral literature

Ludic

Magic and religious beliefs and practices

Other informations: undesirable actions

Timber

\begin{abstract}
Cladonia sp. (Cladoniaceae)
Clematis cirrhosa L.

(Ranunculaceae) BCN22034

Clematis flammula L. (Ranunculaceae) BCN29856

Herba de la roca, herba roquera, musgo (Spanish)

Vidauba, villauba

Didorta, joanillo, ridolta, ridorta, viadella, vidriella, vidauba
\end{abstract}

Clematis vitalba $\mathrm{L}$.

(Ranunculaceae) BCN29857
$\mathrm{BI}$

BI

$A E, B I, M O$ Aerial part. Flower. Stem. Whole plan

$A E, M O, R I$ Not reported. Stem. Whole plant

(Rutaceae) BCN95567

Coffea arabica L.

(Rubiaceae) BCN-E-65

Coix lacryma-jobi L. (Poaceae) BC917450

Colchicum autumnale L. subsp. autumnale (Liliaceae) BCN45283

Colocasia sp. (Araceae)

Colutea arborescens L. subsp. gallica Browicz (Fabaceae) BCN56002

Conium maculatum L. (Apiaceae) BCN32171

Consolida ajacis (L.) Schur (Ranunculaceae) BCN60924

Convallaria majalis L. (Liliaceae) BCN57362
Didorta, llidorta, ridolta, ridorta, vidiella

\section{Raspall}

$\mathrm{BI}$

Cafè (seed)
Llàgrimes de Job, Ilàgrimes
de viu
Cabeça

Colocàsia

Pleps

$\mathrm{MO}$

Aerial part. Not reported

seed

Fruit

Tuber

Whole plant

Stem

Whole plant

Folk oral literature

Flower. Whole plant

Whole plant

Folk oral literature

Folk oral literature

Folk oral literature

\section{Cicuta}

Espuela (Spanish)
Domestic: for help at home

Magic and religious beliefs and practices

Ornamental: bouquet elaboration

Ornamental: gardening

Other informations: use not remembered

Agrosilvopastoral management: tools for agricultural practices

Domestic: for help at home

Ornamental: bouquet elaboration

Other informations: ecological information

Agrosilvopastoral management: tools for agricultural practices

Ludic

Other informations: ecological information Smoking

Artisanal: broom elaboration

Magic and religious beliefs and practices

Magic and religious beliefs and practices

Ornamental: bouquet elaboration
Magic and religious beliefs and practices Ornamental: gardening

Ornamental: gardening
1 
Table 1 Non-food and non-medicinal folk plant uses in the territories of Alt Empordà, Montseny and Ripollès (Catalonia), and Formentera and Mallorca (Balearic Islands) (Continued)

\begin{tabular}{|c|c|c|c|c|c|}
\hline $\begin{array}{l}\text { Convolvulus arvensis L. } \\
\text { (Convolvulaceae) } \\
\text { BCN109798 }\end{array}$ & $\begin{array}{l}\text { Correjola, corretjola, } \\
\text { corriola, corritjola }\end{array}$ & $\mathrm{BI}, \mathrm{MO}$ & $\begin{array}{l}\text { Flower. Whole } \\
\text { plant }\end{array}$ & $\begin{array}{l}\text { Agrosilvopastoral management: weeds } \\
\text { Ludic } \\
\text { Other informations: excipient or adjuvant in } \\
\text { medicinal or food preparations }\end{array}$ & 3 \\
\hline $\begin{array}{l}\text { Coriaria myrtifolia L. } \\
\text { (Coriariaceae) BCN24754 }\end{array}$ & Rodó, roldor & $\mathrm{Rl}$ & Leaf & Magic and religious beliefs and practices & 1 \\
\hline $\begin{array}{l}\text { Coris monspeliensis L. subsp. } \\
\text { monspeliensis (Primulaceae) } \\
\text { BCN110450 }\end{array}$ & $\begin{array}{l}\text { Farigola mascle, farigola de } \\
\text { pastor, tomanyí }\end{array}$ & $\mathrm{MO}$ & Flower & $\begin{array}{l}\text { Agrosilvopastoral management: honey } \\
\text { obtaining }\end{array}$ & 1 \\
\hline $\begin{array}{l}\text { Cornus sanguinea L. } \\
\text { (Cornaceae) BCN29979 }\end{array}$ & Sanguinyol & $\mathrm{AE}$ & Sap & Magic and religious beliefs and practices & 1 \\
\hline $\begin{array}{l}\text { Coronilla scorpioides K.Koch } \\
\text { (Fabaceae) BCN22206 }\end{array}$ & Herba enamorada & $\mathrm{Bl}$ & Whole plant & Folk oral literature & 1 \\
\hline $\begin{array}{l}\text { Cortaderia selloana (Schult. } \\
\text { \& Schult.f.) Asch. \& Graebn. } \\
\text { (Poaceae) BCN81361 }\end{array}$ & Plomalls & $\mathrm{MO}$ & Aerial part & Ornamental: bouquet elaboration & 1 \\
\hline $\begin{array}{l}\text { Corylus avellana L. } \\
\text { (Betulaceae) BCN29831 }\end{array}$ & $\begin{array}{l}\text { Avellana (fruit), avellaner, } \\
\text { avellaner bord }\end{array}$ & $\mathrm{AE}, \mathrm{BI}, \mathrm{MO}$ & Leaf. Stem & $\begin{array}{l}\text { Agrosilvopastoral management: tools for } \\
\text { agricultural practices } \\
\text { Artisanal: cane elaboration } \\
\text { Magic and religious beliefs and practices } \\
\text { Smoking } \\
\text { Timber: construction materials production }\end{array}$ & 12 \\
\hline $\begin{array}{l}\text { Crataegus azarolus L. } \\
\text { (Rosaceae) BCN14977 }\end{array}$ & Atzerola (fruit), atzerolera & $\mathrm{Bl}$ & $\begin{array}{l}\text { Stem. Whole } \\
\text { plant }\end{array}$ & $\begin{array}{l}\text { Agrosilvopastoral management: tools for } \\
\text { agricultural practices } \\
\text { Timber }\end{array}$ & 2 \\
\hline $\begin{array}{l}\text { Crataegus monogyna Jacq. } \\
\text { (Rosaceae) BCN103563 }\end{array}$ & $\begin{array}{l}\text { Cirerer de pastor, cireretes } \\
\text { de pastor (fruit), espinal, } \\
\text { espinaler, garganyer }\end{array}$ & $\mathrm{Bl}$ & Whole plant & $\begin{array}{l}\text { Agrosilvopastoral management: agricultural/ } \\
\text { horticultural } \\
\text { Other informations: ecological information }\end{array}$ & 5 \\
\hline $\begin{array}{l}\text { Crataegus monogyna Jacq. } \\
\text { subsp. monogyna } \\
\text { (Rosaceae) BCN29858 }\end{array}$ & $\begin{array}{l}\text { Arç, arç blanc, arn, arn } \\
\text { blanc }\end{array}$ & $\mathrm{AE}, \mathrm{MO}, \mathrm{Rl}$ & $\begin{array}{l}\text { Aerial part. } \\
\text { Flower. Whole } \\
\text { plant }\end{array}$ & $\begin{array}{l}\text { Agrosilvopastoral management: tools for } \\
\text { agricultural practices } \\
\text { Folk oral literature } \\
\text { Ornamental: bouquet elaboration }\end{array}$ & 9 \\
\hline $\begin{array}{l}\text { Crithmum maritimum L. } \\
\text { (Apiaceae) BCN104272 }\end{array}$ & Fonoll marí & $\mathrm{Bl}$ & Aerial part & $\begin{array}{l}\text { Folk oral literature } \\
\text { Other informations: ecological information }\end{array}$ & 2 \\
\hline $\begin{array}{l}\text { Crocus sativus L. (Iridaceae) } \\
\text { BCN32170 }\end{array}$ & Safà & $\mathrm{Bl}$ & Perigonium & Folk oral literature & 1 \\
\hline $\begin{array}{l}\text { Cucumis melo L. } \\
\text { (Cucurbitaceae) BCN46851 }\end{array}$ & $\begin{array}{l}\text { Meló (fruit), moló (fruit), } \\
\text { melonera, molonera, }\end{array}$ & $\mathrm{Bl}$ & $\begin{array}{l}\text { Epicarp. Fruit. } \\
\text { Whole plant }\end{array}$ & $\begin{array}{l}\text { Folk oral literature } \\
\text { Ludic } \\
\text { Magic and religious beliefs and practices } \\
\text { Other informations: ecological information }\end{array}$ & 5 \\
\hline $\begin{array}{l}\text { Cucumis sativus L. } \\
\text { (Cucurbitaceae) BCN46850 }\end{array}$ & $\begin{array}{l}\text { Cogombre, pepino } \\
\text { (Spanish) }\end{array}$ & $\mathrm{Bl}, \mathrm{MO}$ & $\begin{array}{l}\text { Fruit. Whole } \\
\text { plant }\end{array}$ & $\begin{array}{l}\text { Agrosilvopastoral management: agricultural/ } \\
\text { horticultural } \\
\text { Cosmetic }\end{array}$ & 2 \\
\hline $\begin{array}{l}\text { Cucurbita pepo L. } \\
\text { (Cucurbitaceae) BCN49858 }\end{array}$ & Carabassera & $\mathrm{Bl}$ & $\begin{array}{l}\text { Flower. Fruit. } \\
\text { Whole plant }\end{array}$ & $\begin{array}{l}\text { Agrosilvopastoral management: agricultural/ } \\
\text { horticultural } \\
\text { Folk oral literature } \\
\text { Magic and religious beliefs and practices }\end{array}$ & 4 \\
\hline $\begin{array}{l}\text { Cucurbita pepo L. var. } \\
\text { oblonga (Cucurbitaceae) } \\
\text { BCN29859 }\end{array}$ & Carabassó (fruit) & $\mathrm{AE}$ & Fruit & Ludic & 2 \\
\hline $\begin{array}{l}\text { Cupressus macrocarpa } \\
\text { Hartw. (Cupressaceae) } \\
\text { BCN35775 }\end{array}$ & Ciprer & $\mathrm{Bl}$ & Stem & Fuel obtaining: firewood & 1 \\
\hline $\begin{array}{l}\text { Cupressus sempervirens L. } \\
\text { (Cupressaceae) BCN95563 }\end{array}$ & $\begin{array}{l}\text { Xifrer, xifrer baix, xifrer de } \\
\text { fer tallavents, xiprer }\end{array}$ & $\mathrm{AE}, \mathrm{Bl}, \mathrm{RI}$ & $\begin{array}{l}\text { Fruictification. } \\
\text { Whole plant }\end{array}$ & $\begin{array}{l}\text { Agrosilvopastoral management: tools for } \\
\text { agricultural practices } \\
\text { Domestic: air freshener } \\
\text { Magic and religious beliefs and practices } \\
\text { Ornamental: gardening } \\
\text { Other informations: ecological information }\end{array}$ & 7 \\
\hline
\end{tabular}


Table 1 Non-food and non-medicinal folk plant uses in the territories of Alt Empordà, Montseny and Ripollès (Catalonia), and Formentera and Mallorca (Balearic Islands) (Continued)

\begin{tabular}{|c|c|c|c|c|c|}
\hline $\begin{array}{l}\text { Cuscuta epithymum (L.) L. } \\
\text { (Convolvulaceae) } \\
\text { BCN112569 }\end{array}$ & Tinya & $\mathrm{Rl}$ & Whole plant & Folk oral literature & 1 \\
\hline $\begin{array}{l}\text { Cydonia oblonga Mill. } \\
\text { (Rosaceae) BCN46849 }\end{array}$ & Codony (fruit), codonyer & $\begin{array}{l}\mathrm{AE}, \mathrm{BI} \\
\mathrm{MO}, \mathrm{RI}\end{array}$ & Fruit & $\begin{array}{l}\text { Domestic: air freshener } \\
\text { Other informations: ecological information }\end{array}$ & 4 \\
\hline $\begin{array}{l}\text { Cynara cardunculus L. } \\
\text { (Asteraceae) BCN56003 }\end{array}$ & $\begin{array}{l}\text { Card gros, carxofera, card } \\
\text { coler, card calapoter, } \\
\text { carxofera borda, herba-col, } \\
\text { presonera, presora }\end{array}$ & $\mathrm{BI}, \mathrm{MO}$ & $\begin{array}{l}\text { Aerial part. } \\
\text { Whole plant }\end{array}$ & $\begin{array}{l}\text { Folk oral literature } \\
\text { Ornamental: bouquet elaboration }\end{array}$ & 4 \\
\hline $\begin{array}{l}\text { Cynara scolymus L. } \\
\text { (Asteraceae) BCN24760 }\end{array}$ & $\begin{array}{l}\text { Carxofa (inflorescence), } \\
\text { carxofera }\end{array}$ & $\mathrm{Bl}$ & $\begin{array}{l}\text { Leaf. Whole } \\
\text { plant }\end{array}$ & $\begin{array}{l}\text { Agrosilvopastoral management: tool for } \\
\text { agricultural practices } \\
\text { Other informations: ecological information }\end{array}$ & 2 \\
\hline $\begin{array}{l}\text { Cynodon dactylon (L.) Pers. } \\
\text { (Poaceae) BCN24760 }\end{array}$ & Gram, grama, xerpolla & $\mathrm{Bl}, \mathrm{MO}, \mathrm{Rl}$ & $\begin{array}{l}\text { Aerial part. } \\
\text { Whole plant }\end{array}$ & $\begin{array}{l}\text { Agrosilvopastoral management: weeds } \\
\text { Folk oral literature } \\
\text { Magic and religious beliefs and practices } \\
\text { Ornamental: gardening }\end{array}$ & 5 \\
\hline $\begin{array}{l}\text { Cynoglossum creticum Mill. } \\
\text { (Boraginaceae) BCN97779 }\end{array}$ & $\begin{array}{l}\text { Llapassa, llapassera, } \\
\text { llapissera }\end{array}$ & $\mathrm{Bl}$ & $\begin{array}{l}\text { Fruit. Leaf. } \\
\text { Seed. Whole } \\
\text { plant }\end{array}$ & $\begin{array}{l}\text { Folk oral literature } \\
\text { Other informations: ecological information } \\
\text { Smoking }\end{array}$ & 4 \\
\hline $\begin{array}{l}\text { Cyperus alternifolius L. } \\
\text { subsp. flabelliformis (Rottb.) } \\
\text { M.R.Almeida (Cyperaceae) } \\
\text { BCN } 16451\end{array}$ & Papiro (Spanish) & $\mathrm{Bl}$ & Stem & Other informations: ecological information & 1 \\
\hline $\begin{array}{l}\text { Cyperus eragrostis Lam. } \\
\text { (Cyperaceae) BCN61182 }\end{array}$ & $\begin{array}{l}\text { Castanyola, jonc, paraigüet, } \\
\text { serrana }\end{array}$ & $\mathrm{MO}$ & Aerial part & $\begin{array}{l}\text { Agrosilvopastoral management: tools for } \\
\text { agricultural practices } \\
\text { Ornamental: bouquet elaboration }\end{array}$ & 3 \\
\hline $\begin{array}{l}\text { Cyperus rotundus L. } \\
\text { (Cyperaceae) BCN39997 }\end{array}$ & Jonc & $\mathrm{AE}$ & Stem & $\begin{array}{l}\text { Agrosilvopastoral management: tools for } \\
\text { agricultural practices } \\
\text { Artisanal: basketry }\end{array}$ & 2 \\
\hline $\begin{array}{l}\text { Cystoseira amentacea Bory } \\
\text { var. stricta Mont. } \\
\text { (Cystoseiraceae) BCN-Phyc- } \\
6232\end{array}$ & Herba saupera & $\mathrm{Bl}$ & Frond & Agrosilvopastoral management: for fishing & 1 \\
\hline $\begin{array}{l}\text { Cystoseira crinita Duby } \\
\text { (Cystoseiraceae) BCN-Phyc- } \\
6233\end{array}$ & Herba corretgera & $\mathrm{Bl}$ & Frond & Agrosilvopastoral management: for fishing & 1 \\
\hline $\begin{array}{l}\text { Cystoseira elegans Sauv. } \\
\text { (Cystoseiraceae) BCN-Phyc- } \\
6150\end{array}$ & Herba borda & $\mathrm{Bl}$ & Frond & Agrosilvopastoral management: for fishing & 1 \\
\hline $\begin{array}{l}\text { Cytinus hypocistis (L.) L. } \\
\text { subsp. macranthus Wettst. } \\
\text { (Rafflesiaceae) BCN2224 }\end{array}$ & $\begin{array}{l}\text { Magraneta, magraneta } \\
\text { d'esteperol }\end{array}$ & $\mathrm{Bl}$ & Not reported & Textile: dyer & 4 \\
\hline $\begin{array}{l}\text { Dalbergia sp. (Fabaceae) } \\
\text { BCN52091 }\end{array}$ & Palissandre & $A E$ & Stem & Artisanal: musical instrument elaboration & 1 \\
\hline Dalhia sp. (Asteraceae) & Dàlia, daliera & $\mathrm{Bl}$ & Whole plant & $\begin{array}{l}\text { Agrosilvopastoral management: tools for } \\
\text { agricultural practices } \\
\text { Ornamental: gardening }\end{array}$ & 4 \\
\hline $\begin{array}{l}\text { Daphne gnidium L. } \\
\text { (Thymelaeaceae) BCN29687 }\end{array}$ & Matapoll, tei, tintorell & $\mathrm{AE}, \mathrm{BI}, \mathrm{MO}$ & $\begin{array}{l}\text { Bark. Root. } \\
\text { Stem }\end{array}$ & $\begin{array}{l}\text { Agrosilvopastoral management: for fishing } \\
\text { Artisanal: basketry } \\
\text { Artisanal: broom elaboration } \\
\text { Domestic: for help at home } \\
\text { Folk oral literature }\end{array}$ & 9 \\
\hline $\begin{array}{l}\text { Datura stramonium L. } \\
\text { (Solanaceae) BCN29688 }\end{array}$ & $\begin{array}{l}\text { Castanyer bord, datura } \\
\text { borda, estramoni, flor de } \\
\text { mort, herba de capseta, } \\
\text { herba talpera, herba } \\
\text { taupera, matataups, } \\
\text { trompetera }\end{array}$ & $\begin{array}{l}\mathrm{AE}, \mathrm{BI} \\
\mathrm{MO}, \mathrm{RI}\end{array}$ & $\begin{array}{l}\text { Aerial part. } \\
\text { Leaf. Flower. } \\
\text { Whole plant }\end{array}$ & $\begin{array}{l}\text { Agrosilvopastoral management: agricultural/ } \\
\text { horticultural } \\
\text { Magic and religious beliefs and practices } \\
\text { Ornamental: gardening } \\
\text { Other informations: ecological information } \\
\text { Repellent }\end{array}$ & 12 \\
\hline
\end{tabular}


Table 1 Non-food and non-medicinal folk plant uses in the territories of Alt Empordà, Montseny and Ripollès (Catalonia), and Formentera and Mallorca (Balearic Islands) (Continued)

\begin{tabular}{|c|c|c|c|c|c|}
\hline $\begin{array}{l}\text { Daucus carota L. subsp. } \\
\text { carota (Apiaceae) } \\
\text { BCN48714 }\end{array}$ & $\begin{array}{l}\text { Botxa, caps blancs, carrota } \\
\text { borda, fonollassa, julivert } \\
\text { bord, julivert de galipau, } \\
\text { moixos (inflorescence), } \\
\text { pastanaga borda, rosella } \\
\text { borda }\end{array}$ & $\mathrm{AE}, \mathrm{BI}, \mathrm{MO}$ & $\begin{array}{l}\text { Aerial part. } \\
\text { Inflorescence. } \\
\text { Not reported }\end{array}$ & $\begin{array}{l}\text { Agrosilvopastoral management: for pig } \\
\text { slaughter } \\
\text { Magic and religious beliefs and practices } \\
\text { Ornamental: bouquet elaboration } \\
\text { Other informations: ecological information }\end{array}$ & 5 \\
\hline $\begin{array}{l}\text { Delphinium ajacis L. } \\
\text { (Ranunculaceae) BCN31271 }\end{array}$ & Espuela (Spanish) & $\mathrm{AE}$ & Aerial part & Ornamental: bouquet elaboration & 1 \\
\hline $\begin{array}{l}\text { Dianthus caryophyllus L. } \\
\text { (Caryophyllaceae) } \\
\text { BCN31272 }\end{array}$ & $\begin{array}{l}\text { Clavell, clavell blanc, clavell } \\
\text { d'hivernacle }\end{array}$ & $\mathrm{AE}, \mathrm{MO}, \mathrm{Rl}$ & $\begin{array}{l}\text { Aerial part. } \\
\text { Flower }\end{array}$ & $\begin{array}{l}\text { Magic and religious beliefs and practices } \\
\text { Ornamental: bouquet elaboration }\end{array}$ & 12 \\
\hline $\begin{array}{l}\text { Dianthus hyssopifolius L. } \\
\text { (Caryophyllaceae) } \\
\text { BCN27299 }\end{array}$ & Clavell & $\mathrm{Rl}$ & Flower & Magic and religious beliefs and practices & 1 \\
\hline $\begin{array}{l}\text { Dianthus seguieri Vill. } \\
\text { (Caryophyllaceae) } \\
\text { BCN24763 }\end{array}$ & Clavell de muntanya & $\mathrm{Rl}$ & Flower & Magic and religious beliefs and practices & 1 \\
\hline $\begin{array}{l}\text { Dianthus seguieri Vill. subsp. } \\
\text { requienii (Godr.) M.Bernal, } \\
\text { Laínz \& Muñoz Garm. } \\
\text { (Caryophyllaceae) } \\
\text { BCN22276 }\end{array}$ & $\begin{array}{l}\text { Clavell de bosc, clavellde } \\
\text { pastor_ }\end{array}$ & $A E$ & Whole plant & Ornamental: bouquet elaboration & 1 \\
\hline $\begin{array}{l}\text { Dianthus sp. } \\
\text { (Caryophyllaceae) }\end{array}$ & $\begin{array}{l}\text { Clavell, clavell de Sant } \\
\text { Jaume }\end{array}$ & $\mathrm{Bl}, \mathrm{Rl}$ & $\begin{array}{l}\text { Aerial part. } \\
\text { Flower }\end{array}$ & $\begin{array}{l}\text { Folk oral literature } \\
\text { Ornamental: bouquet elaboration }\end{array}$ & 3 \\
\hline $\begin{array}{l}\text { Dianthus pungens L. subsp. } \\
\text { pungens (Caryophyllaceae) } \\
\text { BCN46087 }\end{array}$ & Coixinet de monja & $\mathrm{AE}$ & Whole plant & Other informations: ecological information & 1 \\
\hline $\begin{array}{l}\text { Diospyros ebenum J.Koenig } \\
\text { ex Retz. (Ebenaceae) } \\
\text { BCN52092 }\end{array}$ & Banús & $\mathrm{AE}$ & Stem & Artisanal: musical instrument elaboration & 1 \\
\hline $\begin{array}{l}\text { Diospyros kaki L.f. } \\
\text { (Ebenaceae) BCN52627 }\end{array}$ & $\begin{array}{l}\text { Caqui (fruit), caquier, } \\
\text { clequier }\end{array}$ & $\mathrm{Bl}$ & Fruit. Stem & $\begin{array}{l}\text { Agrosilvopastoral management: agricultural/ } \\
\text { horticultural } \\
\text { Artisanal: musical instrument elaboration } \\
\text { Other informations: excipient or adjuvant in } \\
\text { medicinal or food preparations }\end{array}$ & 3 \\
\hline $\begin{array}{l}\text { Diplotaxis erucoides (L.) DC. } \\
\text { (Brassicaceae) BCN29861 }\end{array}$ & Cap blanc, ravenissa & $\mathrm{AE}$ & Whole plant & Other informations: ecological information & 5 \\
\hline $\begin{array}{l}\text { Dipsacus fullonum L. } \\
\text { (Caprifoliaceae) BCN27266 }\end{array}$ & $\begin{array}{l}\text { Assotacristos, card, cardassa, } \\
\text { cristos, escardot, } \\
\text { escarpidora, escarpido, } \\
\text { escarpín }\end{array}$ & $\mathrm{Bl}, \mathrm{MO}, \mathrm{Rl}$ & $\begin{array}{l}\text { Aerial part. } \\
\text { Flower. } \\
\text { Inflorescence }\end{array}$ & $\begin{array}{l}\text { Domestic: for help at home } \\
\text { Ornamental: bouquet elaboration }\end{array}$ & 10 \\
\hline $\begin{array}{l}\text { Dipsacus fullonum L. subsp. } \\
\text { fullonum (Caprifoliaceae) } \\
\text { BCN29691 }\end{array}$ & Cardó, cap de burro & $\mathrm{AE}$ & Aerial part & Ornamental: bouquet elaboration & 3 \\
\hline $\begin{array}{l}\text { Dorycnium pentaphyllum } \\
\text { Scop. (Fabaceae) } \\
\text { BCN103561 }\end{array}$ & $\begin{array}{l}\text { Botxa de cuques, socarrell, } \\
\text { tijarell }\end{array}$ & $\mathrm{Bl}$ & Aerial part. Leaf & $\begin{array}{l}\text { Artisanal: broom elaboration } \\
\text { Fuel obtaining: firewood }\end{array}$ & 2 \\
\hline $\begin{array}{l}\text { Dorycnium pentaphyllum } \\
\text { Scop. subsp. pentaphyllum } \\
\text { (Fabaceae) BCN115631 }\end{array}$ & Botxa & $\mathrm{MO}$ & Flower & $\begin{array}{l}\text { Agrosilvopastoral management: agricultural/ } \\
\text { horticultural }\end{array}$ & 1 \\
\hline $\begin{array}{l}\text { Dryopteris filix-mas (L.) } \\
\text { Schott (Dryopteridaceae) } \\
\text { BCN29692 }\end{array}$ & Foguera, foguera de jardí & $A E, M O$ & $\begin{array}{l}\text { Frond. Whole } \\
\text { plant }\end{array}$ & Ornamental: bouquet elaboration & 3 \\
\hline $\begin{array}{l}\text { Ecballium elaterium (L.) } \\
\text { A.Rich. (Cucurbitaceae) } \\
\text { BCF44970 }\end{array}$ & $\begin{array}{l}\text { Cobrombo silvestre, } \\
\text { cogombre bord }\end{array}$ & $\mathrm{Bl}, \mathrm{MO}$ & $\begin{array}{l}\text { Fruit. Whole } \\
\text { plant }\end{array}$ & $\begin{array}{l}\text { Ludic } \\
\text { Other informations: ecological information }\end{array}$ & 2 \\
\hline $\begin{array}{l}\text { Echinocactus grusonii Hildm. } \\
\text { (Cactaceae) BCN129080 }\end{array}$ & Seient de sa sogra, grossini & $\mathrm{Bl}$ & Whole plant & Ornamental: gardening & 1 \\
\hline
\end{tabular}


Table 1 Non-food and non-medicinal folk plant uses in the territories of Alt Empordà, Montseny and Ripollès (Catalonia), and Formentera and Mallorca (Balearic Islands) (Continued)

\begin{tabular}{|c|c|c|c|c|c|}
\hline $\begin{array}{l}\text { Echinops ritro L. subsp. ritro } \\
\text { (Asteraceae) BCN31273 }\end{array}$ & Espines blaves & $A E$ & Aerial part & Ornamental: bouquet elaboration & 5 \\
\hline $\begin{array}{l}\text { Emex spinosa (L.) Campd. } \\
\text { (Polygonaceae) BCN13757 }\end{array}$ & Bledera borda, picatalons & $\mathrm{Bl}$ & Fruit & Other informations: ecological information & 1 \\
\hline $\begin{array}{l}\text { Epipremnum aureum } \\
\text { (Linden \& André) } \\
\text { G.S.Bunting (Araceae) } \\
\text { BCN129081 }\end{array}$ & Potos & $\mathrm{Bl}$ & Whole plant & Ornamental: gardening & 1 \\
\hline $\begin{array}{l}\text { Equisetum arvense L. } \\
\text { (Equisetaceae) BCN24767 }\end{array}$ & Cua de cavall, escuraplata & $\mathrm{Rl}$ & Aerial part & Domestic: for help at home & 1 \\
\hline $\begin{array}{l}\text { Equisetum ramosissimum } \\
\text { Desf. (Equisetaceae) } \\
\text { BCN29982 }\end{array}$ & $\begin{array}{l}\text { Coa de cavall, cua de cavall, } \\
\text { cua de rata }\end{array}$ & $A E, B I$ & $\begin{array}{l}\text { Aerial part. } \\
\text { Stem }\end{array}$ & $\begin{array}{l}\text { Agrosilvopastoral management: tools for } \\
\text { agricultural practices } \\
\text { Other informations: ecological information }\end{array}$ & 2 \\
\hline $\begin{array}{l}\text { Equisetum telmateia Ehrh. } \\
\text { (Equisetaceae) BCN118778 }\end{array}$ & $\begin{array}{l}\text { Coa de cavall, cova de } \\
\text { cavall, cua de cavall, } \\
\text { sangnua }\end{array}$ & $\mathrm{Bl}, \mathrm{MO}$ & $\begin{array}{l}\text { Aerial part. } \\
\text { Stem. Whole } \\
\text { plant }\end{array}$ & $\begin{array}{l}\text { Agrosilvopastoral management: tools for } \\
\text { agricultural practices } \\
\text { Cosmetic } \\
\text { Ludic } \\
\text { Magic and religious beliefs and practices } \\
\text { Other informations: ecological information }\end{array}$ & 5 \\
\hline $\begin{array}{l}\text { Erica arborea L. (Ericaceae) } \\
\text { BCN46081 }\end{array}$ & $\begin{array}{l}\text { Bruc, bruc bouer, bruc } \\
\text { femella, bruc de fer } \\
\text { escombres, bruc vertader, } \\
\text { mascle }\end{array}$ & $\mathrm{AE}, \mathrm{MO}, \mathrm{Rl}$ & $\begin{array}{l}\text { Aerial part. } \\
\text { Flower. Leaf. } \\
\text { Stem }\end{array}$ & $\begin{array}{l}\text { Agrosilvopastoral management: tools for } \\
\text { agricultural practices } \\
\text { Artisanal: broom elaboration } \\
\text { Artisanal: cookware elaboration } \\
\text { Artisanal: smoking pipe elaboration } \\
\text { Fuel obtaining: charcoal } \\
\text { Fuel obtaining: firewood } \\
\text { Ludic } \\
\text { Magic and religious beliefs and practices } \\
\text { Domestic: for help at home } \\
\text { Ornamental: bouquet elaboration } \\
\text { Other informations: ecological information }\end{array}$ & 58 \\
\hline $\begin{array}{l}\text { Erica multiflora L. (Ericaceae) } \\
\text { BCN29864 }\end{array}$ & $\begin{array}{l}\text { Bruc, bruc de bou, bruc de } \\
\text { llei, bruc mascle, cepell, } \\
\text { ciprell, ciprelló, petarrell, } \\
\text { xiprell }\end{array}$ & $\mathrm{AE}, \mathrm{BI}, \mathrm{MO}$ & $\begin{array}{l}\text { Aerial part. } \\
\text { Flower. Stem. } \\
\text { Whole plant }\end{array}$ & $\begin{array}{l}\text { Agrosilvopastoral management: for pig } \\
\text { slaughter } \\
\text { Artisanal: broom elaboration } \\
\text { Artisanal: smoking pipe elaboration } \\
\text { Fuel obtaining: charcoal } \\
\text { Fuel obtaining: firewood } \\
\text { Magic and religious beliefs and practices } \\
\text { Ornamental: bouquet elaboration } \\
\text { Ornamental: gardening } \\
\text { Other informations: ecological information } \\
\text { Timber: construction materials production }\end{array}$ & 33 \\
\hline $\begin{array}{l}\text { Erica scoparia L. subsp. } \\
\text { scoparia (Ericaceae) } \\
\text { BCN46083 }\end{array}$ & $\begin{array}{l}\text { Bruc, bruc d'escombra, bruc } \\
\text { de fer escombres, bruc de } \\
\text { Ilei, bruc femella, bruga, }\end{array}$ & $A E, M O$ & $\begin{array}{l}\text { Aerial part. } \\
\text { Stem }\end{array}$ & $\begin{array}{l}\text { Artisanal: broom elaboration } \\
\text { Artisanal: smoking pipe elaboration } \\
\text { Ludic } \\
\text { Ornamental: gardening }\end{array}$ & 44 \\
\hline $\begin{array}{l}\text { Erigeron acer L. (Asteraceae) } \\
\text { BCN35105 }\end{array}$ & - & MO & Inflorescence & $\begin{array}{l}\text { Agrosilvopastoral management: agricultural/ } \\
\text { horticultural }\end{array}$ & 1 \\
\hline $\begin{array}{l}\text { Eriobotrya japonica (Thunb.) } \\
\text { Lindl. (Rosaceae) BCN29695 }\end{array}$ & Nisprero & $\mathrm{Bl}$ & Leaf & $\begin{array}{l}\text { Ornamental: bouquet elaboration } \\
\text { Other informations: ecological information }\end{array}$ & 2 \\
\hline $\begin{array}{l}\text { Erodium moschatum (L.) } \\
\text { L'Hér. (Geraniaceae) } \\
\text { BCN95590 }\end{array}$ & Rellotges & $\mathrm{Bl}$ & Fruit & Ludic & 3 \\
\hline $\begin{array}{l}\text { Eryngium bourgatii Gouan } \\
\text { (Apiaceae) BCN24881 }\end{array}$ & Espinacal blau & $\mathrm{Rl}$ & Aerial part & Ornamental: bouquet elaborations & 1 \\
\hline $\begin{array}{l}\text { Eryngium campestre L. } \\
\text { (Apiaceae) BCN31274 }\end{array}$ & $\begin{array}{l}\text { Card, card girgoler, } \\
\text { espinacal, espinacalç, } \\
\text { espinacs, galabart, panical }\end{array}$ & $\mathrm{AE}, \mathrm{BI} \mathrm{MO}$ & $\begin{array}{l}\text { Root. Whole } \\
\text { plant }\end{array}$ & $\begin{array}{l}\text { Agrosilvopastoral management: weeds } \\
\text { Folk oral literature } \\
\text { Magic and religious beliefs and practices } \\
\text { Other informations: ecological information }\end{array}$ & 6 \\
\hline
\end{tabular}


Table 1 Non-food and non-medicinal folk plant uses in the territories of Alt Empordà, Montseny and Ripollès (Catalonia), and Formentera and Mallorca (Balearic Islands) (Continued)

\begin{tabular}{|c|c|c|c|c|c|}
\hline $\begin{array}{l}\text { Eucalyptus globulus Labill. } \\
\text { (Myrtaceae) BCN29696 }\end{array}$ & $\begin{array}{l}\text { Calipto, calistro, calistrero, } \\
\text { eucaliptus, eucalitus }\end{array}$ & $\mathrm{AE}, \mathrm{BI}, \mathrm{MO}$ & $\begin{array}{l}\text { Aerial part. } \\
\text { Flower. Leaf. } \\
\text { Whole plant }\end{array}$ & $\begin{array}{l}\text { Agrosilvopastoral management: forestry } \\
\text { Agrosilvopastoral management: honey } \\
\text { obtaining } \\
\text { Domestic: air freshener } \\
\text { Ornamental: bouquet elaboration }\end{array}$ & 7 \\
\hline $\begin{array}{l}\text { Eupatorium cannabinum L. } \\
\text { subsp. cannabinum } \\
\text { (Asteraceae) BCN35520 }\end{array}$ & Altes i mires & $\mathrm{MO}$ & Whole plant & Ornamental: bouquet elaboration & 1 \\
\hline $\begin{array}{l}\text { Euphorbia characias L. } \\
\text { subsp. characias } \\
\text { (Euphorbiaceae) BCN29865 }\end{array}$ & Lleteresa & $\mathrm{AE}$ & $\begin{array}{l}\text { Stem. Whole } \\
\text { plant }\end{array}$ & Repellent & 2 \\
\hline $\begin{array}{l}\text { Euphorbia lathyris L. } \\
\text { (Euphorbiaceae) BCN29866 }\end{array}$ & $\begin{array}{l}\text { Cagamuix, herba talpera, } \\
\text { herba taupera, herba } \\
\text { taupinera }\end{array}$ & $A E, R l$ & $\begin{array}{l}\text { Latex. Leaf. } \\
\text { Whole plant }\end{array}$ & $\begin{array}{l}\text { Magic and religious beliefs and practices } \\
\text { Repellent }\end{array}$ & 6 \\
\hline $\begin{array}{l}\text { Euphorbia sp. } \\
\text { (Euphorbiaceae) }\end{array}$ & Eufòrbia, lletrada, Iletrera & $\mathrm{Bl}$ & $\begin{array}{l}\text { Latex. Not } \\
\text { reported }\end{array}$ & $\begin{array}{l}\text { Agrosilvopastoral management: for hunting } \\
\text { Artisanal: footwear elaboration } \\
\text { Fuel obtaining: plant oil }\end{array}$ & 3 \\
\hline $\begin{array}{l}\text { Fagus sylvatica L. (Fagaceae) } \\
\text { BCN46845 }\end{array}$ & Faig & $\begin{array}{l}\mathrm{AE}, \mathrm{BI} \\
\mathrm{MO}, \mathrm{RI}\end{array}$ & $\begin{array}{l}\text { Stem. Whole } \\
\text { plant }\end{array}$ & $\begin{array}{l}\text { Agrosilvopastoral management: tools for } \\
\text { agricultural practices } \\
\text { Artisanal: musical instrument elaboration } \\
\text { Fuel obtaining: charcoal } \\
\text { Timber: boat manufacturing } \\
\text { Timber: furniture manufacturing }\end{array}$ & 15 \\
\hline $\begin{array}{l}\text { Feijoa sellowiana O.Berg } \\
\text { (Myrtaceae) BCN92988 }\end{array}$ & Feijoa & $\mathrm{Bl}$ & Whole plant & Ornamental: gardening & 1 \\
\hline $\begin{array}{l}\text { Ferula communis L. } \\
\text { (Apiaceae) BCN26849 }\end{array}$ & Canya fèl · lera, canyaferla & $\mathrm{Bl}$ & Leaf. Stem & $\begin{array}{l}\text { Ludic } \\
\text { Other informations: ecological information }\end{array}$ & 3 \\
\hline $\begin{array}{l}\text { Ficus carica L. (Moraceae) } \\
\text { BCN24887 }\end{array}$ & $\begin{array}{l}\text { Figa (infructescence), figa } \\
\text { flor (infructescence), figó } \\
\text { marinenc (infructescence) } \\
\text { figuera, lletrada de figuera } \\
\text { (latex), pàmpol de figuera }\end{array}$ & $\mathrm{AE}, \mathrm{Bl}$ & $\begin{array}{l}\text { Bark. } \\
\text { Infructescence. } \\
\text { Stem. Whole } \\
\text { plant }\end{array}$ & $\begin{array}{l}\text { Agrosilvopastoral management: agricultural/ } \\
\text { horticultural } \\
\text { Folk oral literature } \\
\text { Ludic } \\
\text { Magic and religious beliefs and practices } \\
\text { Other informations: ecological information } \\
\text { Other informations: excipient or adjuvant in } \\
\text { medicinal or food preparationsSmoking } \\
\text { Timber } \\
\text { Timber: wheeled vehicles manufacturing }\end{array}$ & 34 \\
\hline $\begin{array}{l}\text { Foeniculum vulgare Mill. } \\
\text { (Apiaceae) BCN95541 }\end{array}$ & Fonoll & $\mathrm{Bl}$ & Aerial part & $\begin{array}{l}\text { Agrosilvopastoral management: for pig } \\
\text { slaughter }\end{array}$ & 1 \\
\hline $\begin{array}{l}\text { Foeniculum vulgare Mill. } \\
\text { subsp. piperitum (C.Presl) } \\
\text { Ball (Apiaceae) BCN29867 }\end{array}$ & Fonoll & $A E$ & Leaf. Stem & $\begin{array}{l}\text { Agrosilvopastoral management: for pig } \\
\text { slaughter } \\
\text { Domestic: for help at home }\end{array}$ & 2 \\
\hline $\begin{array}{l}\text { Fraxinus angustifolia Vahl } \\
\text { (Oleaceae) BCN46844 }\end{array}$ & $\begin{array}{l}\text { Fleix, fleix femella, fleix } \\
\text { mascle, freixa, freixe }\end{array}$ & $\mathrm{AE}, \mathrm{BI}, \mathrm{MO}$ & $\begin{array}{l}\text { Stem. Whole } \\
\text { plant }\end{array}$ & $\begin{array}{l}\text { Agrosilvopastoral management: tools for } \\
\text { agricultural practices } \\
\text { Artisanal: broom elaboration } \\
\text { Artisanal: cane elaboration } \\
\text { Ludic } \\
\text { Other informations: ecological information } \\
\text { Timber: boat manufacturing } \\
\text { Timber: wheeled vehicles manufacturing } \\
\text { Timber: furniture manufacturing }\end{array}$ & 21 \\
\hline $\begin{array}{l}\text { Fraxinus excelsior L. } \\
\text { (Oleaceae) BCN29698 }\end{array}$ & Freixe & $\mathrm{AE}, \mathrm{MO}, \mathrm{RI}$ & $\begin{array}{l}\text { Leaf. Stem. } \\
\text { Whole plant }\end{array}$ & $\begin{array}{l}\text { Artisanal: cane elaboration } \\
\text { Artisanal: musical instrument elaboration } \\
\text { Domestic: for help at home } \\
\text { Magic and religious beliefs and practices } \\
\text { Ornamental: gardening } \\
\text { Timber: boat manufacturing } \\
\text { Timber: wheeled vehicles manufacturing }\end{array}$ & 11 \\
\hline Fuchsia sp. (Onagraceae) & $\begin{array}{l}\text { Pendientes de la reina } \\
\text { (Spanish) }\end{array}$ & $\mathrm{Bl}$ & Whole plant & Ornamental: gardening & 1 \\
\hline
\end{tabular}


Table 1 Non-food and non-medicinal folk plant uses in the territories of Alt Empordà, Montseny and Ripollès (Catalonia), and Formentera and Mallorca (Balearic Islands) (Continued)

\begin{tabular}{|c|c|c|c|c|c|}
\hline $\begin{array}{l}\text { Fumaria officinalis L. } \\
\text { (Papaveraceae) BCN72725 }\end{array}$ & Fumaterra & $\mathrm{Bl}$ & Whole plant & Other informations: harvesting and/or selling & 1 \\
\hline Gaillardia sp. (Asteraceae) & Gallàrdia & $\mathrm{Bl}$ & Whole plant & Ornamental: gardening & 1 \\
\hline $\begin{array}{l}\text { Galanthus nivalis L. } \\
\text { (Amaryllidaceae) BCN27249 }\end{array}$ & $\begin{array}{l}\text { Flor de neu, herba de cap } \\
\text { blanc, herba de neu, } \\
\text { llàgrimes de Sant Josep }\end{array}$ & $\mathrm{MO}, \mathrm{Rl}$ & Aerial part & Ornamental: bouquet elaboration & 5 \\
\hline $\begin{array}{l}\text { Galium aparine L. } \\
\text { (Rubiaceae) BCN97749 }\end{array}$ & Rèvola & $\mathrm{Bl}$ & Fruit & Other informations: ecological information & 1 \\
\hline $\begin{array}{l}\text { Galium verum L. (Rubiaceae) } \\
\text { BCN39982 }\end{array}$ & $\begin{array}{l}\text { Herba de Sant Joan, } \\
\text { santjoan }\end{array}$ & $A E, R l$ & $\begin{array}{l}\text { Aerial part. Not } \\
\text { reported }\end{array}$ & $\begin{array}{l}\text { Ornamental: bouquet elaboration } \\
\text { Other informations: ecological information }\end{array}$ & 2 \\
\hline $\begin{array}{l}\text { Genista tricuspidata Desf. } \\
\text { subsp. sparsiflora (Ball) } \\
\text { Maire (Fabaceae) BCN } \\
95565\end{array}$ & Gatova & $\mathrm{Bl}$ & $\begin{array}{l}\text { Aerial part. } \\
\text { Whole plant }\end{array}$ & $\begin{array}{l}\text { Agrosilvopastoral management: for pig } \\
\text { slaughter } \\
\text { Folk oral literature }\end{array}$ & 5 \\
\hline $\begin{array}{l}\text { Gentiana lutea L. } \\
\text { (Gentianaceae) BCN24893 }\end{array}$ & Llenciana & $\mathrm{Rl}$ & $\begin{array}{l}\text { Aerial part. } \\
\text { Root }\end{array}$ & $\begin{array}{l}\text { Ornamental: bouquet elaboration } \\
\text { Other informations: harvesting and/or selling }\end{array}$ & 2 \\
\hline $\begin{array}{l}\text { Ginkgo biloba L. } \\
\text { (Ginkgoaceae) BCN62881 }\end{array}$ & Ginkgo, ginkgo biloba & $\mathrm{Bl}, \mathrm{MO}$ & Whole plant & $\begin{array}{l}\text { Ornamental: gardening } \\
\text { Other informations: ecological information }\end{array}$ & 2 \\
\hline $\begin{array}{l}\text { Gladiolus italicus Mill. } \\
\text { (Iridiaceae) BCN56421 }\end{array}$ & $\begin{array}{l}\text { Espadelles, espasa, espaseta, } \\
\text { gladiol, xupes }\end{array}$ & $\mathrm{Bl}, \mathrm{Rl}$ & $\begin{array}{l}\text { Aerial part. } \\
\text { Flower. Whole } \\
\text { plant }\end{array}$ & $\begin{array}{l}\text { Ornamental: bouquet elaboration } \\
\text { Ornamental: gardening }\end{array}$ & 5 \\
\hline Gladiolus sp. (Iridiaceae) & Gladiol & $\mathrm{Bl}$ & Aerial part & Ornamental: bouquet elaboration & 1 \\
\hline $\begin{array}{l}\text { Gleditsia triachanthos L. } \\
\text { (Fabaceae) BCN50767 }\end{array}$ & Escàcia & $\mathrm{AE}$ & Stem & Domestic: for help at home & 1 \\
\hline $\begin{array}{l}\text { Globularia alypum L. } \\
\text { (Globulariaceae) BCN29868 }\end{array}$ & Cossiada, herba cossiada & $\mathrm{Bl}$ & $\begin{array}{l}\text { Leaf. Whole } \\
\text { plant }\end{array}$ & $\begin{array}{l}\text { Folk oral literature } \\
\text { Other informations: ecological information }\end{array}$ & 2 \\
\hline $\begin{array}{l}\text { Glycyrrhiza glabra L. } \\
\text { (Fabaceae) BCN47276 }\end{array}$ & Regalíssia & $\mathrm{AE}$ & Root & Smoking & 1 \\
\hline $\begin{array}{l}\text { Gomphocarpus fruticosus (L.) } \\
\text { W.T.Aiton (Asclepiadaceae) } \\
\text { BCN29987 }\end{array}$ & Tintorell & $\mathrm{AE}$ & Root & Agrosilvopastoral management: for fishing & 1 \\
\hline $\begin{array}{l}\text { Gossypium sp. (Malvaceae) } \\
\text { BCN51688 }\end{array}$ & Cotó & $\mathrm{AE}, \mathrm{BI}$ & Fruit. Seed hair & $\begin{array}{l}\text { Agrosilvopastoral management: for fishing } \\
\text { Magic and religious beliefs and practices } \\
\text { Other informations: ecological information }\end{array}$ & 4 \\
\hline $\begin{array}{l}\text { Hedera helix L. (Araliaceae) } \\
\text { BCN29869 }\end{array}$ & Heura & $\begin{array}{l}\mathrm{AE}, \mathrm{Bl} \\
\mathrm{MO}, \mathrm{RI}\end{array}$ & $\begin{array}{l}\text { Aerial part. } \\
\text { Flower. Leaf. } \\
\text { Whole plant }\end{array}$ & $\begin{array}{l}\text { Agrosilvopastoral management: honey } \\
\text { obtaining } \\
\text { Cosmetic } \\
\text { Domestic: for help at home } \\
\text { Magic and religious beliefs and practices } \\
\text { Ornamental: gardening } \\
\text { Other informations: use not remembered }\end{array}$ & 22 \\
\hline $\begin{array}{l}\text { Helianthus tuberosus L. } \\
\text { (Asteraceae) BCN46069 }\end{array}$ & $\begin{array}{l}\text { Gira-sol de patata, miqueler, } \\
\text { nyama, nyàmera, ramon, } \\
\text { setembre, trumfa nyama }\end{array}$ & $\begin{array}{l}\mathrm{AE}, \mathrm{BI} M O, \\
\mathrm{Rl}\end{array}$ & $\begin{array}{l}\text { Aerial part. } \\
\text { Whole plant }\end{array}$ & $\begin{array}{l}\text { Agrosilvopastoral management: tools for } \\
\text { agricultural practices } \\
\text { Ornamental: bouquet elaboration }\end{array}$ & 6 \\
\hline $\begin{array}{l}\text { Helichrysum stoechas (L.) } \\
\text { Moench (Asteraceae) } \\
\text { BCN29872 }\end{array}$ & $\begin{array}{l}\text { Éternelle (French), flor de } \\
\text { Corpus, flor de Sant Joan, } \\
\text { perpètua, sempreviva }\end{array}$ & $\mathrm{AE}, \mathrm{BI}, \mathrm{MO}$ & $\begin{array}{l}\text { Aerial part. } \\
\text { Inflorescence }\end{array}$ & $\begin{array}{l}\text { Magic and religious beliefs and practices } \\
\text { Ornamental: bouquet elaboration } \\
\text { Repellent }\end{array}$ & 38 \\
\hline $\begin{array}{l}\text { Helleborus foetidus L. } \\
\text { (Ranunculaceae) BCN32163 }\end{array}$ & Margívol, marxívol & $\mathrm{MO}$ & Fruit. Leaf & $\begin{array}{l}\text { Folk oral literature } \\
\text { Ornamental: bouquet elaboration }\end{array}$ & 9 \\
\hline $\begin{array}{l}\text { Helleborus viridis L. } \\
\text { (Ranunculaceae) BCN24899 }\end{array}$ & Mengívol, marxívol & $\mathrm{Rl}$ & $\begin{array}{l}\text { Aerial part. } \\
\text { Fruit }\end{array}$ & Magic and religious beliefs and practices & 3 \\
\hline $\begin{array}{l}\text { Herniaria glabra L. } \\
\text { (Caryophyllaceae) } \\
\text { BCN32161 }\end{array}$ & $\begin{array}{l}\text { Herba de cent en grana, } \\
\text { santaengrana }\end{array}$ & $\mathrm{MO}$ & Whole plant & Folk oral literature & 2 \\
\hline
\end{tabular}


Table 1 Non-food and non-medicinal folk plant uses in the territories of Alt Empordà, Montseny and Ripollès (Catalonia), and Formentera and Mallorca (Balearic Islands) (Continued)

\begin{tabular}{|c|c|c|c|c|c|}
\hline $\begin{array}{l}\text { Herniaria hirsuta L. subsp. } \\
\text { cinerea (DC.) Cout. } \\
\text { (Caryophyllaceae) } \\
\text { BCN45660 }\end{array}$ & $\begin{array}{l}\text { Herba de trencapreda, } \\
\text { trencapedra }\end{array}$ & $\mathrm{BI}$ & $\begin{array}{l}\text { Aerial part. } \\
\text { Whole plant }\end{array}$ & $\begin{array}{l}\text { Magic and religious beliefs and practices } \\
\text { Other informations: ecological information }\end{array}$ & 5 \\
\hline $\begin{array}{l}\text { Hibiscus syriacus L. } \\
\text { (Malvaceae) BCN47686 }\end{array}$ & Altea & $\mathrm{Bl}$ & $\begin{array}{l}\text { Flower. Root. } \\
\text { Whole plant }\end{array}$ & $\begin{array}{l}\text { Ornamental: gardening } \\
\text { Other informations: use not remembered }\end{array}$ & 3 \\
\hline $\begin{array}{l}\text { Hippocrepis balearica Jacq. } \\
\text { (Fabaceae) BCN21809 }\end{array}$ & $\begin{array}{l}\text { Viola de penyal, violeta de } \\
\text { penyal }\end{array}$ & $\mathrm{Bl}$ & Whole plant & $\begin{array}{l}\text { Agrosilvopastoral management: tools for } \\
\text { agricultural practices } \\
\text { Folk oral literature } \\
\text { Ornamental: gardening }\end{array}$ & 3 \\
\hline $\begin{array}{l}\text { Hirschfeldia incana (L.) Lagr.- } \\
\text { Foss. (Brassicaceae) } \\
\text { BCN39984 }\end{array}$ & Ravenissa & $\mathrm{AE}$ & Whole plant & Other informations: use not remembered & 1 \\
\hline $\begin{array}{l}\text { Hordeum murinum L. subsp. } \\
\text { leporinum (Link) Arcang. } \\
\text { (Poaceae) BCN79674 }\end{array}$ & $\begin{array}{l}\text { Cebada (Spanish), fletxa, } \\
\text { malta, maragall, ordi }\end{array}$ & $\mathrm{Bl}, \mathrm{MO}$ & $\begin{array}{l}\text { Inflorescence. } \\
\text { Whole plant }\end{array}$ & $\begin{array}{l}\text { Folk oral literature } \\
\text { Ludic }\end{array}$ & 8 \\
\hline $\begin{array}{l}\text { Hordeum vulgare L. } \\
\text { (Poaceae) BCN46843 }\end{array}$ & Ordi & $A E, B I, R I$ & $\begin{array}{l}\text { Aerial part. } \\
\text { Fruit. Stem. } \\
\text { Whole plant }\end{array}$ & $\begin{array}{l}\text { Agrosilvopastoral management: agricultural/ } \\
\text { horticultural } \\
\text { Agrosilvopastoral management: honey } \\
\text { obtaining } \\
\text { Artisanal: footwear elaboration } \\
\text { Cosmetic } \\
\text { Magic and religious beliefs and practices } \\
\text { Other informations: harvesting and/or selling }\end{array}$ & 12 \\
\hline $\begin{array}{l}\text { Howea forsteriana (F.Muell.) } \\
\text { Becc. (Arecaceae) } \\
\text { BCN129083 }\end{array}$ & Quènsia & $\mathrm{Bl}$ & Whole plant & Ornamental: gardening & 1 \\
\hline $\begin{array}{l}\text { Hoya carnosa (L.f.) R.Br. } \\
\text { (Asclepiadaceae) } \\
\text { BCN104268 }\end{array}$ & Cerera & $\mathrm{Bl}$ & $\begin{array}{l}\text { Flower. Whole } \\
\text { plant }\end{array}$ & $\begin{array}{l}\text { Domestic: for help at home } \\
\text { Ornamental: gardening }\end{array}$ & 3 \\
\hline $\begin{array}{l}\text { Hydrangea macrophylla } \\
\text { (Thunb.) Ser. } \\
\text { (Hydrangeaceae) BC800370 }\end{array}$ & Hortènsia & $\mathrm{MO}$ & Flower & Magic and religious beliefs and practices & 1 \\
\hline $\begin{array}{l}\text { Hydrangea } \\
\text { sp. (Hydrangeaceae) }\end{array}$ & Hortènsia & $\mathrm{Bl}$ & Whole plant & $\begin{array}{l}\text { Agrosilvopastoral management: tools for } \\
\text { agricultural practices } \\
\text { Ornamental: gardening }\end{array}$ & 3 \\
\hline $\begin{array}{l}\text { Hyoscyamus albus L. } \\
\text { (Solanaceae) BCN } 31277\end{array}$ & Herba de capceta & $\mathrm{Bl}$ & $\begin{array}{l}\text { Leaf. Whole } \\
\text { plant }\end{array}$ & $\begin{array}{l}\text { Other informations: harvesting and/or selling } \\
\text { Smoking }\end{array}$ & 2 \\
\hline $\begin{array}{l}\text { Hypericum balearicum L. } \\
\text { (Clusiaceae) BCN95574 }\end{array}$ & Espeta joana & $\mathrm{Bl}$ & $\begin{array}{l}\text { Aerial part. } \\
\text { Whole plant }\end{array}$ & $\begin{array}{l}\text { Other informations: ecological information } \\
\text { Repellent }\end{array}$ & 3 \\
\hline $\begin{array}{l}\text { Hypericum perforatum L. } \\
\text { (Clusiaceae) BCN29874 }\end{array}$ & $\begin{array}{l}\text { Herba de Sant Joan, herba } \\
\text { dels set caps, hipèric, } \\
\text { hipèricum, trescalam }\end{array}$ & $\mathrm{AE}, \mathrm{MO}, \mathrm{RI}$ & $\begin{array}{l}\text { Aerial part. Not } \\
\text { reported. } \\
\text { Whole plant }\end{array}$ & $\begin{array}{l}\text { Cosmetic } \\
\text { Magic and religious beliefs and practices } \\
\text { Ornamental: bouquet elaboration } \\
\text { Other informations: harvesting and/or selling } \\
\text { Other informations: purchased in commerce }\end{array}$ & 8 \\
\hline $\begin{array}{l}\text { Ilex aquifolium L. } \\
\text { (Aquifoliaceae) BCN29876 }\end{array}$ & $\begin{array}{l}\text { Boix grèvol, flor de Nadal, } \\
\text { grèvol }\end{array}$ & $\mathrm{AE}, \mathrm{MO}, \mathrm{RI}$ & $\begin{array}{l}\text { Aerial part. } \\
\text { Bark. Resin. } \\
\text { Stem. Whole } \\
\text { plant }\end{array}$ & $\begin{array}{l}\text { Agrosilvopastoral management: tools for } \\
\text { agricultural practices } \\
\text { Agrosilvopastoral management: for hunting } \\
\text { Artisanal: cane elaboration } \\
\text { Magic and religious beliefs and practices } \\
\text { Ornamental: bouquet elaboration } \\
\text { Ornamental: gardening }\end{array}$ & 25 \\
\hline $\begin{array}{l}\text { Inula viscosa (L.) Aiton } \\
\text { (Asteraceae) BCN31279 }\end{array}$ & $\begin{array}{l}\text { Herba de puces, } \\
\text { matavinyes, olivarda }\end{array}$ & $A E, B I$ & $\begin{array}{l}\text { Aerial part. } \\
\text { Inflorescence. } \\
\text { Leaf. Not } \\
\text { reported. Stem. } \\
\text { Whole plant }\end{array}$ & $\begin{array}{l}\text { Agrosilvopastoral management: for hunting } \\
\text { Artisanal: broom elaboration } \\
\text { Folk oral literature } \\
\text { Magic and religious beliefs and practices } \\
\text { Other informations: ecological information } \\
\text { Other informations: use not remembered } \\
\text { Repellent }\end{array}$ & 15 \\
\hline
\end{tabular}


Table 1 Non-food and non-medicinal folk plant uses in the territories of Alt Empordà, Montseny and Ripollès (Catalonia), and Formentera and Mallorca (Balearic Islands) (Continued)

Ipomoea batatas (L.) Lam. Monais, moneiato, moniato, B (Convolvulaceae) BCN50213 moniajo
Juncus effussus L.

(Juncaceae) BCN39991

Juniperus communis L. (Cupressaceae) BCN24910

Juniperus communis L.

subsp. communis

(Cupressaceae) BCN29878

Juniperus oxycedrus L.

(Cupressaceae) BCN29879

Juniperus oxycedrus L.

subsp. oxycedrus

(Cupressaceae) BCN29879

Juniperus phoenicea L. (Cupressaceae) BCN36078

Koelreuteria paniculata

Laxm. (Sapindaceae)

BCN63272 u, lliri de marge,

Lliri de rec
Xicaranda
Jasmí
Bejamí, gessamí, geramí,
llessamí
Anoguer, anou (fruit),
noguer, noguera, nou
(fruit), nou verda (fruit),
nogalina (elaborated
product)

Jonc, jonc marí

Ginebre, ginebró, ginebró de jardí, oli de ginebre (elaborated product)

Ginebre, ginebró

$\mathrm{AE}$

Savina, savinó (cone), sivina B

Saboner
Tuber. Whole plant

Aerial part. Flower. Whole plant

$\mathrm{AE}, \mathrm{BI}$ $\mathrm{MO}, \mathrm{RI}$ Leaf. Stem. Whole plant

Aerial part Stem

\section{Fructification.} tem. Whole plant
Agrosilvopastoral management: agricultural/

horticultural

Folk oral literature

Ornamental: bouquet elaboration

Other informations: ecological information

Magic and religious beliefs and practices

Ornamental: bouquet elaboration

Ornamental: gardening

Ornamental: bouquet elaboration

Ornamental: gardening

Ornamental: gardening

Other informations: ecological information

26

agricultural practices

Artisanal: cookware elaboration

Cosmetic

Domestic: for help at home

Folk oral literature

Magic and religious beliefs and practices

Other informations: ecological information

Textile: dyer

Timber

Timber: boat manufacturing

Timber: furniture manufacturing
Aerial part. Fruictification.

Stem

Whole plant

Fruictification. Not reported.

Root. Stem. Whole plant

Agrosilvopastoral management: for fishing

Artisanal: basketry

Domestic: for help at home

Agrosilvopastoral management: agricultural/

horticultural

Domestic: for help at home

Folk oral literature

Ornamental: gardening

Other informations

Agrosilvopastoral management: tools for agricultural practices

Magic and religious beliefs and practices

Other informations: ecological information

Other informations: ecological information

Artisanal: musical instrument elaboration

isanal: smoking pipe elaboration

Domestic: for help at home

Fuel obtaining: firewood

Ornamental: gardening

Other informations: ecological information

Fruictification. Agrosilvopastoral management: tools for

agricultural practices

Artisanal: cane elaboration

Ludic

Timber

Domestic: for help at home

4

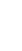

(1)


Table 1 Non-food and non-medicinal folk plant uses in the territories of Alt Empordà, Montseny and Ripollès (Catalonia), and Formentera and Mallorca (Balearic Islands) (Continued)

\begin{tabular}{|c|c|c|c|c|c|}
\hline $\begin{array}{l}\text { Lagenaria siceraria (Molina) } \\
\text { Standl. (Cucurbitaceae) } \\
\text { BCN50212 }\end{array}$ & Carabassa, catija & $\mathrm{AE}, \mathrm{BI}$ & Fruit & $\begin{array}{l}\text { Agrosilvopastoral management: tools for } \\
\text { agricultural practices } \\
\text { Artisanal: cookware elaboration } \\
\text { Domestic: for help at home } \\
\text { Ornamental: bouquet elaboration }\end{array}$ & 5 \\
\hline $\begin{array}{l}\text { Lagurus ovatus L. (Poaceae) } \\
\text { BCN31281 }\end{array}$ & - & $\mathrm{AE}$ & Aerial part & Ornamental: bouquet elaboration & 2 \\
\hline $\begin{array}{l}\text { Lantana camara L. } \\
\text { (Verbenaceae) BCN } 40081\end{array}$ & Bandera espanyola & $\mathrm{Bl}$ & Whole plant & Ornamental: gardening & 2 \\
\hline $\begin{array}{l}\text { Lathyrus sativus L. } \\
\text { (Fabaceae) BCN } 50775\end{array}$ & Guixa (fruit) & $\mathrm{Bl}$ & Whole plant & $\begin{array}{l}\text { Agrosilvopastoral management: agricultural/ } \\
\text { horticultural }\end{array}$ & 1 \\
\hline $\begin{array}{l}\text { Launaea cervicornis (Boiss.) } \\
\text { Font Quer \& Rothm. } \\
\text { (Asteraceae) BCN88711 }\end{array}$ & Gatovell & $\mathrm{Bl}$ & Aerial part & $\begin{array}{l}\text { Agrosilvopastoral management: tools for } \\
\text { agricultural practices }\end{array}$ & 1 \\
\hline $\begin{array}{l}\text { Laurus nobilis L. (Lauraceae) } \\
\text { BCN29880 }\end{array}$ & Llaurer, Ilor, llorer, llort & $\begin{array}{l}\mathrm{AE}, \mathrm{Bl} \\
\mathrm{MO}, \mathrm{RI}\end{array}$ & $\begin{array}{l}\text { Aerial part. } \\
\text { Leaf. Seed. } \\
\text { Stem. Whole } \\
\text { plant }\end{array}$ & $\begin{array}{l}\text { Agrosilvopastoral management: tools for } \\
\text { agricultural practices } \\
\text { Artisanal: cane elaboration } \\
\text { Cosmetic } \\
\text { Domestic: air freshener } \\
\text { Domestic: for help at home } \\
\text { Folk oral literature } \\
\text { Magic and religious beliefs and practices } \\
\text { Other informations: ecological information } \\
\text { Repellent } \\
\text { Timber: construction materials production }\end{array}$ & 49 \\
\hline $\begin{array}{l}\text { Lavandula angustifolia Mill. } \\
\text { (Lamiaceae) BCN29881 }\end{array}$ & Espígol, lavanda & $A E, B I$ & $\begin{array}{l}\text { Aerial part. } \\
\text { Flower. Whole } \\
\text { plant }\end{array}$ & $\begin{array}{l}\text { Domestic: air freshener } \\
\text { Ornamental: bouquet elaboration } \\
\text { Ornamental: gardening } \\
\text { Other informations: ecological information }\end{array}$ & 4 \\
\hline $\begin{array}{l}\text { Lavandula angustifolia Mill. } \\
\text { subsp. angustifolia } \\
\text { (Lamiaceae) BCN29715 }\end{array}$ & $\begin{array}{l}\text { Barballó, bermelló, espígol, } \\
\text { lavanda }\end{array}$ & $\mathrm{Rl}$ & Aerial part & Domestic: air freshener & 2 \\
\hline $\begin{array}{l}\text { Lavandula dentata L. } \\
\text { (Lamiaceae) BCN24913 }\end{array}$ & $\begin{array}{l}\text { Candelero, colònia, lavanda, } \\
\text { gal.landa }\end{array}$ & $\mathrm{AE}, \mathrm{Bl}, \mathrm{MO}$ & $\begin{array}{l}\text { Aerial part. } \\
\text { Flower. Whole } \\
\text { plant }\end{array}$ & $\begin{array}{l}\text { Agrosilvopastoral management: tools for } \\
\text { agricultural practices } \\
\text { Cosmetic } \\
\text { Domestic: air freshener }\end{array}$ & 9 \\
\hline $\begin{array}{l}\text { Lavandula latifolia Medik. } \\
\text { (Lamiaceae) BCN29882 }\end{array}$ & $\begin{array}{l}\text { Barballó, bermelló, espígol, } \\
\text { lavanda }\end{array}$ & $\mathrm{AE}, \mathrm{Bl}, \mathrm{MO}$ & $\begin{array}{l}\text { Aerial part. } \\
\text { Flower. Leaf. } \\
\text { Whole plant }\end{array}$ & $\begin{array}{l}\text { Agrosilvopastoral management: honey } \\
\text { obtaining } \\
\text { Agrosilvopastoral management: tools for } \\
\text { agricultural practices } \\
\text { Cosmetic } \\
\text { Domestic: air freshener } \\
\text { Ornamental: bouquet elaboration } \\
\text { Ornamental: gardening } \\
\text { Repellent } \\
\text { Smoking }\end{array}$ & 30 \\
\hline $\begin{array}{l}\text { Lavandula stoechas L. } \\
\text { (Lamiaceae) BCN126622 }\end{array}$ & Cap d'ase, lavanda, tomanyí & $\mathrm{Bl}, \mathrm{MO}$ & $\begin{array}{l}\text { Aerial part. } \\
\text { Flower }\end{array}$ & $\begin{array}{l}\text { Agrosilvopastoral management: tools for } \\
\text { agricultural practices } \\
\text { Cosmetic } \\
\text { Smoking }\end{array}$ & 7 \\
\hline $\begin{array}{l}\text { Lavandula stoechas L. } \\
\text { subsp. stoechas (Lamiaceae) } \\
\text { BCN29883 }\end{array}$ & Timó & $A E$ & $\begin{array}{l}\text { Aerial part. } \\
\text { Flower }\end{array}$ & $\begin{array}{l}\text { Domestic: air freshener } \\
\text { Ornamental: bouquet elaboration } \\
\text { Smoking }\end{array}$ & 4 \\
\hline $\begin{array}{l}\text { Lavatera arborea L. } \\
\text { (Malvaceae) BCN95546 }\end{array}$ & Malva & $\mathrm{AE}, \mathrm{Bl}$ & $\begin{array}{l}\text { Aerial part. } \\
\text { Flower }\end{array}$ & $\begin{array}{l}\text { Agrosilvopastoral management: tools for } \\
\text { agricultural practices } \\
\text { Magic and religious beliefs and practices }\end{array}$ & 2 \\
\hline $\begin{array}{l}\text { Lavatera cretica L. } \\
\text { (Malvaceae) BCN44944 }\end{array}$ & Vauma & $\mathrm{Bl}$ & Whole plant & $\begin{array}{l}\text { Agrosilvopastoral management: weeds } \\
\text { Folk oral literature } \\
\text { Other informations: ecological information }\end{array}$ & 4 \\
\hline
\end{tabular}


Table 1 Non-food and non-medicinal folk plant uses in the territories of Alt Empordà, Montseny and Ripollès (Catalonia), and Formentera and Mallorca (Balearic Islands) (Continued)

Lens culinaris Medik.
(Fabaceae) BCN 29990
Leontodon taraxacoides
Hoppe \& Hornsch.
(Asteraceae) BCN48777
Leontopodium alpinum
Guss. subsp. alpinum
(Asteraceae) BCN34286
Leucanthemum vulgare
(Vaill.) Lam (Asteraceae)
BCN29721
Leuzea conifera (L.) DC. in
Lam. \& DC. (Asteraceae)
BCN29884
Ligustrum vulgare L.
(Oleaceae) BCN24915
Lilium candidum L.
(Liliaceae) BCN46841

Lilium martagon L. (Liliaceae) BCN24917

Lilium pyrenaicum Gouan (Liliaceae) BCN24918

Limonium virgatum (Willd.) Fourr. (Plumbaginaceae) BCN39989

Limonium vulgare Mill. subsp. serotinum (Rchb.) Gams (Plumbaginaceae) BCN31282

Linaria cymbalaria (L.) Mill (Plantaginaceae) BCN29885

Linum usitatissimum L. (Linaceae) BCN47281

Linum usitatissimum L. subsp. angustifolia (Huds.)

Thell. (Linaceae) BCN107485

Linum usitatissimum L.

subsp. usitatissimum

(Linaceae) BCN32155

Lippia triphylla (L'Hér.)

O.Kuntze (Verbenaceae)

BCN29886

Lolium rigidum Gaudin

(Poaceae) BCN31283

Lonicera etrusca Santi

(Caprifoliaceae) BCN31285

Llentia Bl

Angelets, pixallits

|

$\mathrm{MO}$

Whole plant

Aerial part

Agrosilvopastoral management: agricultural/ horticultural

Herba de neu

AE Aerial part

Ornamental: bouquet elaboration

1

Margarida, margarida

borda, margarida de camp,

AE, RI Aerial part

Ludic

Ornamental: bouquet elaboration

margarida de bosc

Cigales, nyigo-nyigo, pinya de Sant Joan, polles

$A E, M O \quad$ Aerial part

Ornamental: bouquet elaboration

4

Escanyacabres, olivereta

RI Aerial part

Azucena (Spanish), Iliri, Iliri blanc, Iliri blanc de Sant Joan, Iliri de Sant Antoni, lliri de Sant Joan

Consolta, macròlic, marcòlic vermell

$\mathrm{AE}, \mathrm{BI}, \quad$ Aerial part.

$\mathrm{MO}, \mathrm{RI} \quad$ Flower. Whole

plant

Aerial part.

Flower.

Seedling

Consolta, consolta groga, Rl

Aerial part.

Whole plant

marcòlic groc

Patarres

Patarres

AE

Aerial part

mental: bouquet elaboration

Ornamental: gardening

Ornamental: bouquet elaboration

Picardia, setembrina

Lli, Ilinc, Ilim, Ilinosa, oli de llinosa (elaborated product)

AE $\quad$ Aerial part

Magic and religious beliefs and practices

1

AE, BI, MO Whole plant

Magic and religious beliefs and practices Ornamental: gardening

$\mathrm{AE}, \mathrm{BI} \quad$ Aerial part. Not Domestic: paint elaboration reported. Seed. Folk oral literature

seed oil. Whole Textile: fibre or cloth elaboration plant

Herba feridora

$\mathrm{MO}$

Seed

Ornamental: bouquet elaboration

Bri (fibre), Ili, llinosa

$\mathrm{MO}$

Aerial part

Cosmetic

1

Herba lluïsa, marialluïsa

$\mathrm{AE}, \mathrm{BI} \quad$ Aerial part. Leaf. Whole plant

Agrosilvopastoral management: agricultural/ horticultural

Domestic: air freshener

Other informations: ecological information Other informations: excipient or adjuvant in medicinal or food preparations

Margai, margall

$\mathrm{BI}$

Not reported

Folk oral literature

Xuclamel

AE

Stem

Artisanal: smoking pipe elaboration 
Table 1 Non-food and non-medicinal folk plant uses in the territories of Alt Empordà, Montseny and Ripollès (Catalonia), and Formentera and Mallorca (Balearic Islands) (Continued)

\begin{tabular}{ll}
\hline Lonicera implexa Aiton & Mistreres, lligabosc, \\
(Caprifoliaceae) BCN31286 & madreselva (Spanish), \\
& mataselva, rotaboc, \\
& rotaboig, xuclamel
\end{tabular}

Lonicera peryclimenum L.

(Caprifoliaceae) BCN26736

Lophophora williamsii (Lem. ex Salm-Dyck) J.M. Coult. (Cactaceae) BCN129082

Luffa cylindrica (L.) M.Roem. (Cucurbitaceae) BCN-E-78

Lunaria annua L.

(Brassicaceae) BCN56009

Lunaria annua L. subsp. annua (Brassicaceae) BCN31287

Lupinus albus L. (Fabaceae) BCN-E-79

\section{Lycopodium clavatum L.} (Lycopodiaceae) BCN31748

Lygeum spartum Loefl. ex L. (Poaceae) BCN 27831

Magnolia grandiflora L. (Magnoliaceae) BCN64396

Malva sylvestris $\mathrm{L}$. (Malvaceae) BCN29889

Mammillaria sp. (Cactaceae)

Mandragora autumnalis

Bertol. (Solanaceae)

BCN52434

Mantisalca salmantica (L.) Briq. \& Cavill. (Asteraceae) BCN24925

Marrubium vulgare $\mathrm{L}$. (Lamiaceae) BCN72729

Matricaria chamomilla L. (Asteraceae) BCN29890

Matthiola sinuata (L.) R.Br. (Brassicaceae) BCN50218

Musgo (Spanish)

\section{Lligabosc, mareselva}

Peyote (Spanish)

Carabassera d'esponges, fregall (fruit), fregall de carabassera (endocarp), fregaller

Flor de plata

Flor de plata, medallons, moneda del Papa

Llobins, tramusset, tramús

Espart

Magnòlia

Malva, malva de prat, vauma

\section{Mamillària}

Mandràgora

Baleges, cabeçuda, granera

Barrombí, malrobí

Camamilla, camamilla de I'hort, camamilla dolça, mançanilla

Violer

$\begin{aligned} \mathrm{AE}, \mathrm{BI}, \mathrm{MO} & \text { Aerial part. } \\ & \text { Flower. Not } \\ & \text { reported. Stem. } \\ & \text { Whole plant }\end{aligned}$

Agrosilvopastoral management: tools for agricultural practices

Artisanal: smoking pipe elaboration

Folk oral literature

Ludic

Ornamental: gardening

Other informations: ecological information

Other informations: use not remembered

Repellent

Smoking

Magic and religious beliefs and practices

Ornamental: bouquet elaboration

Magic and religious beliefs and practices

Whole plant

Ornamental: gardening

Other informations: harvesting and/or selling

$\mathrm{BI}, \mathrm{MO} \quad$ Endocarp. Fruit. Domestic: for help at home

Mesocarp +

endocarp

RI Aerial part

Ornamental: bouquet elaboration

AE, MO Aerial part.

Whole plant

Ornamental: bouquet elaboration

Seed. Whole

plant

Agrosilvopastoral management: agricultural/ horticultural

Domestic: for help at home

BI $\quad$ Aerial part

Agrosilvopastoral management: tools for agricultural practices

BI Not reported Artisanal: basketry

MO Flower. Leaf. Magic and religious beliefs and practices Whole plant Ornamental: gardening

$\mathrm{AE}, \mathrm{Bl} \quad$ Aerial part.

Folk oral literature

$\mathrm{MO}, \mathrm{RI} \quad$ Flower. Leaf.

Whole plant

Magic and religious beliefs and practices

Other informations: ecological information

BI Not reported. Ornamental: gardening Whole plant

Other informations: use not remembered

BI Whole plant

Folk oral literature

Domestic: for help at home

Aerial part. Seed

Agrosilvopastoral management: honey obtaining

Domestic: for help at home

Magic and religious beliefs and practices Other informations: ecological information Repellent

$A E, M O, R I$ Aerial part. Cosmetic

Ornamental: bouquet elaboration

AE $\quad$ Aerial part

Ornamental: bouquet elaboration Ornamental: gardening 
Table 1 Non-food and non-medicinal folk plant uses in the territories of Alt Empordà, Montseny and Ripollès (Catalonia), and Formentera and Mallorca (Balearic Islands) (Continued)

Medicago sativa L. (Fabaceae) BCN29891

Alfals, aufaus, userda

\author{
(Fabaceae) BCN29891
}

Melia azedarach L. (Meliaceae) BCN97016

Melilotus sulcatus Desf. (Fabaceae) BCN27973

Melissa officinalis L. (Lamiaceae) BCN29731

Melissa officinalis L. subsp.
officinalis (Lamiaceae)
BCN29731

Mentha aquatica L. (Lamiaceae) BCN103554

Mentha pulegium L. (Lamiaceae) BCN29895

Mentha sp. (Lamiaceae)

Mentha spicata L. (Lamiaceae) BCN29995

Mentha x piperita L.
(Lamiaceae) BCN29997

Mespilus germanica L. (Rosaceae) BCN50768

Mirabilis jalapa L. (Nyctaginaceae) BCN126625

Morus alba L. (Moraceae) BCN52588

Muscari comosum (L.) Mill. (Asparagaceae) BCN29738

Myrtus communis L. (Myrtaceae) BCN35897

Amèlia, sabonera
Trèvol de ramellet
Tarongí

Tarongina

Menta d'aigua, menta de cavall

Poliol

BI

$\mathrm{BI}$

BI

Herba-sana, hierba buena (Spanish), menta

Herba-sana, menta

Menta piperita, pampalei, piperita, pipermint

Nespla (fruit), nespler, nesplera

Buenas noches (Spanish), MO, Rl dama de nit, diegos de nit, noches (Spanish), Juan de noche (Spanish), sanjoans

Morer, morera

$\mathrm{BI}$

Leaf. Stem

Calabruix

Multra, multrera, murta, murtera, murtó (fruit)

plant plant
AE Whole plant

$\mathrm{AE}, \mathrm{BI} \quad$ Aerial part.

Flower. Leaf. Stem. Whole plant $\begin{array}{ll}\mathrm{AE}, \mathrm{BI}, \mathrm{MO} & \text { Flower. Stem. } \\ & \text { Whole plant }\end{array}$

Agrosilvopastoral management: agricultural/

horticultural

Domestic: for help at home

Magic and religious beliefs and practices

Other informations: ecological information

Smoking

Magic and religious beliefs and practices

Other informations: ecological information

Agrosilvopastoral management: tools for

agricultural practices

Domestic: air freshener

Other informations: ecological information Repellent

Cosmetic

Other informations: excipient or adjuvant in medicinal or food preparations

Other informations: use not remembered

Ornamental: bouquet elaboration

Whole plant Other informations: ecological information

Leaf. Whole

Domestic: for help at home

Other informations: ecological information Repellent

Smoking

Leaf. Whole

Domestic: for help at home

Other informations: ecological information

Fruit. Stem. Whole plant

Folk oral literature

Other informations: ecological information Timber

Ornamental: gardening

Other informations: excipient or adjuvant in medicinal or food preparations

Other informations: appreciations on plant or product properties

Timber: furniture manufacturing

Other informations: ecological information

Agrosilvopastoral management: tools for

agricultural practices

Agrosilvopastoral management: for fishing

Artisanal: basketry

Artisanal: tannery

Cosmetic

Folk oral literature

Magic and religious beliefs and practices

Ornamental: bouquet elaboration

Other informations: ecological information

Repellent

Whole plant

Ornamental: bouquet elaboration 
Table 1 Non-food and non-medicinal folk plant uses in the territories of Alt Empordà, Montseny and Ripollès (Catalonia), and Formentera and Mallorca (Balearic Islands) (Continued)

\begin{tabular}{ll}
\hline $\begin{array}{l}\text { Narcissus pseudonarcissus L. } \\
\text { (Amaryllidaceae) BCN47849 }\end{array}$ & Puigestela \\
$\begin{array}{l}\text { Nepeta cataria L. } \\
\text { (Lamiaceae) BCN24935 }\end{array}$ & $\begin{array}{l}\text { Herba dels moixos, nepeta, } \\
\text { nepta }\end{array}$ \\
$\begin{array}{l}\text { Nerium oleander L. } \\
\text { (Apocynaceae) BCN46840 }\end{array}$ & Adelfa (Spanish), baladre \\
& \\
$\begin{array}{l}\text { Nicotiana glauca Graham } \\
\text { (Solanaceae) BCN81462 }\end{array}$ & $\begin{array}{l}\text { Tabac fulla d'espasa } \\
\text { Nicotiana rustica L. } \\
\text { (Solanaceae) BCN46839 }\end{array}$ \\
$\begin{array}{l}\text { Nicotiana tabacum L. } \\
\text { (Solanaceae) BCN48711 }\end{array}$ & $\begin{array}{l}\text { Tabac de pota, tabac pota } \\
\text { product), tabac, tabac pota, } \\
\text { tabaquera } \\
\text { Ocimum basilicum L. } \\
\text { (Lamiaceae) BCN29897 }\end{array}$ \\
$\begin{array}{l}\text { Alfabaguera, alfàbrega, } \\
\text { aufabeguera, albahaca } \\
\text { (Spanish), enflàgam }\end{array}$
\end{tabular}

Rl Aerial part

$\mathrm{Bl}, \mathrm{MO}, \mathrm{Rl}$ Aerial part. Whole plant

AE, BI, MO Leaf. Stem. Whole plant

$\mathrm{BI}$

$\mathrm{BI}$

AE, BI, MO Leaf. Whole plant

$\mathrm{AE}, \mathrm{BI}, \mathrm{RI} \quad$ Aerial part. Leaf. Whole plant
Ornamental: bouquet elaboration

Ornamental: gardening

Agrosilvopastoral management: tools for agricultural practices Folk oral literature

Agrosilvopastoral management: tools for agricultural practices

Artisanal: musical instrument elaboration Magic and religious beliefs and practices Ornamental: gardening

Repellent

Smoking

Leaf. Whole Other informations: ecological information plant Repellent

Smoking

Domestic: for help at home

Other informations: purchased in commerce

Repellent

Smoking

Agrosilvopastoral management: tools for agricultural practices

Cosmetic

Domestic: air freshener

Ludic

Magic and religious beliefs and practices

Ornamental: gardening

Other informations: ecological information Repellent

Odontites lutea (L.) Clairv. (Orobanchaceae) BCN73750

Olea europaea L. subsp. europaea (Oleaceae) BCN29898
Brancadella

MO Flower
Oli d'oliva (elaborated AE, Bl, Aerial part. product), oliva (fruit), olivera $\mathrm{MO}, \mathrm{RI} \quad$ Fruit. Fruit

juice. Not reported. Seed fruit. Stem. Whole plant
Agrosilvopastoral management: tools for agricultural practices

Artisanal: basketry

Artisanal: cane elaboration

Cosmetic

Domestic: for help at home

Folk oral literature

Fuel obtaining: firewood

Fuel obtaining: plant oil

Ludic

Magic and religious beliefs and practices

Ornamental: bouquet elaboration

Other informations: ecological information

Other informations: excipient or adjuvant in medicinal or food preparations

Timber

Timber: boat manufacturing

Timber: furniture manufacturing

Engís, olivera, olivera borda, $\mathrm{AE}, \mathrm{BI} \quad$ Aerial part. ullastre

Agrosilvopastoral management: agricultural/
Leaf. Stem. Whole plant horticultural

Agrosilvopastoral management: for fishing Agrosilvopastoral management: for hunting Artisanal: basketry

Artisanal: cane elaboration

Artisanal: musical instrument elaboration

Domestic: for help at home

Fuel obtaining: charcoal

Fuel obtaining: firewood

Ludic

Magic and religious beliefs and practices Other informations: appreciations on plant or product properties 
Table 1 Non-food and non-medicinal folk plant uses in the territories of Alt Empordà, Montseny and Ripollès (Catalonia), and Formentera and Mallorca (Balearic Islands) (Continued)

\begin{tabular}{|c|c|c|c|c|c|}
\hline & & & & $\begin{array}{l}\text { Timber } \\
\text { Timber: wheeled vehicles manufacturing } \\
\text { Timber: furniture manufacturing }\end{array}$ & \\
\hline $\begin{array}{l}\text { Onobrychis supina (Vill.) DC. } \\
\text { (Fabaceae) BCN52589 }\end{array}$ & Trepadella, trepadella borda & $\mathrm{MO}$ & Flower & $\begin{array}{l}\text { Agrosilvopastoral management: agricultural/ } \\
\text { horticultural }\end{array}$ & 1 \\
\hline $\begin{array}{l}\text { Onobrychis viciifolia Scop. } \\
\text { (Fabaceae) BCN26745 }\end{array}$ & Esparceta, trepadella & $\mathrm{MO}$ & Flower & $\begin{array}{l}\text { Agrosilvopastoral management: agricultural/ } \\
\text { horticultural }\end{array}$ & 1 \\
\hline $\begin{array}{l}\text { Ononis spinosa } \mathrm{L} \text {. } \\
\text { (Fabaceae) BCN50770 }\end{array}$ & Gavó & $\mathrm{AE}$ & Stem & Fuel obtaining: firewood & 1 \\
\hline $\begin{array}{l}\text { Ononis spinosa L. subsp. } \\
\text { antiquorum (L.) Briq. } \\
\text { (Fabaceae) BCN62318 }\end{array}$ & Augó, ugó & $\mathrm{Bl}$ & Fruit & Other informations: undesirable actions & 1 \\
\hline $\begin{array}{l}\text { Ophrys speculum Link } \\
\text { (Orchidaceae) BCN533 }\end{array}$ & $\begin{array}{l}\text { Orquídia, sabateta de bon } \\
\text { Jesús }\end{array}$ & $\mathrm{Bl}$ & Aerial part & $\begin{array}{l}\text { Ludic } \\
\text { Ornamental: bouquet elaborations }\end{array}$ & 2 \\
\hline $\begin{array}{l}\text { Opuntia maxima Mill. } \\
\text { (Cactaceae) BCN46078 }\end{array}$ & $\begin{array}{l}\text { Fulla de figa de moro } \\
\text { (cladode), fulla de moro, } \\
\text { fulla de moro (cladode), } \\
\text { fulla de figuera de moro } \\
\text { (cladode), figa de moro } \\
\text { (fruit), figuera de moro, } \\
\text { figuera de pic }\end{array}$ & $\mathrm{Bl}$ & $\begin{array}{l}\text { Cladode. Fruit. } \\
\text { Leaf. Leaf juice. } \\
\text { Whole plant, }\end{array}$ & $\begin{array}{l}\text { Agrosilvopastoral management: tools for } \\
\text { agricultural practices } \\
\text { Artisanal: cookware elaboration } \\
\text { Cosmetic } \\
\text { Domestic: for help at home } \\
\text { Ludic } \\
\text { Other informations: excipient or adjuvant in } \\
\text { medicinal or food preparations }\end{array}$ & 19 \\
\hline $\begin{array}{l}\text { Origanum majorana L. } \\
\text { (Lamiaceae) BCN95545 }\end{array}$ & $\begin{array}{l}\text { Marduix, morduix, } \\
\text { morduixera }\end{array}$ & $\mathrm{Bl}, \mathrm{MO}, \mathrm{Rl}$ & $\begin{array}{l}\text { Aerial part. } \\
\text { Leaf. Whole } \\
\text { plant }\end{array}$ & $\begin{array}{l}\text { Agrosilvopastoral management: agricultural/ } \\
\text { horticultural } \\
\text { Cosmetic } \\
\text { Folk oral literature } \\
\text { Other informations: ecological information }\end{array}$ & 8 \\
\hline $\begin{array}{l}\text { Origanum vulgare L. } \\
\text { (Lamiaceae) BCN24938 }\end{array}$ & Orenga, clements & $\mathrm{AE}, \mathrm{Bl}, \mathrm{Rl}$ & $\begin{array}{l}\text { Aerial part. } \\
\text { Flower. Whole } \\
\text { plant }\end{array}$ & $\begin{array}{l}\text { Domestic: air freshener } \\
\text { Domestic: for help at home } \\
\text { Folk oral literature } \\
\text { Other informations: harvesting and/or selling } \\
\text { Repellent }\end{array}$ & 16 \\
\hline $\begin{array}{l}\text { Orobanche crenata Forsk. } \\
\text { (Orobanchaceae) BCN29743 }\end{array}$ & Frare & $\mathrm{AE}$ & Whole plant & Other informations: ecological information & 1 \\
\hline $\begin{array}{l}\text { Oryza sativa L. (Poaceae) } \\
\text { BCN30000 }\end{array}$ & Arròs & $\mathrm{AE}, \mathrm{BI}, \mathrm{MO}$ & $\begin{array}{l}\text { Bran. Not } \\
\text { reported. fruit. } \\
\text { Stem }\end{array}$ & $\begin{array}{l}\text { Agrosilvopastoral management: agricultural/ } \\
\text { horticultural } \\
\text { Domestic: for help at home } \\
\text { Magic and religious beliefs and practices } \\
\text { Other informations: excipient or adjuvant in } \\
\text { medicinal or food preparations }\end{array}$ & 4 \\
\hline $\begin{array}{l}\text { Oryzopsis miliacea (L.) Asch. } \\
\text { \& Graebn. (Poaceae) } \\
\text { BCN52590 }\end{array}$ & $\begin{array}{l}\text { Fenal no vertader, herba } \\
\text { prima }\end{array}$ & $\mathrm{MO}$ & Aerial part & Ornamental: bouquet elaboration & 2 \\
\hline $\begin{array}{l}\text { Osyris alba L. (Santalaceae) } \\
\text { BCN100425 }\end{array}$ & Assot, ginestra & $\mathrm{Bl}$ & Aerial part & Other informations: ecological information & 1 \\
\hline $\begin{array}{l}\text { Oxalis articulata Savigny } \\
\text { (Oxalidaceae) BCN31290 }\end{array}$ & Trèvol & $\mathrm{AE}$ & $\begin{array}{l}\text { Leaf. Whole } \\
\text { plant }\end{array}$ & $\begin{array}{l}\text { Magic and religious beliefs and practices } \\
\text { Other informations: ecological information }\end{array}$ & 5 \\
\hline $\begin{array}{l}\text { Oxalis pes-caprae } \mathrm{L} \text {. } \\
\text { (Oxalidaceae) BCN95553 }\end{array}$ & Vinagrella, trèvol de jardí & $\mathrm{Bl}$ & Whole plant & $\begin{array}{l}\text { Agrosilvopastoral management: weeds } \\
\text { Folk oral literature } \\
\text { Other informations: ecological information }\end{array}$ & 5 \\
\hline $\begin{array}{l}\text { Paeonia mascula (L.) Mill. } \\
\text { subsp. cambessedesii (Willk.) } \\
\text { O.Bolòs \& Vigo } \\
\text { (Paeoniaceae) BCN64801 }\end{array}$ & Paloni de boca, peònia & $\mathrm{Bl}$ & Whole plant & $\begin{array}{l}\text { Folk oral literature } \\
\text { Ornamental: gardening }\end{array}$ & 3 \\
\hline $\begin{array}{l}\text { Paliurus spina-christi Mill. } \\
\text { (Rhamnaceae) BCN29902 }\end{array}$ & Espinavessa & $\mathrm{AE}$ & Root. Stem & $\begin{array}{l}\text { Artisanal: cane elaboration } \\
\text { Domestic: for help at home } \\
\text { Magic and religious beliefs and practices }\end{array}$ & 6 \\
\hline $\begin{array}{l}\text { Pancratium maritimum L. } \\
\text { (Amaryllidaceae) BCN13292 }\end{array}$ & Lliri & $\mathrm{AE}$ & Aerial part & Ornamental: bouquet elaboration & 2 \\
\hline
\end{tabular}


Table 1 Non-food and non-medicinal folk plant uses in the territories of Alt Empordà, Montseny and Ripollès (Catalonia), and Formentera and Mallorca (Balearic Islands) (Continued)

Papaver rhoeas L.
(Papaveraceae) BCN29903

Papaver somniferum L. (Papaveraceae) BCN24941

Papaver somniferum L. subsp. setigerum (DC.) Arcang. (Papaveraceae) BCN46071

Papaver somniferum L. subsp. somniferum (Papaveraceae) BCN126627

Paradisea liliastrum (L.) Bertol. (Liliaceae) BCN27270

Parietaria officinalis L. subsp. Cama-roja, herba mollera, judaica (L.) Bég. (Urticaceae) molla roquera, mollera, BCN29745

Passiflora caerulea L. (Passifloraceae) BCN29747

Pelargonium capitatum (L.) L'Hér. (Geraninaceae) BCN72719

\section{Pelargonium sp. (Geraniaceae).}

Petroselinum crispum (Mill.) Julivert Fuss (Apiaceae) BCN29905

Phagnalon saxatile (L.) Cass. (Asteraceae) BCN95555

Phaseolus vulgaris L. (Fabaceae) BCN46837

Philadelphus coronarius L. (Hydrangeaceae) BCN27261

Phillyrea angustifolia L. (Oleaceae) BCN126631

Phlomis italica $\mathrm{L}$. (Lamiaceae) BCN64879

Phoenix canariensis Chabaud (Arecaceae) BCN23835

ascall, cascai, dormidera (Spanish), roella

Cascall, dormidera

Lliri blanc morella, morella roquera, morera roquera, roca mollera, parietària

Gerani d'olor, vauma rosa

Gerani

Herba de capxerigany

Mongeta, mongeta seca

Xeringuilla, xeringuillo

Mat, mata

Estepa blenera paumera

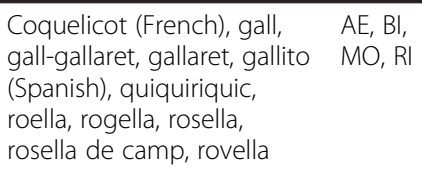

Coquelicot (French), gall, $\quad \mathrm{AE}, \mathrm{BI}$ gall-gallaret, gallaret, gallito $\mathrm{MO}, \mathrm{RI}$ (Spanish), quiquiriquic, roella, rogella, rosella, rosella de camp, rovella

Cascall, dormidera (Spanish) BI, RI (Spanish), herba queixalera

$\mathrm{AE}, \mathrm{BI}, \mathrm{MO}$ Aerial part. Leaf. Not reported. Stem. Magic and religious beliefs and practices Whole plant Other informations: ecological information

Claus de l'infern, passiflora B

Aerial part

Aerial part. Flower. Whole plant

$\mathrm{AE}, \mathrm{BI} \mathrm{RI}$ Aerial part. Leaf. Stem. Whole plant

$\mathrm{BI}$

Whole plant

$A E, B I \quad$ Seed. Whole plant

$\mathrm{Bl}, \mathrm{MO}, \mathrm{RI}$ Aerial part. Flower. Whole plant

Stem

$\mathrm{MO}$

B

Leaf. Not reported

Fasser, dàtil (fruit), palmera, BI
Leaf. Whole plant Other informations: use not remembered

Cosmetic

Ludic

Magic and religious beliefs and practices

Ornamental: bouquet elaboration

Other informations: ecological information

Ornamental: bouquet elaboration

Other informations: ecological information Other informations: appreciations on plant or product properties

Ornamental: bouquet elaboration

Other informations: ecological information

Ornamental: gardening

Agrosilvopastoral management: tools for Ornamental: gardening Other informations: ecological information Repellent

Domestic: air freshener

Other informations: ecological information

Agrosilvopastoral management: agricultural/ horticultural

Cosmetic

Folk oral literature

Other informations: ecological information

Other informations

Agrosilvopastoral management: agricultural/

Ludic

Magic and religious beliefs and practices

Ornamental: bouquet elaboration

Ornamental: gardening

Timber: construction materials production

Agrosilvopastoral management: tools for agricultural practices

Cosmetic

Domestic: for help at home Ornamental: bouquet elaboration

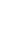

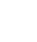

(1)


Table 1 Non-food and non-medicinal folk plant uses in the territories of Alt Empordà, Montseny and Ripollès (Catalonia), and Formentera and Mallorca (Balearic Islands) (Continued)

(Arecaceae) BCN51783
Phoenix dactylifera L.
Fasser, dàtil (fruit), palmera, Bl, MO pauma, paumera

\section{Phragmites australis (Cav.) Trin. ex Steud. (Poaceae) BCN88033 \\ Phyllitis scolopendrium (L.) Newman (Aspleniaceae) BCN29906 \\ Phyllostachys aurea Rivière \& C.Rivière (Poaceae) BCN30002}

Phyllostachys flexuosa BCN30001

Physalis alkekengi L. (Solanaceae) BCN31293

Phytolacca americana L. (Phytolaccaceae) BCN81961

Picea abies (L.) H.Karst. (Pinaceae) BCN36717

Picris hieracioides Sibth. \& Sm. (Asteraceae) BCN4916

Pimpinella anisum L. (Apiaceae) BCN47278

Pinus halepensis Mill. (Pinaceae) BCN29826 Rivière \& C.Rivière (Poaceae)
Canyet, canyisso

Herba melsera, melsera

Canya americana, canya de bambú, canya forastera, bambú

Canya americana negra

Alquequengi, bossa, bossa vermella, fanalet, pebro

Fitolaca

Avet, pivet

Gafallosa, pedaç

Matafaluga

Pi, pi bord, pinya (cone), reïna (resin)
$\mathrm{MO}$

Frond

AE

Stem

Aerial part.

Leaf, Thorn.

Whole plant

Not reported

$A E, B I$

Stem. Whole

plant

AE, RI, MO Aerial part.

Fruit. Whole plant

$\mathrm{MO}$

Fruit

$\mathrm{MO}$

Whole plant

MO Leaf

AE $\quad$ Fruit

AE, BI, MO Aerial part. Bark. Leaf. Not reported. Resin. Stem. Whole plant
Artisanal: basketry

Artisanal: cookware elaboration

Magic and religious beliefs and practices

Ornamental: bouquet elaboration

Other informations: ecological information

extile: fibre or cloth elaboration

Agrosilvopastoral management: tools for

Agrosilvopastoral management: for fishing Artisanal: musical instrument elaboration Other informations: ecological information

Agrosilvopastoral management: for fishing

Ornamental: bouquet elaboration

Ornamental: gardening

3

udic

2

Agrosilvopastoral management: forestry

51

Artisanal: broom elaboration

Artisanal: cookware elaboration

Artisanal: footwear elaboration

Artisanal: instrument elaboration

Artisanal: musical instrument elaboration

Artisanal: tannery

Domestic: air freshener

Domestic: for help at home

Fuel obtaining: charcoal

Fuel obtaining: firewood

Magic and religious beliefs and practices

Ornamental: bouquet elaboration

Ornamental: gardening

Other informations: ecological information

Other informations: harvesting and/or selling

Other informations: use not remembered

Textile: dyer

Timber

Timber: boat manufacturing

Timber: wheeled vehicles manufacturing

Timber: furniture manufacturing

Timber: construction materials production

Agrosilvopastoral management: honey

obtaining

Other informations: use not remembered

Timber: boat manufacturing
9

$\mathrm{AE}, \mathrm{MO} \quad$ Stem 
Table 1 Non-food and non-medicinal folk plant uses in the territories of Alt Empordà, Montseny and Ripollès (Catalonia), and Formentera and Mallorca (Balearic Islands) (Continued)

Pinus pinea L. (Pinaceae) Pi, pi de llei, pi pinyer, pi BCN26751 pinyoner, pinyó (seed)

Pinus sp. (Pinaceae)

Pinus sylvestris L. (Pinaceae) BCN46070

Piper nigrum L. (Piperaceae) BCN47277

Pistacia lentiscus L.

(Anacardiaceae) BCN29907 (elaborated product)
Pi, pinya (cone), reïna, trementina (elaborated product)

Pi, pi bord, pi rajolet, pi roig, trementina (elaborated product), pega (elaborated product), pega negra

Prebe bo, prebe bo blanc, prebe negre

Llentriscle, Ilentrisca, mat, mata, triquell

$\begin{aligned} A E, B I, M O & \text { Bark. Leaf. } \\ & \text { Seed. Stem. } \\ & \text { Whole plant }\end{aligned}$

$A E, B I, M O$ Aerial part. Bark, Flower. Leaf. Resin. Stem. Whole plant. Cortical parenchyma

AE, MO, RI Cone. Stem. Whole plant

Artisanal: footwear elaboration

Folk oral literature

Fuel obtaining: firewood

Magic and religious beliefs and practices

Ornamental: bouquet elaboration

Ornamental: gardening

Textile: dyer

Timber

Timber: boat manufacturing

Timber: wheeled vehicles manufacturing

Timber: construction materials production

Agrosilvopastoral management: forestry

Agrosilvopastoral management: honey

obtaining

Cosmetic

Domestic: for help at home

Folk oral literature

Fuel obtaining: charcoal

Fuel obtaining: firewood

Magic and religious beliefs and practices

Other informations: harvesting and/or selling

Repellent

Smoking

Textile: dyer

Artisanal: footwear elaboration

Fuel obtaining: firewood

Other informations: ecological information

Timber: boat manufacturing

Timber: construction materials production

Seed

Agrosilvopastoral management: for pig slaughter

$\mathrm{AE}, \mathrm{BI}, \mathrm{MO}$ Aerial part. Fruit juice. Leaf. Not reported.

Agrosilvopastoral management: tools for Resin. Stem.

Artisanal: basketry

Artisanal: musical instrument elaboration

Domestic: air freshener

Domestic: for help at home

Fuel obtaining: charcoal

Magic and religious beliefs and practices

Ornamental: bouquet elaboration

Other informations: use not remembered Repellent

Timber

Pistacia terebinthus $\mathrm{L}$.

Noguerola

$\mathrm{AE} \quad$ Resin

Domestic: paint elaboration

Pistacia vera $\mathrm{L}$.

Pistatxo

$\mathrm{MO}$

Whole plant

Ornamental: gardening

Whole plant

Pisum sativum L. subsp. sativum (Fabaceae)

BCN32140

Plantago afra L. (Plantaginaceae) BCN 95568

Plantago coronopus L.

subsp. coronopus

(Plantaginaceae) BCN29908

Plantago lagopus L. (Plantaginaceae) BCN98975

Plantago lanceolata L.

Estiragassó, pèsol, xítxero

BI

Agrosilvopastoral management: agricultural/ horticultural

Other informations: ecological information

Herba de puça

Aerial part.

Cosmetic

Seed

Cua de rata

AE

Leaf

Magic and religious beliefs and practices

Not reported

Other informations: ecological information

Domestic: for help at home

Ludic

llantén (Spanish), plantatge, plantatge de fulla estreta, plantatge estret, plantatge llarg, plantago
$\mathrm{Bl}, \mathrm{MO}, \mathrm{RI}$ Aerial part. Leaf. Whole plant
Other informations: ecological information

(1)


Table 1 Non-food and non-medicinal folk plant uses in the territories of Alt Empordà, Montseny and Ripollès (Catalonia), and Formentera and Mallorca (Balearic Islands) (Continued)

Plantago major L.
(Plantaginaceae) BCN24950
Platanus xhispanica Mill. ex
Münchh. (Platanaceae)
BCN31294

Plectanthus sp. (Lamiaceae)
Poinsettia pulcherrima
(Wild.) Graham
(Euphorbiaceae) BCN48713
Polygonatum odoratum
(Mill.) Druce (Liliaceae)
BCN56044
Polygonum aviculare L.
(Polygonaceae) BCN32136
Polypodium vulgare L.
(Polypodiaceae) BCN32134
Polystichum setiferum
(Forssk.) Moore ex Woyn.
(Dryopteridaceae)
BCN126634
Populus alba L. (Salicaceae)
BCN29751
PCr.

Llantén (Spanish),

plantatge, plantatge ample, plantatge rodó

Plàtan, plataner, platero

und $\begin{array}{ll}A E, B I, M O & \text { Bark. Leaf. } \\ & \text { Stem }\end{array}$

Encens, planta dels doblers

Nadala

$\mathrm{BI}$

$\mathrm{AE}$

Whole plant

Whole plant

Arracadetes

Estiravelles, triavella,

traspassacamins

Falzia, foguera borda, herba MO, RI

de les gallines, herba felera

Foguera, foguera de jardí, foguera mosquera

Arbre, arbre poll de fusta blanca, alba, arbre blanc
$\mathrm{AE}, \mathrm{MO} \quad$ Stem

Frond. Not reported

Frond
Populus nigra L. (Salicaceae) BCN29753
Arbre canadà, arbre poll de fulla fosca, arbre poll, poll, polla, pollancre, pollangre
$A E, B I, \quad$ Stem

$\mathrm{MO}, \mathrm{RI}$

Populus sp. (Salicaceae)
Populus tremula L.
(Salicaceae) BCN46836
Populus $\times$ canadensis
Moench (Salicaceae)
BCN126637
Portulaca oleracea L.

(Portulacaceae) BCN46835

Posidonia oceanica (L.) Delile (Posidoniaceae) BCN103134
Poll, pollancre

Trèmol

Poll, polla, pollancre bordil

Enciam de patena,

verdelaga, verdolaga

Alga, bolla de la mar (decomposed stem and leaf), posidònia
Aerial part. Leaf. Stem. Whole plant

Ludic

Other informations: ecological information

Agrosilvopastoral management: tools for agricultural practices

Domestic: for help at home

Magic and religious beliefs and practices Timber

Timber: boat manufacturing

Timber: furniture manufacturing

Ornamental: gardening

2

Magic and religious beliefs and practices

Ornamental: bouquet elaboration

Magic and religious beliefs and practices Ornamental: bouquet elaboration

Agrosilvopastoral management: tools for agricultural practices

Artisanal: footwear elaboration

Ludic

Timber

Agrosilvopastoral management: tools for agricultural practices

Artisanal: footwear elaboration

Domestic: for help at home

Ludic

Other informations

Timber

Timber: furniture manufacturing

Other informations: use not remembered Timber: furniture manufacturing

Artisanal: cane elaboration

Artisanal: cookware elaboration

Timber

Stem. Whole Artisanal: cookware elaboration

plant

Ornamental: gardening

Timber

Timber: construction materials production

Folk oral literature

Ornamental: gardening

Other informations: ecological information

Agrosilvopastoral management: tools for agricultural practices

Artisanal: musical instrument elaboration

Domestic: for help at home

Other informations: harvesting and/or selling

Textile: textile padding

Other informations: ecological information

Magic and religious beliefs and practices 
Table 1 Non-food and non-medicinal folk plant uses in the territories of Alt Empordà, Montseny and Ripollès (Catalonia), and Formentera and Mallorca (Balearic Islands) (Continued)

Primula veris $\mathrm{L}$.

(Primulaceae) BCN27280

Primula veris L. subsp. columnae (Ten.) Maire \& Petitm. (Primulaceae) BCN29755

Prunella grandiflora (L.) Scholler (Lamiaceae) BCN24956

Prunella grandiflora (L.) Scholler subsp. pyrenaica (Gren. \& Godr.) A. \& O.Bolòs (Lamiaceae) BCN29757

Prunella vulgaris $\mathrm{L}$. (Lamiaceae) BCN29759

Prunus armeniaca L. (Rosaceae) BCN48712

Prunus avium (L.) L. (Rosaceae) BCN29827

Prunus avium (L.) L. var. sylvestris Dierb. (Rosaceae) BCN60586

Prunus domestica L. (Rosaceae) BCN46834

Prunus dulcis (Mill.) D.A.Webb. (Rosaceae) BCN46833



Herba del traïdor, xuclaabella

Herba de la mel, xuclamel

$\mathrm{AE}$

RI Aerial part

Aerial part.

Flower

Puput

AE Aerial part

Ornamental: bouquet elaboration

1

Albercoc (fruit), albercoquer B

Bl

Endocarp. Fruit.

Stem

Anflorescence.
Whole plant

Agrosilvopastoral management: honey obtaining

Agrosilvopastoral management: honey

obtaining

Ornamental: bouquet elaboration

Other informations: ecological information

Flower Smoking

1

7

Folk oral literature

Fuel obtaining: charcoal

Fuel obtaining: firewood

Ludic

Other informations: appreciations on plant or product properties

Timber: furniture manufacturing

Cirera (fruit), cirerer

$A E, B I, M O$ Fruit. Resin.

Artisanal: basketry

Seed. Stem.

Whole plant

Artisanal: cane elaboration

Domestic: for help at home

Folk oral literature

Ludic

Other informations: appreciations on plant or

product properties

Timber

Timber: furniture manufacturing

Cirerer

$\mathrm{MO}$

Flower. Whole

plant

Agrosilvopastoral management: agricultural/

horticultural

Pruna (fruit), prunera

$\mathrm{BI}$

Aerial part.

Fruit. Whole

plant

Agrosilvopastoral management: agricultural/ horticultural

Folk oral literature

Ludic

Other informations: ecological information

Ametla (fruit), ametler, ametller

Agrosilvopastoral management: agricultural/
$A E, B I, M O$ Aerial part.

Endocarp.

Epicarp. Flower. Artisanal: musical instrument elaboration

Fruit. Fruit

juice.

Mesocarp.

Resin. Seed.

Stem. Whole

plant

Cosmetic

Domestic: for help at home

Folk oral literature

Fuel obtaining: charcoal

Fuel obtaining: firewood

Ornamental: bouquet elaboration

Other informations: use not remembered

Timber

Timber: boat manufacturing

Timber: furniture manufacturing

Melicotó (fruit),

$\mathrm{BI}, \mathrm{MO}$

Flower. Whole

plant

Agrosilvopastoral management: agricultural/

horticultural

presseguer

Other informations: ecological information

Prunus spinosa L.

Aranyoner, arç negre,

$\mathrm{BI}, \mathrm{MO}$

Stem. Whole

plant

Agrosilvopastoral management: agricultural/ horticultural

Other informations: ecological information Timber 
Table 1 Non-food and non-medicinal folk plant uses in the territories of Alt Empordà, Montseny and Ripollès (Catalonia), and Formentera and Mallorca (Balearic Islands) (Continued)

\begin{tabular}{|c|c|c|c|c|c|}
\hline $\begin{array}{l}\text { Pseudotsuga menziesii } \\
\text { (Mirb.) Franco (Pinaceae) } \\
\text { BCN36632 }\end{array}$ & Douglas, pi Douglas & $\mathrm{MO}$ & $\begin{array}{l}\text { Leaf. Whole } \\
\text { plant }\end{array}$ & $\begin{array}{l}\text { Magic and religious beliefs and practices } \\
\text { Timber }\end{array}$ & 4 \\
\hline $\begin{array}{l}\text { Psoralea bituminosa L. } \\
\text { (Fabaceae) BCN32133 }\end{array}$ & Cabrulla, calabrunya & $\mathrm{MO}$ & Flower & Magic and religious beliefs and practices & 1 \\
\hline $\begin{array}{l}\text { Pteridium aquilinum (L.) } \\
\text { Kuhn (Dennstaedtiaceae) } \\
\text { BCN46068 }\end{array}$ & $\begin{array}{l}\text { Falguera, falguera de Lluc, } \\
\text { helecho (Spanish), mata } \\
\text { falguera }\end{array}$ & $\begin{array}{l}\mathrm{AE}, \mathrm{BI} \\
\mathrm{MO}, \mathrm{RI}\end{array}$ & $\begin{array}{l}\text { Aerial part. } \\
\text { Frond. Whole } \\
\text { plant }\end{array}$ & $\begin{array}{l}\text { Agrosilvopastoral management: agricultural/ } \\
\text { horticultural } \\
\text { Domestic: for help at home } \\
\text { Folk oral literature } \\
\text { Magic and religious beliefs and practices } \\
\text { Ornamental: bouquet elaboration } \\
\text { Ornamental: gardening } \\
\text { Other informations: use not remembered }\end{array}$ & 34 \\
\hline $\begin{array}{l}\text { Punica granatum L. } \\
\text { (Lythraceae) BCN29764 }\end{array}$ & Magrana (fruit), magraner & $A E, B I, M O$ & $\begin{array}{l}\text { Flower. Fruit. } \\
\text { Stem. Whole } \\
\text { plant }\end{array}$ & $\begin{array}{l}\text { Agrosilvopastoral management: for pig } \\
\text { slaughter } \\
\text { Artisanal: cane elaboration } \\
\text { Artisanal: cookware elaboration } \\
\text { Artisanal: musical instrument elaboration } \\
\text { Domestic: for help at home } \\
\text { Folk oral literature } \\
\text { Ludic } \\
\text { Magic and religious beliefs and practices } \\
\text { Ornamental: bouquet elaboration } \\
\text { Ornamental: gardening } \\
\text { Other informations: ecological information } \\
\text { Textile: dyer }\end{array}$ & 16 \\
\hline $\begin{array}{l}\text { Pyracantha coccinea } \\
\text { M.Roem. (Rosaceae) } \\
\text { BCN52591 }\end{array}$ & Cartegus & $\mathrm{MO}$ & Whole plant & Ornamental: gardening & 1 \\
\hline $\begin{array}{l}\text { Pyrus communis L. subsp. } \\
\text { communis (Rosaceae) } \\
\text { BCN46831 }\end{array}$ & Pera (fruit), perera & $\mathrm{AE}, \mathrm{Bl}, \mathrm{MO}$ & $\begin{array}{l}\text { Flower. Stem. } \\
\text { Whole plant }\end{array}$ & $\begin{array}{l}\text { Agrosilvopastoral management: agricultural/ } \\
\text { horticultural } \\
\text { Artisanal: musical instrument elaboration } \\
\text { Other informations: ecological information }\end{array}$ & 3 \\
\hline $\begin{array}{l}\text { Pyrus malus L. subsp. } \\
\text { malus (Rosaceae) } \\
\text { BCN24961 }\end{array}$ & Pomera borda & $\mathrm{Rl}$ & Aerial part & $\begin{array}{l}\text { Agrosilvopastoral management: agricultural/ } \\
\text { horticultural }\end{array}$ & 1 \\
\hline $\begin{array}{l}\text { Pyrus malus L. subsp. mitis } \\
\text { (Wallr.) O.Bolòs \& J.Vigo } \\
\text { (Rosaceae) BCN46830 }\end{array}$ & $\begin{array}{l}\text { Poma (fruit), poma bellesa } \\
\text { de Roma (fruit), poma de } \\
\text { bosc (fruit), poma del ciri } \\
\text { (fruit), poma estarqui (fruit), } \\
\text { poma fadrineta (fruit), } \\
\text { poma goldén (fruit), poma } \\
\text { manyaga (fruit), poma } \\
\text { reneta (fruit), pomera, } \\
\text { pomera borda, vinagre de } \\
\text { poma (elaborated product) }\end{array}$ & $\mathrm{AE}, \mathrm{BI}, \mathrm{MO}$ & $\begin{array}{l}\text { Fruit. Whole } \\
\text { plant }\end{array}$ & $\begin{array}{l}\text { Agrosilvopastoral management: agricultural/ } \\
\text { horticultural } \\
\text { Artisanal: cane elaboration } \\
\text { Domestic: air freshener } \\
\text { Other informations: ecological information }\end{array}$ & 8 \\
\hline $\begin{array}{l}\text { Quercus alba L. (Fagaceae) } \\
\text { BCN60896 }\end{array}$ & Roure americà & $A E$ & Stem & Domestic: for help at home & 1 \\
\hline $\begin{array}{l}\text { Quercus coccifera L. subsp. } \\
\text { coccifera (Fagaceae) } \\
\text { BCN29765 }\end{array}$ & Garric, garriga, garrolla & $\mathrm{AE}$ & $\begin{array}{l}\text { Stem. Whole } \\
\text { plant }\end{array}$ & $\begin{array}{l}\text { Artisanal: broom elaboration } \\
\text { Other informations: ecological information }\end{array}$ & 2 \\
\hline $\begin{array}{l}\text { Quercus humilis Mill. } \\
\text { (Fagaceae) BCN32132 }\end{array}$ & Roure & $\mathrm{MO}$ & $\begin{array}{l}\text { Gall. Stem. } \\
\text { Whole plant }\end{array}$ & $\begin{array}{l}\text { Fuel obtaining: charcoal } \\
\text { Ludic } \\
\text { Timber }\end{array}$ & 4 \\
\hline $\begin{array}{l}\text { Quercus ilex L. (Fagaceae) } \\
\text { BCN24963 }\end{array}$ & Aglà (fruit), alzina, aulina & $\mathrm{Bl}, \mathrm{RI}$ & $\begin{array}{l}\text { Bark. Flower. } \\
\text { Leaf. Stem. } \\
\text { Whole plant }\end{array}$ & $\begin{array}{l}\text { Agrosilvopastoral management: honey } \\
\text { obtaining } \\
\text { Agrosilvopastoral management: tools for } \\
\text { agricultural practices } \\
\text { Artisanal }\end{array}$ & 31 \\
\hline
\end{tabular}


Table 1 Non-food and non-medicinal folk plant uses in the territories of Alt Empordà, Montseny and Ripollès (Catalonia), and Formentera and Mallorca (Balearic Islands) (Continued)

Quercus ilex L. subsp. ilex (Fagaceae) BCN29932

Quercus petraea (Matt.) Liebl. (Fagaceae) BCN29829
Aglà (fruit), alzina, roure

Aglà (fruit), alzina, roure, roure francès, roure hongarès
$A E, M O, R I$ Fruit. Gall. Stem. Whole plant. Cortical parenchyma

AE Stem

$\mathrm{AE}, \mathrm{MO} \quad$ Bark

Artisanal: tannery

Cosmetic

Folk oral literature

Fuel obtaining: firewood

Ludic

Magic and religious beliefs and practices

Ornamental: gardening

Other informations: ecological information

Other informations: use not remembered

Textile: dyer

Timber

Timber: boat manufacturing

Timber: furniture manufacturing

Agrosilvopastoral management: tools for agricultural practices

Agrosilvopastoral management: for pig

slaughter

Artisanal: broom elaboration

Artisanal: cane elaboration

Artisanal: cookware elaboration

Domestic: for help at home

Folk oral literature

Fuel obtaining: charcoal

Fuel obtaining: firewood

Ludic

Magic and religious beliefs and practices

Ornamental: gardening

Timber: boat manufacturing

Timber: wheeled vehicles manufacturing

Agrosilvopastoral management: tools for agricultural practices

Fuel obtaining: charcoal

Fuel obtaining: firewood

Ludic

Other informations: ecological information

Timber: boat manufacturing

Timber: furniture manufacturing

Timber: construction materials production

Quercus pubescens Willd.

(Fagaceae) BCN30007

Quercus suber L. (Fagaceae) Alzina surera, suro BCN46829

Roure

(n)

\section{Ranunculus asiaticus L. \\ (Ranunculaceae) BCN65283 \\ Francesilla}

Raphanus raphanistrum L. (Brassicaceae) BCN30042

Raphia sp. (Arecaceae)

Rhamnus alaternus L. (Rhamnaceae) BCN29769
Ravenissa

Ràfia

Aladern, llampúdol, llampudoler, mata
Agrosilvopastoral management: tools for agricultural practices

Agrosilvopastoral management: tools for agricultural practices

Agrosilvopastoral management: for fishing Domestic: for help at home

Folk oral literature

Ludic

Other informations: use not remembered

Textile: dyer

Textile: textile padding

Ornamental: bouquet elaboration

Ornamental: gardening

Bl Whole plant

Agrosilvopastoral management: agricultural/ horticultural

Artisanal: basketry

Artisanal: broom elaboration

Artisanal: cookware elaboration

Artisanal: smoking pipe elaboration

Fuel obtaining: charcoal

Ornamental: bouquet elaboration

Ornamental: gardening

Timber

Timber: furniture manufacturing Stem. Whole plant 
Table 1 Non-food and non-medicinal folk plant uses in the territories of Alt Empordà, Montseny and Ripollès (Catalonia), and Formentera and Mallorca (Balearic Islands) (Continued)

\begin{tabular}{|c|c|c|c|c|c|}
\hline $\begin{array}{l}\text { Rhamnus ludovici-salvatoris } \\
\text { Chodat (Rhamnaceae) } \\
\text { BCN30057 }\end{array}$ & Aladern, Ilampúdol & $\mathrm{Bl}$ & Whole plant & Ornamental: gardening & 1 \\
\hline $\begin{array}{l}\text { Rhododendron ferrugineum } \\
\text { L. (Ericaceae) BCN24969 }\end{array}$ & Gafet, neret & $\mathrm{Rl}$ & $\begin{array}{l}\text { Aerial part. } \\
\text { Whole plant }\end{array}$ & $\begin{array}{l}\text { Ornamental: bouquet elaboration } \\
\text { Other informations: ecological information }\end{array}$ & 3 \\
\hline $\begin{array}{l}\text { Ricinus communis L. } \\
\text { (Euphorbiaceae) BCN46089 }\end{array}$ & $\begin{array}{l}\text { Cagamutxo, oli de ricí } \\
\text { (elaborated product), recino } \\
\text { (Spanish), resiner, ricino } \\
\text { (Spanish) }\end{array}$ & $\mathrm{AE}, \mathrm{BI}$ & Seed. Seed oil & $\begin{array}{l}\text { Magic and religious beliefs and practices } \\
\text { Other informations: purchased in commerce }\end{array}$ & 13 \\
\hline $\begin{array}{l}\text { Robinia pseudoacacia L. } \\
\text { (Fabaceae) BCN31298 }\end{array}$ & Acàcia, escàcia & $\begin{array}{l}\mathrm{AE}, \mathrm{BI} \\
\mathrm{MO}, \mathrm{RI}\end{array}$ & $\begin{array}{l}\text { Flower. Not } \\
\text { reported. Stem }\end{array}$ & $\begin{array}{l}\text { Agrosilvopastoral management: tools for } \\
\text { agricultural practices } \\
\text { Domestic: for help at home } \\
\text { Magic and religious beliefs and practices } \\
\text { Timber: boat manufacturing } \\
\text { Timber: wheeled vehicles manufacturing } \\
\text { Timber: furniture manufacturing } \\
\text { Timber: construction materials production }\end{array}$ & 11 \\
\hline $\begin{array}{l}\text { Rorippa nasturtium- } \\
\text { aquaticum (L.) Hyek } \\
\text { (Brassicaceae) BCN29771 }\end{array}$ & $\begin{array}{l}\text { Créixec, creixen, créixol, } \\
\text { créssec }\end{array}$ & $\mathrm{Bl}$ & Aerial part & $\begin{array}{l}\text { Other informations: appreciations on plant or } \\
\text { product properties }\end{array}$ & 2 \\
\hline $\begin{array}{l}\text { Rosa alba L. (Rosaceae) } \\
\text { BCN400041 }\end{array}$ & Roser blanc & $\mathrm{Bl}$ & Whole plant & Ornamental: gardening & 1 \\
\hline $\begin{array}{l}\text { Rosa canina L. (Rosaceae) } \\
\text { BCN29772 }\end{array}$ & $\begin{array}{l}\text { Gavarrera, roser bord, roser } \\
\text { de bosc, roser de pastor, } \\
\text { roser silvestre }\end{array}$ & $A E, M O$ & $\begin{array}{l}\text { Aerial part. } \\
\text { Fruit. Stem. } \\
\text { Whole plant }\end{array}$ & $\begin{array}{l}\text { Agrosilvopastoral management: tools for } \\
\text { agricultural practices } \\
\text { Artisanal: smoking pipe elaboration } \\
\text { Ludic } \\
\text { Ornamental: bouquet elaboration }\end{array}$ & 6 \\
\hline $\begin{array}{l}\text { Rosa gallica L. (Rosaceae) } \\
\text { BCN24972 }\end{array}$ & Roser, roser de marge & $\mathrm{MO}, \mathrm{RI}$ & $\begin{array}{l}\text { Flower. Whole } \\
\text { plant }\end{array}$ & $\begin{array}{l}\text { Folk oral literature } \\
\text { Magic and religious beliefs and practices } \\
\text { Ornamental: bouquet elaboration }\end{array}$ & 7 \\
\hline Rosa sp. (Rosaceae) & Roser, roser de jardí & $\begin{array}{l}\mathrm{AE}, \mathrm{BI} \\
\mathrm{MO}, \mathrm{RI}\end{array}$ & $\begin{array}{l}\text { Corolla. Flower. } \\
\text { Leaf. Whole } \\
\text { plant }\end{array}$ & $\begin{array}{l}\text { Agrosilvopastoral management: tools for } \\
\text { agricultural practices } \\
\text { Cosmetic } \\
\text { Domestic: air freshener } \\
\text { Folk oral literature } \\
\text { Magic and religious beliefs and practices } \\
\text { Ornamental: bouquet elaboration } \\
\text { Ornamental: gardening } \\
\text { Other informations: ecological informations }\end{array}$ & 24 \\
\hline $\begin{array}{l}\text { Rosa tomentosa Sm. } \\
\text { (Rosaceae) BCN24973 }\end{array}$ & $\begin{array}{l}\text { Grataculs, roser, roser bord, } \\
\text { roser de bosc }\end{array}$ & $\mathrm{Rl}$ & $\begin{array}{l}\text { Fruit. Whole } \\
\text { plant }\end{array}$ & $\begin{array}{l}\text { Ludic } \\
\text { Ornamental: gardening }\end{array}$ & 2 \\
\hline $\begin{array}{l}\text { Rosa centifolia L. } \\
\text { (Rosaceae) BCN126648 }\end{array}$ & Roser, roser de marge & $\mathrm{MO}$ & Flower & $\begin{array}{l}\text { Magic and religious beliefs and practices } \\
\text { Other informations }\end{array}$ & 2 \\
\hline $\begin{array}{l}\text { Rosmarinus officinalis L. } \\
\text { (Lamiaceae) BCN29937 }\end{array}$ & Romaní & $\begin{array}{l}\mathrm{AE}, \mathrm{BI} \\
\mathrm{MO}, \mathrm{RI}\end{array}$ & $\begin{array}{l}\text { Aerial part. } \\
\text { Flower. Stem. } \\
\text { Whole plant }\end{array}$ & $\begin{array}{l}\text { Agrosilvopastoral management: weeds } \\
\text { Cosmetic } \\
\text { Domestic: air freshener } \\
\text { Folk oral literature } \\
\text { Magic and religious beliefs and practices } \\
\text { Other informations: ecological information } \\
\text { Repellent }\end{array}$ & 43 \\
\hline $\begin{array}{l}\text { Rubus ulmifolius Schott } \\
\text { (Rosaceae) BCN32130 }\end{array}$ & $\begin{array}{l}\text { Barder, batzer, móra (fruit), } \\
\text { romeguer, romeguera }\end{array}$ & $\mathrm{Bl}, \mathrm{MO}$ & $\begin{array}{l}\text { Flower. Whole } \\
\text { plant. Young } \\
\text { shoot }\end{array}$ & $\begin{array}{l}\text { Agrosilvopastoral management: honey } \\
\text { obtaining } \\
\text { Folk oral literature } \\
\text { Other informations: ecological information }\end{array}$ & 10 \\
\hline $\begin{array}{l}\text { Rumex acetosa L. subsp. } \\
\text { acetosa (Polygonaceae) } \\
\text { BCN29775 }\end{array}$ & Llengua de bou & $A E$ & Aerial part & Ornamental: bouquet elaboration & 1 \\
\hline $\begin{array}{l}\text { Rumex crispus L. } \\
\text { (Polygonaceae) } \\
\text { BCN126650 }\end{array}$ & $\begin{array}{l}\text { Cama-roja, paradella, Santa } \\
\text { Maria, vinagrella }\end{array}$ & $\mathrm{MO}$ & Fruit & Magic and religious beliefs and practices & 3 \\
\hline
\end{tabular}


Table 1 Non-food and non-medicinal folk plant uses in the territories of Alt Empordà, Montseny and Ripollès (Catalonia), and Formentera and Mallorca (Balearic Islands) (Continued)

\begin{tabular}{|c|c|c|c|c|c|}
\hline Rumex sp. (Polygonaceae) & Llengua de bou & $\mathrm{Rl}$ & Leaf & Ludic & 1 \\
\hline $\begin{array}{l}\text { Ruscus aculeatus L. } \\
\text { (Asparagaceae) BCN29939 }\end{array}$ & $\begin{array}{l}\text { Bolleta del bon pastor, } \\
\text { bolleta de Nadal, boix marí, } \\
\text { cirerer de Betlem, gallaranc, } \\
\text { galzeran }\end{array}$ & $\begin{array}{l}\mathrm{AE}, \mathrm{BI} \\
\mathrm{MO}, \mathrm{RI}\end{array}$ & $\begin{array}{l}\text { Aerial part. } \\
\text { Stem. Whole } \\
\text { plant }\end{array}$ & $\begin{array}{l}\text { Agrosilvopastoral management: for fishing } \\
\text { Magic and religious beliefs and practices } \\
\text { Ornamental: bouquet elaboration } \\
\text { Ornamental: gardening } \\
\text { Other informations: use not remembered }\end{array}$ & 20 \\
\hline $\begin{array}{l}\text { Ruta chalepensis L. } \\
\text { (Rutaceae) BCN29940 }\end{array}$ & $\begin{array}{l}\text { Abrecaminos (Spanish), } \\
\text { ruda }\end{array}$ & $\mathrm{AE}, \mathrm{BI}, \mathrm{Rl}$ & $\begin{array}{l}\text { Aerial part. Not } \\
\text { reported. Stem. } \\
\text { Whole plant }\end{array}$ & $\begin{array}{l}\text { Agrosilvopastoral management: tools for } \\
\text { agricultural practices } \\
\text { Cosmetic } \\
\text { Domestic: air freshener } \\
\text { Folk oral literature } \\
\text { Magic and religious beliefs and practices } \\
\text { Ornamental: bouquet elaboration } \\
\text { Other informations: ecological information } \\
\text { Other informations: appreciations on plant or } \\
\text { product properties } \\
\text { Repellent }\end{array}$ & 37 \\
\hline $\begin{array}{l}\text { Ruta chalepensis L. subsp. } \\
\text { chalepensis (Rutaceae) } \\
\text { BCN32128 }\end{array}$ & Ruda & $\mathrm{MO}$ & Whole plant & Folk oral literature & 6 \\
\hline $\begin{array}{l}\text { Ruta graveolens L. } \\
\text { (Rutaceae) BCN29776 }\end{array}$ & Ruda & $\mathrm{Bl}$ & Aerial part & Magic and religious beliefs and practices & 5 \\
\hline $\begin{array}{l}\text { Saccharum officinarum L. } \\
\text { (Poaceae) BCN50771 }\end{array}$ & $\begin{array}{l}\text { Canya de sucre, canyamel, } \\
\text { melassa (elaborated } \\
\text { product) }\end{array}$ & $\mathrm{Bl}$ & Stem & $\begin{array}{l}\text { Agrosilvopastoral management: tools for } \\
\text { agricultural practices }\end{array}$ & 1 \\
\hline $\begin{array}{l}\text { Salix alba L. (Salicaceae) } \\
\text { BCN29777 }\end{array}$ & Saula, saule & $A E, R I$ & Stem & $\begin{array}{l}\text { Artisanal: broom elaboration } \\
\text { Artisanal: footwear elaboration } \\
\text { Agrosilvopastoral management: for fishing } \\
\text { Ornamental: bouquet elaboration } \\
\text { Other informations: ecological information }\end{array}$ & 6 \\
\hline $\begin{array}{l}\text { Salix alba L. subsp. alba } \\
\text { (Salicaceae) BCN56012 }\end{array}$ & Saula, saule, sàlic & $\mathrm{MO}, \mathrm{RI}$ & Flower. Stem & $\begin{array}{l}\text { Agrosilvopastoral management: tools for } \\
\text { agricultural practices } \\
\text { Artisanal: footwear elaboration } \\
\text { Timber: wheeled vehicles manufacturing }\end{array}$ & 8 \\
\hline $\begin{array}{l}\text { Salix atrocinerea Brot. } \\
\text { (Salicaceae) BCN39996 }\end{array}$ & Gatell & $\mathrm{AE}$ & Whole plant & Other informations: ecological information & 1 \\
\hline $\begin{array}{l}\text { Salix babylonica L. } \\
\text { (Salicaceae) BCN50772 }\end{array}$ & Desmai & $\mathrm{AE}, \mathrm{MO}$ & $\begin{array}{l}\text { Stem. Whole } \\
\text { plant }\end{array}$ & $\begin{array}{l}\text { Artisanal: basketry } \\
\text { Ornamental: gardening }\end{array}$ & 4 \\
\hline $\begin{array}{l}\text { Salix caprea L. (Salicaceae) } \\
\text { BCN46073 }\end{array}$ & $\begin{array}{l}\text { Gat saule, gatells, salze, } \\
\text { saule }\end{array}$ & $A E, R I$ & $\begin{array}{l}\text { Aerial part. } \\
\text { Stem }\end{array}$ & Ornamental: bouquet elaboration & 6 \\
\hline $\begin{array}{l}\text { Salix cinerea L. subsp. } \\
\text { oleifolia Macreight } \\
\text { (Salicaceae) BCN126656 }\end{array}$ & Gatell & $\mathrm{MO}$ & $\begin{array}{l}\text { Aerial part. } \\
\text { Stem }\end{array}$ & $\begin{array}{l}\text { Artisanal: basketry } \\
\text { Artisanal: cane elaboration } \\
\text { Ornamental: bouquet elaboration } \\
\text { Timber }\end{array}$ & 11 \\
\hline $\begin{array}{l}\text { Salix elaeagnos Scop. } \\
\text { subsp. angustifolia (Cariot) } \\
\text { Rech.f. (Salicaceae) } \\
\text { BCN31304 }\end{array}$ & $\begin{array}{l}\text { Salits, salitres, sàlix, sarga, } \\
\text { vergatera }\end{array}$ & $\mathrm{AE}, \mathrm{MO}$ & Stem & Artisanal: basketry & 7 \\
\hline $\begin{array}{l}\text { Salix ×fragilis L. (Salicaceae) } \\
\text { BCN31305 }\end{array}$ & $\begin{array}{l}\text { Saula, saule, salze dels vims, } \\
\text { saula vimera, vim, vimenera, } \\
\text { vímet }\end{array}$ & $\mathrm{AE}, \mathrm{MO}$ & Stem & $\begin{array}{l}\text { Artisanal: basketry } \\
\text { Artisanal: broom elaboration } \\
\text { Artisanal: footwear elaboration } \\
\text { Agrosilvopastoral management: for fishing } \\
\text { Ludic }\end{array}$ & 31 \\
\hline $\begin{array}{l}\text { Salsola kali L. subsp. } \\
\text { ruthenica (Iljin) Soó } \\
\text { (Amaranthaceae) } \\
\text { BCN42985 }\end{array}$ & Saleret, sosa & $\mathrm{Bl}$ & Not reported & $\begin{array}{l}\text { Cosmetic } \\
\text { Domestic: paint elaboration }\end{array}$ & 2 \\
\hline $\begin{array}{l}\text { Salsola soda } \mathrm{L} \text {. } \\
\text { (Amaranthaceae) } \\
\text { BCN42984 }\end{array}$ & Saleret & $\mathrm{Bl}$ & Not reported & Domestic: paint elaboration & 1 \\
\hline
\end{tabular}


Table 1 Non-food and non-medicinal folk plant uses in the territories of Alt Empordà, Montseny and Ripollès (Catalonia), and Formentera and Mallorca (Balearic Islands) (Continued)

\begin{tabular}{|c|c|c|c|c|c|}
\hline $\begin{array}{l}\text { Salsola vermiculata } \mathrm{L} \text {. } \\
\text { (Amaranthaceae) } \\
\text { BCN83712 }\end{array}$ & Barrilla, barrillera & $\mathrm{Bl}$ & Not reported & Other informations: ecological information & 1 \\
\hline $\begin{array}{l}\text { Salvia microphylla Kunth } \\
\text { (Lamiaceae) BCN126657 }\end{array}$ & $\begin{array}{l}\text { Gessamí, menta, menta de } \\
\text { la reina, menta piperita, } \\
\text { menta romana, menta } \\
\text { trapassera, ormí, senyorida }\end{array}$ & $\mathrm{BI}, \mathrm{MO}$ & $\begin{array}{l}\text { Flower. Whole } \\
\text { plant }\end{array}$ & $\begin{array}{l}\text { Agrosilvopastoral management: honey } \\
\text { obtaining } \\
\text { Ludic } \\
\text { Ornamental: gardening }\end{array}$ & 7 \\
\hline $\begin{array}{l}\text { Salvia officinalis L. } \\
\text { (Lamiaceae) BCN95591 }\end{array}$ & Salvi, sàlvia & $\mathrm{Bl}$ & $\begin{array}{l}\text { Aerial part. } \\
\text { Leaf. Not } \\
\text { reported. } \\
\text { Whole plant }\end{array}$ & $\begin{array}{l}\text { Agrosilvopastoral management: tools for } \\
\text { agricultural practices } \\
\text { Cosmetic } \\
\text { Domestic: for help at home } \\
\text { Folk oral literature } \\
\text { Ornamental: gardening } \\
\text { Other informations: ecological information } \\
\text { Other informations: appreciations on plant or } \\
\text { product properties }\end{array}$ & 17 \\
\hline $\begin{array}{l}\text { Salvia officinalis L. subsp. } \\
\text { lavandulifolia (Vahl) Gams } \\
\text { (Lamiaceae) BCN1674 }\end{array}$ & $\begin{array}{l}\text { Sàlvia, sàlvia femella, sàlvia } \\
\text { mascle }\end{array}$ & $\mathrm{MO}$ & Whole plant & Folk oral literature & 1 \\
\hline Salvia sp. (Lamiaceae) & Sàlvia & $\mathrm{Bl}$ & Whole plant & Other informations: ecological informations & 1 \\
\hline $\begin{array}{l}\text { Salvia verbenaca L. } \\
\text { (Lamiaceae) BCN29942 }\end{array}$ & Tàrrec, tàrrega & $\mathrm{Bl}, \mathrm{Rl}$ & $\begin{array}{l}\text { Leaf. Not } \\
\text { reported }\end{array}$ & Smoking & 5 \\
\hline $\begin{array}{l}\text { Sambucus nigra L. } \\
\text { (Adoxaceae) BCN29943 }\end{array}$ & $\begin{array}{l}\text { Sabuc, sabuquer, saüc, } \\
\text { saüquer }\end{array}$ & $A E, B I, R I$ & $\begin{array}{l}\text { Flower. Stem. } \\
\text { Whole plant }\end{array}$ & $\begin{array}{l}\text { Agrosilvopastoral management: tools for } \\
\text { agricultural practices } \\
\text { Domestic: air freshener } \\
\text { Ludic } \\
\text { Magic and religious beliefs and practices } \\
\text { Other informations: ecological information } \\
\text { Other informations: harvesting and/or selling }\end{array}$ & 9 \\
\hline $\begin{array}{l}\text { Santolina chamaecyparissus } \\
\text { L. (Asteraceae) BCN31526 }\end{array}$ & $\begin{array}{l}\text { Camamilla de botó, } \\
\text { camamilla de botó groc, } \\
\text { camamilla de l'hort, } \\
\text { espernallac }\end{array}$ & $\mathrm{MO}, \mathrm{RI}$ & $\begin{array}{l}\text { Aerial part. } \\
\text { Inflorescence }\end{array}$ & $\begin{array}{l}\text { Cosmetic } \\
\text { Domestic: air freshener } \\
\text { Folk oral literature } \\
\text { Ornamental: bouquet elaboration }\end{array}$ & 4 \\
\hline $\begin{array}{l}\text { Santolina chamaecyparissus } \\
\text { L. subsp. chamaecyparissus } \\
\text { (Asteraceae) BCN29782 }\end{array}$ & $\begin{array}{l}\text { Camamilla, camamilla de } \\
\text { muntanya }\end{array}$ & $\mathrm{AE}$ & $\begin{array}{l}\text { Aerial part. } \\
\text { Inflorescence }\end{array}$ & $\begin{array}{l}\text { Cosmetic } \\
\text { Ornamental: bouquet elaboration }\end{array}$ & 3 \\
\hline $\begin{array}{l}\text { Santolina chamaecyparissus } \\
\text { L. subsp. magonica O.Bolòs } \\
\text { \& al. (Asteraceae) } \\
\text { BCN-E-186 }\end{array}$ & Camamil la & $\mathrm{Bl}$ & $\begin{array}{l}\text { Aerial part. } \\
\text { Inflorescence. } \\
\text { Whole plant }\end{array}$ & $\begin{array}{l}\text { Agrosilvopastoral management: tools for } \\
\text { agricultural practices } \\
\text { Cosmetic } \\
\text { Domestic: air freshener } \\
\text { Folk oral literature } \\
\text { Magic and religious beliefs and practices } \\
\text { Other informations: ecological information } \\
\text { Repellent }\end{array}$ & 20 \\
\hline $\begin{array}{l}\text { Saponaria officinalis L. } \\
\text { (Caryophyllaceae) } \\
\text { BCN29783 }\end{array}$ & $\begin{array}{l}\text { Herba del sabó, herba } \\
\text { ensabonera, herba } \\
\text { sabonera, sabó, sabó de } \\
\text { gitana }\end{array}$ & $A E, M O, R l$ & $\begin{array}{l}\text { Aerial part. } \\
\text { Flower }\end{array}$ & $\begin{array}{l}\text { Cosmetic } \\
\text { Domestic: for help at home }\end{array}$ & 11 \\
\hline $\begin{array}{l}\text { Sarothamnus arboreus } \\
\text { (Desf.) Webb subsp. } \\
\text { catalaunicus (Webb) C.Vic } \\
\text { (Fabaceae) BCN29784 }\end{array}$ & $\begin{array}{l}\text { Ginesta borda, ginestell, } \\
\text { ginestó }\end{array}$ & $\mathrm{AE}$ & $\begin{array}{l}\text { Aerial part. } \\
\text { Stem. Whole } \\
\text { plant }\end{array}$ & $\begin{array}{l}\text { Agrosilvopastoral management: tools for } \\
\text { agricultural practices } \\
\text { Ornamental: bouquet elaboration } \\
\text { Other informations: ecological information }\end{array}$ & 3 \\
\hline $\begin{array}{l}\text { Sarothamnus scoparius (L.) } \\
\text { Wimm. ex Koch (Fabaceae) } \\
\text { BCN24988 }\end{array}$ & $\begin{array}{l}\text { Ginesta, ginesta prima, } \\
\text { ginestell, gódua, lliroia }\end{array}$ & $\mathrm{MO}, \mathrm{RI}$ & $\begin{array}{l}\text { Aerial part. } \\
\text { Flower }\end{array}$ & $\begin{array}{l}\text { Artisanal: broom elaboration } \\
\text { Domestic: for help at home } \\
\text { Fuel obtaining: charcoal } \\
\text { Magic and religious beliefs and practices } \\
\text { Ornamental: bouquet elaboration }\end{array}$ & 22 \\
\hline $\begin{array}{l}\text { Satureja calamintha (L.) } \\
\text { Scheele (Lamiaceae) } \\
\text { BCN109263 }\end{array}$ & Menta borda, rementerola & $\mathrm{MO}$ & Flower. Leaf & $\begin{array}{l}\text { Agrosilvopastoral management: agricultural/ } \\
\text { horticultural } \\
\text { Domestic: for help at home }\end{array}$ & 2 \\
\hline
\end{tabular}


Table 1 Non-food and non-medicinal folk plant uses in the territories of Alt Empordà, Montseny and Ripollès (Catalonia), and Formentera and Mallorca (Balearic Islands) (Continued)

\begin{tabular}{|c|c|c|c|c|c|}
\hline $\begin{array}{l}\text { Satureja calamintha (L.) } \\
\text { Scheele subsp. glandulosa } \\
\text { (Req.) Gams (Lamiaceae) } \\
\text { BCN29785 }\end{array}$ & Clements & $\mathrm{AE}$ & Stem & Domestic: for help at home & 1 \\
\hline $\begin{array}{l}\text { Satureja hortensis L. } \\
\text { (Lamiaceae) BCN29945 }\end{array}$ & Senyorida & $\mathrm{Bl}$ & Whole plant & Other informations: use not remembered & 1 \\
\hline $\begin{array}{l}\text { Saxifraga longifolia Lapeyr. } \\
\text { (Saxifragaceae) BCN54656 }\end{array}$ & Corona de rei & $\mathrm{Rl}$ & Whole plant & Ornamental: bouquet elaboration & 1 \\
\hline $\begin{array}{l}\text { Saxifraga vayredana Luizet } \\
\text { (Saxifragaceae) BCN95599 }\end{array}$ & Herba de Sant Segimon & $\mathrm{MO}$ & $\begin{array}{l}\text { Aerial part. } \\
\text { Whole plant }\end{array}$ & $\begin{array}{l}\text { Folk oral literature } \\
\text { Other informations: ecological information } \\
\text { Smoking }\end{array}$ & 11 \\
\hline $\begin{array}{l}\text { Scabiosa atropurpurea L. } \\
\text { (Dipsacaceae) BCN29947 }\end{array}$ & $\begin{array}{l}\text { Escabiosa, escabriosa, } \\
\text { escapiosa, herba escapriosa }\end{array}$ & $\mathrm{AE}, \mathrm{BI}$ & $\begin{array}{l}\text { Not reported. } \\
\text { Whole plant }\end{array}$ & Other informations: ecological information & 3 \\
\hline $\begin{array}{l}\text { Schefflera arborea (L.) } \\
\text { M.Gómez (Araliaceae) } \\
\text { BCN129084 }\end{array}$ & Xeflera & $\mathrm{Bl}$ & Whole plant & Ornamental: gardening & 1 \\
\hline $\begin{array}{l}\text { Schinus molle L. } \\
\text { (Anacardiaceae) BCN46086 }\end{array}$ & Pebre verd & $\mathrm{AE}$ & Aerial part & Ornamental: bouquet elaboration & 1 \\
\hline $\begin{array}{l}\text { Scirpus holoschoenus L. } \\
\text { (Cyperaceae) BCN29789 }\end{array}$ & Jonc & $\mathrm{AE}, \mathrm{Bl}, \mathrm{MO}$ & $\begin{array}{l}\text { Aerial part. } \\
\text { Stem }\end{array}$ & $\begin{array}{l}\text { Agrosilvopastoral management: tools for } \\
\text { agricultural practices } \\
\text { Agrosilvopastoral management: for fishing } \\
\text { Agrosilvopastoral management: for hunting } \\
\text { Agrosilvopastoral management: for pig } \\
\text { slaughter } \\
\text { Artisanal: basketry } \\
\text { Domestic: for help at home } \\
\text { Other informations: ecological information } \\
\text { Textile: fibre or cloth elaboration }\end{array}$ & 42 \\
\hline $\begin{array}{l}\text { Scolymus hispanicus L. } \\
\text { (Asteraceae) BCN30008 }\end{array}$ & $\begin{array}{l}\text { Card cadalina, card } \\
\text { catalina, card de } \\
\text { carxofa, card de } \\
\text { cadernina, card } \\
\text { cadernina, card verd }\end{array}$ & $\mathrm{Bl}$ & Whole plant & $\begin{array}{l}\text { Agrosilvopastoral management: for pig } \\
\text { slaughter } \\
\text { Folk oral literature } \\
\text { Other informations: ecological information } \\
\text { Other informations: use not remembered }\end{array}$ & 4 \\
\hline $\begin{array}{l}\text { Scrophularia nodosa L. } \\
\text { (Scrophulariaceae) } \\
\text { BCN3762 }\end{array}$ & Setge & $\mathrm{Rl}$ & Whole plant & Folk oral literature & 1 \\
\hline $\begin{array}{l}\text { Secale cereale L. (Poaceae) } \\
\text { BCN27243 }\end{array}$ & Secle, sègol & $\mathrm{MO}, \mathrm{Rl}$ & $\begin{array}{l}\text { Fruit. Stem. } \\
\text { Whole plant }\end{array}$ & $\begin{array}{l}\text { Domestic: for help at home } \\
\text { Ludic } \\
\text { Magic and religious beliefs and practices }\end{array}$ & 3 \\
\hline $\begin{array}{l}\text { Sechium edule (Jacq.) Sw. } \\
\text { (Cucurbitaceae) BC114504 }\end{array}$ & Tayote (Spanish) & $\mathrm{Bl}$ & Whole plant & Other informations: harvesting and/or selling & 1 \\
\hline $\begin{array}{l}\text { Sedum dasyphyllum L. } \\
\text { (Crassulaceae) BCN24994 }\end{array}$ & Arròs de paret & Rl & $\begin{array}{l}\text { Aerial part. } \\
\text { Leaf. Whole } \\
\text { plant }\end{array}$ & $\begin{array}{l}\text { Ludic } \\
\text { Ornamental: bouquet elaboration }\end{array}$ & 3 \\
\hline $\begin{array}{l}\text { Sedum rupestre L. } \\
\text { (Crassulaceae) BCN29791 }\end{array}$ & Mort-i-viu & $\mathrm{AE}$ & Aerial part & Magic and religious beliefs and practices & 1 \\
\hline $\begin{array}{l}\text { Sedum sediforme (Jacq.) } \\
\text { Pau (Crassulaceae) } \\
\text { BCN29792 }\end{array}$ & $\begin{array}{l}\text { Arròs, crespinell, mort-i-viu, } \\
\text { pinet, raïm de galàpet, raïm } \\
\text { de llop }\end{array}$ & $\mathrm{AE}, \mathrm{MO}$ & Aerial part & $\begin{array}{l}\text { Ludic } \\
\text { Magic and religious beliefs and practices } \\
\text { Ornamental: bouquet elaboration }\end{array}$ & 4 \\
\hline $\begin{array}{l}\text { Sedum telephium L. } \\
\text { (Crassulaceae) BCN24995 }\end{array}$ & Matafoc & $\mathrm{Rl}$ & Whole plant & Ornamental: bouquet elaborations & 1 \\
\hline $\begin{array}{l}\text { Sedum telephium L. subsp. } \\
\text { maximum (Crassulaceae) } \\
\text { BCN32123 }\end{array}$ & $\begin{array}{l}\text { Bàlsam, bàlsam de fulla, } \\
\text { bàlsam de jardí, fava de } \\
\text { jardí, fava grassa }\end{array}$ & $\mathrm{MO}$ & Whole plant & Ornamental: gardening & 2 \\
\hline \multirow{2}{*}{$\begin{array}{l}\text { Sempervirum } \\
\text { arachnoideum L. } \\
\text { (Crassulaceae) BCN26422 }\end{array}$} & Vel de núvia & $\mathrm{Rl}$ & Whole plant & Ornamental: gardening & 1 \\
\hline & & $\mathrm{MO}, \mathrm{Rl}$ & Whole plant & Ornamental: bouquet elaboration & 4 \\
\hline
\end{tabular}


Table 1 Non-food and non-medicinal folk plant uses in the territories of Alt Empordà, Montseny and Ripollès (Catalonia), and Formentera and Mallorca (Balearic Islands) (Continued)

\section{Sempervirum tectorum $\mathrm{L}$. (Crassulaceae) BCN24997 \\ Senecio inaequidens DC. (Asteraceae) BCN31309 \\ Sequoiadenderon \\ giganteum (Lindl.) \\ J.Buchholz (Taxodiaceae) BCN27276 \\ Setaria viridis (L.) Beauv. (Poaceae) BCN52592}

Sideritis cf. sventenii (Kunkel) Mend.-Heu (Lamiaceae) BCN29957

Silene vulgaris (Moench) Garcke (Caryophyllaceae) BCN29948

Silene vulgaris (Moench) Garcke subsp. vulgaris

(Caryophyllaceae) BCN25001

Sinapis alba L. (Brassicaceae) BCN50358

Smilax aspera L. (Smilacaceae) BCN2115

Smyrnium olusatrum L. (Apiaceae) BNC95551

Solanum linnaeanum Hepper \& P.-M.L.Jaeger (Solanaceae) BCN2553

Solanum lycopersicum L. (Solanaceae) BCN29952

Solanum melongena $\mathrm{L}$. (Solanaceae) BCN25004

Solanum tuberosum L. (Solanaceae) BCN29797

Sonchus oleraceus (L.) L. (Asteraceae) BCN29953

Sonchus tenerrimus $\mathrm{L}$. (Asteraceae) BCN29954

Sorbus aucuparia L. (Rosaceae) BCN25009
Apagafoc, consolva, herba

de les cremades, matafoc,

pinya de foc, sempervívum

Seneci del Cap

$A E$

Whole plant

Other informations: ecological information

Sequoia

RI

Bark

Ludic

Cua de mula, panissola, pèl de boc, xerreix, xerreix bord

$\begin{array}{lll}- & \text { AE } & \text { Aerial part } \\ \begin{array}{l}\text { Colís, colitx, colleja } \\ \text { (Spanish), culivells, } \\ \text { esclafidor, petapetó } \\ \text { Corretjola, esclafidor, pet }\end{array} & \text { RI } & \begin{array}{l}\text { Aerial part. } \\ \text { Flower }\end{array} \\ & & \begin{array}{l}\text { Aerial part. } \\ \text { Flower. Leaf. } \\ \text { Whole plant }\end{array} \\ \text { Mostassa } & \text { Bl } & \text { Whole plant } \\ \text { Arijol, aritja, sarsaparrilla } & \text { AE, Bl } & \begin{array}{l}\text { Aerial part. } \\ \text { Root. Whole } \\ \text { plant }\end{array}\end{array}$

Aleixandre, aleixandri

BI

Metzinera, poma del desert

BI

Stem

Whole plant

Domàtiga (fruit),

$\mathrm{AE}, \mathrm{BI} \quad$ Fruit. Leaf. Whole plant

omatiguera, tomata (fruit), tomatera, tomàtiga (fruit), tomatiguera,

\section{Albergínia (fruit)} alberginiera

Fruit. Leaf. Whole plant

Patata (tuber), patatera
$A E, B I, M O \quad$ Leaf. Stem. Tuber. Whole plant

Ludic

Ornamental: bouquet elaboration

Other informations: use not remembered

Ludic

Agrosilvopastoral management: tools for agricultural practices

Repellent

Agrosilvopastoral management: for pig

slaughter

Domestic: for help at home

Ornamental: bouquet elaboration

Other informations: ecological information

Other informations: use not remembered

Textile: textile padding

Artisanal: broom elaboration

Ornamental: bouquet elaboration

Other informations: use not remembered

Agrosilvopastoral management: agricultural/ horticultural

Domestic: for help at home

Other informations: ecological information

Other informations: harvesting and/or selling

Repellent

Smoking

Agrosilvopastoral management: agricultural/ horticultural

Magic and religious beliefs and practices Smoking

Agrosilvopastoral management: agricultural/ 7 horticultural

Domestic: for help at home

Magic and religious beliefs and practices Smoking

Allitsó, allitsó vertader,

$\mathrm{BI}$

Latex. Whole

Other informations: ecological information alitxó, aritsó, lletsó, lletxó, llitsó, llitxó

plan

Aletxó, llitsó bord

Leaf

Other informations: ecological information 
Table 1 Non-food and non-medicinal folk plant uses in the territories of Alt Empordà, Montseny and Ripollès (Catalonia), and Formentera and Mallorca (Balearic Islands) (Continued)

\begin{tabular}{|c|c|c|c|c|c|}
\hline $\begin{array}{l}\text { Sorbus domestica L. } \\
\text { (Rosaceae) BCN46827 }\end{array}$ & Serva (fruit), server, servera & $\mathrm{AE}, \mathrm{Bl}, \mathrm{MO}$ & $\begin{array}{l}\text { Stem. Whole } \\
\text { plant }\end{array}$ & $\begin{array}{l}\text { Agrosilvopastoral management: tools for } \\
\text { agricultural practices } \\
\text { Other informations: ecological information } \\
\text { Timber }\end{array}$ & 7 \\
\hline $\begin{array}{l}\text { Sorghum bicolor }(\mathrm{L} .) \\
\text { Moench (Poaceae) } \\
\text { BCN31310 }\end{array}$ & Melca & $\mathrm{AE}$ & Stem & Artisanal: broom elaboration & 13 \\
\hline $\begin{array}{l}\text { Sorghum halepense }(\mathrm{L} .) \\
\text { Pers. (Poaceae) BCN44695 }\end{array}$ & Canyota & $\mathrm{MO}$ & Aerial part & Ornamental: bouquet elaboration & 2 \\
\hline $\begin{array}{l}\text { Spartium junceum L. } \\
\text { (Fabaceae) BCN29956 }\end{array}$ & $\begin{array}{l}\text { Ginesta, ginesta de llei, } \\
\text { ginesta vera, ginestera }\end{array}$ & $\begin{array}{l}\mathrm{AE}, \mathrm{BI} \\
\mathrm{MO}, \mathrm{RI}\end{array}$ & $\begin{array}{l}\text { Aerial part. } \\
\text { Flower. Stem. } \\
\text { Whole plant }\end{array}$ & $\begin{array}{l}\text { Agrosilvopastoral management: tools for } \\
\text { agricultural practices } \\
\text { Artisanal: broom elaboration } \\
\text { Artisanal: musical instrument elaboration } \\
\text { Domestic: for help at home } \\
\text { Magic and religious beliefs and practices } \\
\text { Ornamental: bouquet elaboration } \\
\text { Ornamental: gardening } \\
\text { Other informations }\end{array}$ & 27 \\
\hline $\begin{array}{l}\text { Spinacia oleracea L. } \\
\text { (Amaranthaceae) } \\
\text { BCN46077 }\end{array}$ & Espinac & $\mathrm{Bl}$ & Leaf & $\begin{array}{l}\text { Other informations: appreciations on plant or } \\
\text { product properties }\end{array}$ & 1 \\
\hline $\begin{array}{l}\text { Stachys byzantina K.Koch } \\
\text { (Lamiaceae) BCN126667 }\end{array}$ & $\begin{array}{l}\text { Bàlsam, bàlsam de tall, } \\
\text { bàlsam pelut, herba peluda, } \\
\text { orella de conill }\end{array}$ & $\mathrm{MO}$ & Whole plant & Ornamental: gardening & 2 \\
\hline $\begin{array}{l}\text { Stipa tenacissima L. } \\
\text { (Poaceae) BCN46091 }\end{array}$ & Clin, espart & $\mathrm{AE}, \mathrm{BI}, \mathrm{MO}$ & $\begin{array}{l}\text { Aerial part. } \\
\text { Stem. Whole } \\
\text { plant }\end{array}$ & $\begin{array}{l}\text { Agrosilvopastoral management: tools for } \\
\text { agricultural practices } \\
\text { Agrosilvopastoral management: for hunting } \\
\text { Artisanal: basketry } \\
\text { Artisanal: footwear elaboration } \\
\text { Domestic: for help at home } \\
\text { Magic and religious beliefs and practices } \\
\text { Other informations: use not remembered } \\
\text { Textile: textile padding } \\
\text { Timber: boat manufacturing }\end{array}$ & 11 \\
\hline $\begin{array}{l}\text { Syringa vulgaris L. } \\
\text { (Oleaceae) BCN29959 }\end{array}$ & Lilà & $\mathrm{AE}, \mathrm{MO}, \mathrm{RI}$ & $\begin{array}{l}\text { Aerial part. } \\
\text { Flower. Whole } \\
\text { plant }\end{array}$ & $\begin{array}{l}\text { Folk oral literature } \\
\text { Magic and religious beliefs and practices } \\
\text { Ornamental: bouquet elaboration } \\
\text { Ornamental: gardening }\end{array}$ & 17 \\
\hline $\begin{array}{l}\text { Syzygium aromaticum (L.) } \\
\text { Merr. \& L.M.Perry } \\
\text { (Myrtaceae) BCN47279 }\end{array}$ & Clau, clau d'olor & $\mathrm{Bl}$ & Floral bud & $\begin{array}{l}\text { Domestic: air freshener } \\
\text { Repellent }\end{array}$ & 2 \\
\hline $\begin{array}{l}\text { Tamarix canariensis Willd. } \\
\text { (Tamariaceae) BCN43378 }\end{array}$ & Tamariu & $\mathrm{AE}$ & Whole plant & Folk oral literature & 1 \\
\hline $\begin{array}{l}\text { Tanacetum corymbosum } \\
\text { (L.) Sch.Bip. (Asteraceae) } \\
\text { BCN31918 }\end{array}$ & - & $\mathrm{MO}$ & Whole plant & Other informations: use not remembered & 1 \\
\hline $\begin{array}{l}\text { Tanacetum parthenium (L.) } \\
\text { Sch.Bip. (Asteraceae) } \\
\text { BCN25014 }\end{array}$ & $\begin{array}{l}\text { Camamilla amargant, } \\
\text { camamiglla borda, } \\
\text { camamilla de Sòria, } \\
\text { camamilla purgant, } \\
\text { camamil · la de jardí, } \\
\text { botonets }\end{array}$ & $\mathrm{Bl}, \mathrm{MO}, \mathrm{Rl}$ & $\begin{array}{l}\text { Aerial part. } \\
\text { Whole plant }\end{array}$ & $\begin{array}{l}\text { Ornamental: bouquet elaboration } \\
\text { Ornamental: gardening }\end{array}$ & 3 \\
\hline $\begin{array}{l}\text { Tanacetum vulgare L. } \\
\text { (Asteraceae) BCN29803 }\end{array}$ & $\begin{array}{l}\text { Danarida, tanaceto } \\
\text { (Spanish), tanarida }\end{array}$ & $\mathrm{Bl}, \mathrm{RI}$ & $\begin{array}{l}\text { Aerial part. } \\
\text { Whole plant }\end{array}$ & $\begin{array}{l}\text { Agrosilvopastoral management: agricultural/ } \\
\text { horticultural } \\
\text { Ornamental: bouquet elaboration } \\
\text { Repellent }\end{array}$ & 4 \\
\hline
\end{tabular}


Table 1 Non-food and non-medicinal folk plant uses in the territories of Alt Empordà, Montseny and Ripollès (Catalonia), and Formentera and Mallorca (Balearic Islands) (Continued)

\begin{tabular}{|c|c|c|c|c|c|}
\hline $\begin{array}{l}\text { Taraxacum officinale Weber } \\
\text { in Wiggers (Asteraceae) } \\
\text { BCN25015 }\end{array}$ & $\begin{array}{l}\text { Angelets, dent de lleó, } \\
\text { herba queixalera, lleones, } \\
\text { llums, màstecs bords, } \\
\text { paraigües, pixacà, } \\
\text { pixaconills, pixallits, puputs, } \\
\text { queixal de llop, xicoina }\end{array}$ & $\mathrm{Bl}, \mathrm{MO}, \mathrm{Rl}$ & $\begin{array}{l}\text { Aerial part. } \\
\text { Inflorescence. } \\
\text { Fruit. Whole } \\
\text { plant }\end{array}$ & $\begin{array}{l}\text { Cosmetic } \\
\text { Ludic } \\
\text { Magic and religious beliefs and practices }\end{array}$ & 8 \\
\hline $\begin{array}{l}\text { Taxus baccata L. (Taxaceae) } \\
\text { BCN25017 }\end{array}$ & Teix, quiner & $\mathrm{Bl}, \mathrm{MO}, \mathrm{Rl}$ & $\begin{array}{l}\text { Aril. Cortical } \\
\text { parenchyma. } \\
\text { Stem. Whole } \\
\text { plant }\end{array}$ & $\begin{array}{l}\text { Cosmetic } \\
\text { Folk oral literature } \\
\text { Ludic } \\
\text { Magic and religious beliefs and practices } \\
\text { Other informations: ecological information } \\
\text { Timber: furniture manufacturing }\end{array}$ & 6 \\
\hline $\begin{array}{l}\text { Teucrium capitatum L. } \\
\text { (Lamiaceae) BCN43441 }\end{array}$ & $\begin{array}{l}\text { Herba de Sant Ponç, } \\
\text { mançanella }\end{array}$ & $\mathrm{Bl}$ & Aerial part & $\begin{array}{l}\text { Ornamental: bouquet elaboration } \\
\text { Other informations: use not remembered }\end{array}$ & 2 \\
\hline $\begin{array}{l}\text { Teucrium chamaedrys L. } \\
\text { (Lamiaceae) BC806359 }\end{array}$ & Auladella, betera & $\mathrm{Bl}$ & Whole plant & Other informations & 1 \\
\hline $\begin{array}{l}\text { Teucrium chamaedrys L. } \\
\text { subsp. pinnatifidum } \\
\text { (Sennen) Rech.f. } \\
\text { (Lamiaceae) BCN126671 }\end{array}$ & Brutònica & $\mathrm{MO}$ & Flower & $\begin{array}{l}\text { Agrosilvopastoral management: tools for } \\
\text { agricultural practices } \\
\text { Other informations: ecological information }\end{array}$ & 1 \\
\hline $\begin{array}{l}\text { Teucrium marum L. subsp. } \\
\text { subspinosum (Pourr. ex } \\
\text { Willd.) O.Bolòs, Molin. \& } \\
\text { P.Monts. (Lamiaceae) } \\
\text { BCN66404 }\end{array}$ & $\begin{array}{l}\text { Auladella, beteta, coixinet } \\
\text { de monja, eixorba-rates, } \\
\text { herba beteta, herba de Sant } \\
\text { Ponç, poniol }\end{array}$ & $\mathrm{Bl}$ & Whole plant & $\begin{array}{l}\text { Agrosilvopastoral management: weeds } \\
\text { Folk oral literature }\end{array}$ & 2 \\
\hline $\begin{array}{l}\text { Teucrium polium L. subsp. } \\
\text { capitatum (L.) Arcang. } \\
\text { (Lamiaceae) BCN103557 }\end{array}$ & $\begin{array}{l}\text { Auladella, beteta, coixinet } \\
\text { de monja, eixorba-rates, } \\
\text { herba beteta, herba de Sant } \\
\text { Ponç, poniol }\end{array}$ & $\mathrm{Bl}$ & Aerial part & Ornamental: bouquet elaboration & 1 \\
\hline $\begin{array}{l}\text { Teucrium polium L. subsp. } \\
\text { polium (Lamiaceae) } \\
\text { BCN32116 }\end{array}$ & Timó, timolet, timonet & $\mathrm{MO}$ & Flower & $\begin{array}{l}\text { Agrosilvopastoral management: tools for } \\
\text { agricultural practices }\end{array}$ & 1 \\
\hline $\begin{array}{l}\text { Thapsia villosa L. subsp. } \\
\text { villosa (Apiaceae) } \\
\text { BCN39998 }\end{array}$ & Julivert de cavall & $\mathrm{AE}$ & Whole plant & Other informations: ecological information & 1 \\
\hline $\begin{array}{l}\text { Thymbra capitata (L.) Cav. } \\
\text { (Lamiaceae) BCN72716 }\end{array}$ & Frígola & $\mathrm{Bl}$ & Leaf & Domestic: for help at home & 1 \\
\hline $\begin{array}{l}\text { Thymus vulgaris L. } \\
\text { (Lamiaceae) BCN29961 }\end{array}$ & $\begin{array}{l}\text { Farigola, frigola, tem, } \\
\text { tomillo (Spanish) }\end{array}$ & $\mathrm{AE}, \mathrm{BI}, \mathrm{RI}$ & $\begin{array}{l}\text { Aerial part. } \\
\text { Flower }\end{array}$ & $\begin{array}{l}\text { Cosmetic } \\
\text { Domestic: air freshener } \\
\text { Magic and religious beliefs and practices } \\
\text { Other informations: excipient or adjuvant in } \\
\text { medicinal or food preparations }\end{array}$ & 8 \\
\hline $\begin{array}{l}\text { Thymus vulgaris L. subsp. } \\
\text { vulgaris (Lamiaceae) } \\
\text { BCN44665 }\end{array}$ & Farigola & $\mathrm{MO}$ & Aerial part & $\begin{array}{l}\text { Agrosilvopastoral management: agricultural/ } \\
\text { horticultural } \\
\text { Folk oral literature }\end{array}$ & 6 \\
\hline $\begin{array}{l}\text { Tilia cordata Mill. } \\
\text { (Malvaceae) BCN26784 }\end{array}$ & Til $\cdot 10$ & $\mathrm{Bl}$ & $\begin{array}{l}\text { Bract with } \\
\text { flower }\end{array}$ & Other informations: harvesting and/or selling & 2 \\
\hline $\begin{array}{l}\text { Tilia platyphyllos Scop. } \\
\text { (Malvaceae) BCN25024 }\end{array}$ & Flor de tei, tell, til · la & $A E, R I$ & $\begin{array}{l}\text { Inflorescence. } \\
\text { Whole plant }\end{array}$ & $\begin{array}{l}\text { Ornamental: gardening } \\
\text { Other informations: harvesting and/or selling }\end{array}$ & 3 \\
\hline Tilia sp. (Malvaceae) & Til $\cdot$ la & $\mathrm{Bl}$ & Whole plant & Other informations: use not remembered & 1 \\
\hline $\begin{array}{l}\text { Tillandsia sp. } \\
\text { (Bromeliaceae) }\end{array}$ & Clavell de l'aire & $\mathrm{Bl}$ & Whole plant & Ornamental: gardening & 1 \\
\hline $\begin{array}{l}\text { Tradescantia albiflora } \\
\text { Kunth. (Commelinaceae) } \\
\text { BCN49624 }\end{array}$ & - & $\mathrm{MO}$ & Whole plant & Ornamental: gardening & 1 \\
\hline $\begin{array}{l}\text { Tribulus terrestris L. } \\
\text { (Zygophyllaceae) } \\
\text { BCN45017 }\end{array}$ & Picatalons & $\mathrm{Bl}$ & Whole plant & Agrosilvopastoral management: weeds & 1 \\
\hline
\end{tabular}


Table 1 Non-food and non-medicinal folk plant uses in the territories of Alt Empordà, Montseny and Ripollès (Catalonia), and Formentera and Mallorca (Balearic Islands) (Continued)

\begin{tabular}{|c|c|c|c|c|c|}
\hline $\begin{array}{l}\text { Trichocereus pachanoi } \\
\text { Britton \& Rose (Cactaceae) } \\
\text { BCN129086 }\end{array}$ & $\begin{array}{l}\text { Dompedro (Spanish), } \\
\text { sampedro (Spanish) }\end{array}$ & $\mathrm{Bl}$ & $\begin{array}{l}\text { Aerial part. } \\
\text { Whole plant }\end{array}$ & $\begin{array}{l}\text { Magic and religious beliefs and practices } \\
\text { Ornamental: gardening } \\
\text { Other informations: appreciations on plant or } \\
\text { product properties }\end{array}$ & 4 \\
\hline Trifolium sp. (Fabaceae) & Trèvol & $\mathrm{Bl}$ & $\begin{array}{l}\text { Aerial part. } \\
\text { Whole plant }\end{array}$ & $\begin{array}{l}\text { Agrosilvopastoral management: tools for } \\
\text { agricultural practices } \\
\text { Fuel obtaining: charcoal } \\
\text { Other informations: ecological information }\end{array}$ & 4 \\
\hline $\begin{array}{l}\text { Triticum aestivum L. } \\
\text { (Poaceae) BCN29963 }\end{array}$ & Blat, segó & $\begin{array}{l}\mathrm{AE}, \mathrm{BI} \\
\mathrm{MO}, \mathrm{RI}\end{array}$ & $\begin{array}{l}\text { Aerial part. } \\
\text { Bran. Not } \\
\text { reported. Fruit. } \\
\text { Spike. Stem. } \\
\text { Whole plant }\end{array}$ & $\begin{array}{l}\text { Agrosilvopastoral management: agricultural/ } \\
\text { horticultural } \\
\text { Agrosilvopastoral management: for fishing } \\
\text { Artisanal: footwear elaboration } \\
\text { Cosmetic } \\
\text { Domestic: for help at home } \\
\text { Folk oral literature } \\
\text { Magic and religious beliefs and practices } \\
\text { Ornamental: bouquet elaboration } \\
\text { Other informations: ecological information } \\
\text { Other informations: collections and/or selling } \\
\text { Other informations: use not rememberd }\end{array}$ & 24 \\
\hline $\begin{array}{l}\text { Trollius europaeus L. } \\
\text { (Ranunculaceae) BCN27267 }\end{array}$ & $\begin{array}{l}\text { Cloclou, flor de Sant Pere, } \\
\text { rovell d'ou }\end{array}$ & $\mathrm{Rl}$ & Aerial part & Ornamental: bouquet elaboration & 2 \\
\hline $\begin{array}{l}\text { Tropaeolum majus L. } \\
\text { (Tropaeolaceae) BCN46429 }\end{array}$ & Canari, caputxina & $\mathrm{BI}, \mathrm{MO}$ & Whole plant & $\begin{array}{l}\text { Agrosilvopastoral management: tools for } \\
\text { agricultural practices } \\
\text { Ornamental: gardening }\end{array}$ & 2 \\
\hline $\begin{array}{l}\text { Tulbaghia sp. } \\
\text { (Amaryllidaceae) }\end{array}$ & Tulbàgia & $\mathrm{Bl}$ & Whole plant & $\begin{array}{l}\text { Agrosilvopastoral management: tools for } \\
\text { agricultural practices } \\
\text { Repellent }\end{array}$ & 2 \\
\hline Tulipa sp. (Liliaceae) & Tulipán (Spanish) & $\mathrm{Bl}$ & Whole plant & Ornamental: gardening & 1 \\
\hline $\begin{array}{l}\text { Typha angustifolia L. } \\
\text { (Typhaceae) BCN23969 }\end{array}$ & Boga, bova & $\mathrm{BI}, \mathrm{MO}$ & $\begin{array}{l}\text { Aerial part. } \\
\text { Leaf. Stem }\end{array}$ & $\begin{array}{l}\text { Folk oral literature } \\
\text { Ornamental: bouquet elaboration } \\
\text { Textile: fibre or cloth elaboration }\end{array}$ & 12 \\
\hline $\begin{array}{l}\text { Typha angustifolia L. subsp. } \\
\text { australis (Schumach.) Kronf. } \\
\text { (Typhaceae) BCN51627 }\end{array}$ & Bova & $\mathrm{Bl}$ & Stem & $\begin{array}{l}\text { Artisanal: basketry } \\
\text { Other informations: ecological information } \\
\text { Textile }\end{array}$ & 3 \\
\hline $\begin{array}{l}\text { Typha latifolia L. } \\
\text { (Typhaceae) BCN31314 }\end{array}$ & Balca, boga, bova & $\mathrm{AE}, \mathrm{Bl}, \mathrm{MO}$ & $\begin{array}{l}\text { Aerial part. } \\
\text { Flower. Leaf. } \\
\text { Stem }\end{array}$ & $\begin{array}{l}\text { Agrosilvopastoral management: tools for } \\
\text { agricultural practices } \\
\text { Artisanal: basketry } \\
\text { Folk oral literature } \\
\text { Ludic } \\
\text { Ornamental: bouquet elaboration } \\
\text { Other informations: ecological information } \\
\text { Textile: fibre or cloth elaboration } \\
\text { Timber: furniture manufacturing }\end{array}$ & 23 \\
\hline $\begin{array}{l}\text { Ulex parviflorus Pourr. } \\
\text { subsp. parviflorus } \\
\text { (Fabaceae) BCN30011 }\end{array}$ & Argelac, argentina & $\mathrm{AE}$ & $\begin{array}{l}\text { Stem. Whole } \\
\text { plant }\end{array}$ & $\begin{array}{l}\text { Fuel obtaining: firewood } \\
\text { Other informations: ecological information }\end{array}$ & 2 \\
\hline $\begin{array}{l}\text { Ulmus minor Mill. } \\
\text { (Ulmaceae) BCN29813 }\end{array}$ & Om & $\begin{array}{l}\mathrm{AE}, \mathrm{BI} \\
\mathrm{MO}, \mathrm{RI}\end{array}$ & Bark. Stem & $\begin{array}{l}\text { Agrosilvopastoral management: agricultural/ } \\
\text { horticultural } \\
\text { Artisanal: cane elaboration } \\
\text { Artisanal: cookware elaboration } \\
\text { Folk oral literature } \\
\text { Textile: fibre or cloth elaboration } \\
\text { Timber } \\
\text { Timber: boat manufacturing } \\
\text { Timber: wheeled vehicles manufacturing } \\
\text { Timber: furniture manufacturing } \\
\text { Timber: construction materials production }\end{array}$ & 20 \\
\hline
\end{tabular}


Table 1 Non-food and non-medicinal folk plant uses in the territories of Alt Empordà, Montseny and Ripollès (Catalonia), and Formentera and Mallorca (Balearic Islands) (Continued)

Urginea maritima (L.) Baker Ceba marina, ceba marinera BI

(Asparagaceae) BCN58049

$\begin{array}{ll}\text { Urtica dioica L. (Urticaceae) } & \text { Ortiga } \\ \text { BCN25030 } & \\ \text { Urtica membranacea Poir. } & \text { Ortiga } \\ \text { ex Savigny (Urticaceae) } & \\ \text { BCN95578 } & \\ \text { Urtica sp. (Urticaceae) } & \text { Ortiga } \\ & \\ \text { Urtica urens L. (Urticaceae) } & \text { Estrígol, ortiga } \\ \text { BCN29966 } & \end{array}$

Valeriana officinalis L. (Caprifoliaceae) BCN29816

Verbascum pulverulentum Vill. (Scrophulariaceae) BCN25035

Verbascum sinuatum L. (Scrophulariaceae) BCN29967

Verbascum sp. (Scrophulariaceae)

Verbascum thapsus L. (Scrophulariaceae) BCN63299

Verbascum thapsus $\mathrm{L}$. subsp. montanum (Schard.) Bon (Scrophulariaceae) BCN39651

Viburnum lantana L. (Adoxaceae) BCN27281

Viburnum opulus L. (Adoxaceae) BCN126683

Viburnum opulus L. var. roseum Aiton (Adoxaceae) BCN27254

Viburnum tinus L. subsp. tinus (Adoxaceae) BCN126681

Vicia cracca L. subsp. gerardii (W.D.J. Koch) Briq. (Fabaceae) BCN126685

Vicia faba L. (Fabaceae) BCN49339

\section{Valedriana, valeriana}

Herba ploranera, torpa

Trepó, tripó

Croca, cua de guilla, trepó

Porpra, trepó

Candeler, trepó

BI

$\mathrm{AE}, \mathrm{BI} \quad$ Aerial part

Leaf. Not reported. Stem. Whole plant

AE, MO, RI Root

RI Aerial part

$A E, B I$

Root. Stem. Whole plant

$\mathrm{Bl}, \mathrm{Rl}$,

Aerial part Leaf. Not reported. Whole plant

Flower

Flower. Leaf

Cartellatge, tortellatge

Bola de neu, mató, pompa

$\mathrm{MO}, \mathrm{RI}$

Aerial part. Whole plant

Mató

RI

Aerial part

$\mathrm{MO}$

Llegum, veça

Fava (fruit/seed), favera, favó (seed), llegum

Aerial part. Whole plant

MO Aerial part

Bl, RI Fruit. Seed.
Agrosilvopastoral management: tools for

agricultural practices

Ornamental: gardening

Other informations: ecological information Repellent

Folk oral literature

Magic and religious beliefs and practices

Agrosilvopastoral management: tools for agricultural practices

Cosmetic

Other informations

Agrosilvopastoral management: tools for agricultural practices

Folk oral literature

Magic and religious beliefs and practices Other informations: undesirable actions Repellent

Agrosilvopastoral management: tools for agricultural practices

Cosmetic

Other informations: undesirable actions Repellent

Folk oral literature

Agrosilvopastoral management: weeds Magic and religious beliefs and practices

Artisanal: broom elaboration

Folk oral literature

Magic and religious beliefs and practices

Domestic: for help at home

Magic and religious beliefs and practices Other informations: ecological information

Cosmetic

Folk oral literature

Other informations: ecological information

Agrosilvopastoral management: tools for

Artisanal: cane elaboration

Magic and religious beliefs and practices

Ornamental: bouquet elaboration

Ornamental: gardening

Magic and religious beliefs and practices

Agrosilvopastoral management: tools for agricultural practices Ornamental: gardening

Magic and religious beliefs and practices

1

Agrosilvopastoral management: agricultural/ horticultural

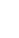

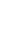

(n)


Table 1 Non-food and non-medicinal folk plant uses in the territories of Alt Empordà, Montseny and Ripollès (Catalonia), and Formentera and Mallorca (Balearic Islands) (Continued)

\begin{tabular}{|c|c|c|c|c|c|}
\hline & & & & $\begin{array}{l}\text { Agrosilvopastoral management: honey } \\
\text { obtaining } \\
\text { Folk oral literature } \\
\text { Magic and religious beliefs and practices } \\
\text { Other informations: harvesting and/or selling }\end{array}$ & \\
\hline $\begin{array}{l}\text { Vicia sativa L. (Fabaceae) } \\
\text { BCN95569 }\end{array}$ & Veça & $\mathrm{Bl}$ & Young shoot & $\begin{array}{l}\text { Magic and religious beliefs and practices } \\
\text { Ornamental: bouquet elaboration }\end{array}$ & 2 \\
\hline $\begin{array}{l}\text { Vicia sativa L. subsp. sativa } \\
\text { (Fabaceae) BCN-E-93 }\end{array}$ & Veça & MO & Whole plant & Ornamental: gardening & 1 \\
\hline $\begin{array}{l}\text { Vicia villosa Roth } \\
\text { (Fabaceae) BCF46443 }\end{array}$ & Veça & $\mathrm{MO}$ & Whole plant & Magic and religious beliefs and practices & 1 \\
\hline $\begin{array}{l}\text { Vinca difformis Pourr. } \\
\text { (Apocynaceae) BCN52597 }\end{array}$ & $\begin{array}{l}\text { Blincaperblinca, proenga, } \\
\text { pruenga, vinca, } \\
\text { vincapervinca }\end{array}$ & $\mathrm{Bl}, \mathrm{MO}, \mathrm{Rl}$ & $\begin{array}{l}\text { Aerial part. } \\
\text { Whole plant }\end{array}$ & $\begin{array}{l}\text { Folk oral literature } \\
\text { Ornamental: bouquet elaboration } \\
\text { Other informations: ecological information }\end{array}$ & 5 \\
\hline $\begin{array}{l}\text { Vinca major L. } \\
\text { (Apocynaceae) BCN25039 }\end{array}$ & $\begin{array}{l}\text { Flor de Pasqua, vinca, } \\
\text { vincapervinca }\end{array}$ & $\mathrm{AE}, \mathrm{Rl}$ & $\begin{array}{l}\text { Aerial part. } \\
\text { Whole plant }\end{array}$ & $\begin{array}{l}\text { Ornamental: bouquet elaboration } \\
\text { Ornamental: gardening }\end{array}$ & 4 \\
\hline $\begin{array}{l}\text { Viola alba Besser } \\
\text { (Violaceae) BCN27286 }\end{array}$ & Violetes & $\mathrm{Rl}$ & Flower & Ornamental: bouquet elaboration & 1 \\
\hline $\begin{array}{l}\text { Viola alba Besser. subsp. } \\
\text { dehnhardtii (Ten.) W.Becker } \\
\text { (Violaceae) BCN31316 }\end{array}$ & Viola, viola petita & $\mathrm{AE}$ & Aerial part & Ornamental: bouquet elaboration & 1 \\
\hline $\begin{array}{l}\text { Viscum album L. } \\
\text { (Santalaceae) BCN46085 }\end{array}$ & $\begin{array}{l}\text { Gui (French), muérdago } \\
\text { (Spanish), ramillo de la } \\
\text { suerte (Spanish), vesc }\end{array}$ & $A E, M O$ & $\begin{array}{l}\text { Aerial part. } \\
\text { Resin. Whole } \\
\text { plant }\end{array}$ & $\begin{array}{l}\text { Agrosilvopastoral management: for hunting } \\
\text { Magic and religious beliefs and practices } \\
\text { Ornamental: bouquet elaboration }\end{array}$ & 7 \\
\hline $\begin{array}{l}\text { Vitex agnus-castus L. } \\
\text { (Lamiaceae) BCN29820 }\end{array}$ & $\begin{array}{l}\text { Alís d'olor, aloc, barda, } \\
\text { ximbla }\end{array}$ & $\mathrm{AE}, \mathrm{Bl}$ & $\begin{array}{l}\text { Flower. Latex. } \\
\text { Stem }\end{array}$ & $\begin{array}{l}\text { Agrosilvopastoral management: tools for } \\
\text { agricultural practices } \\
\text { Ludic } \\
\text { Magic and religious beliefs and practices } \\
\text { Other informations: use not remembered } \\
\text { Repellent }\end{array}$ & 5 \\
\hline $\begin{array}{l}\text { Vitis vinifera L. (Vitacaeae) } \\
\text { BCN29972 }\end{array}$ & $\begin{array}{l}\text { Cep, pansa (dried fruit), } \\
\text { tòria (branch), parra, raïm } \\
\text { (fruit), raïmera, rem (fruit), vi } \\
\text { (elaborated product), } \\
\text { vinagre (elaborated } \\
\text { product) }\end{array}$ & $\mathrm{AE}, \mathrm{Bl}, \mathrm{RI}$ & $\begin{array}{l}\text { Fruit juice. Leaf. } \\
\text { Fruit peduncle } \\
\text { Stem. Whole } \\
\text { plant. }\end{array}$ & $\begin{array}{l}\text { Agrosilvopastoral management: agricultural/ } \\
\text { horticultural } \\
\text { Agrosilvopastoral management: for pig } \\
\text { slaughter } \\
\text { Cosmetic } \\
\text { Domestic: for help at home } \\
\text { Folk oral literature } \\
\text { Fuel obtaining: firewood } \\
\text { Magic and religious beliefs and practices } \\
\text { Ornamental: gardening } \\
\text { Other informations: excipient or adjuvant in } \\
\text { medicinal or food preparations } \\
\text { Repellent } \\
\text { Smoking }\end{array}$ & 35 \\
\hline $\begin{array}{l}\text { Wisteria sinensis (Sims) } \\
\text { Sweet (Fabaceae) } \\
\text { BCN30014 }\end{array}$ & Glicina & $\mathrm{AE}, \mathrm{BI}$ & Whole plant & Ornamental: gardening & 2 \\
\hline $\begin{array}{l}\text { Zantedeschia aethiopica (L.) } \\
\text { Spreng. (Araceae) } \\
\text { BCN104895 }\end{array}$ & Lliri & $\mathrm{Bl}$ & $\begin{array}{l}\text { Aerial part. } \\
\text { Whole plant }\end{array}$ & $\begin{array}{l}\text { Ornamental: bouquet elaboration } \\
\text { Ornamental: gardening }\end{array}$ & 3 \\
\hline $\begin{array}{l}\text { Zea mays L. (Poaceae) } \\
\text { BCN29830 }\end{array}$ & $\begin{array}{l}\text { Blat de les Índies, blat de } \\
\text { moro, cabellet (style and } \\
\text { stigma), maís }\end{array}$ & $\begin{array}{l}\mathrm{AE}, \mathrm{BI} \\
\mathrm{MO}, \mathrm{RI}\end{array}$ & $\begin{array}{l}\text { Bract, Epicarp, } \\
\text { Fruit. } \\
\text { Infructescence. } \\
\text { Not reported. } \\
\text { Fruit. Stem. } \\
\text { Spike. Styles } \\
\text { and stigmas. } \\
\text { Whole plant. } \\
\text { Rachis }\end{array}$ & $\begin{array}{l}\text { Agrosilvopastoral management: agricultural/ } \\
\text { horticultural } \\
\text { Fuel obtaining: firewood } \\
\text { Ludic } \\
\text { Magic and religious beliefs and practices } \\
\text { Ornamental: bouquet elaboration } \\
\text { Smoking } \\
\text { Textile: dyer } \\
\text { Textile: fibre or cloth elaboration } \\
\text { Textile: textile padding } \\
\text { Other informations }\end{array}$ & 27 \\
\hline
\end{tabular}


Table 1 Non-food and non-medicinal folk plant uses in the territories of Alt Empordà, Montseny and Ripollès (Catalonia), and Formentera and Mallorca (Balearic Islands) (Continued)

\begin{tabular}{|c|c|c|c|c|c|}
\hline $\begin{array}{l}\text { Zinnia elegans L. } \\
\text { (Asteraceae) BCN69604 }\end{array}$ & $\begin{array}{l}\text { Rascamoño (Spanish), rosa } \\
\text { mística }\end{array}$ & $\mathrm{Bl}$ & $\begin{array}{l}\text { Inflorescence. } \\
\text { Whole plant }\end{array}$ & $\begin{array}{l}\text { Agrosilvopastoral management: tools for } \\
\text { agricultural practices } \\
\text { Ornamental: bouquet elaboration } \\
\text { Ornamental: gardening }\end{array}$ & 3 \\
\hline $\begin{array}{l}\text { Ziziphus jujuba L. } \\
\text { (Rhamnaceae) BCN29822 }\end{array}$ & Gíjol (fruit), ginjoler & $\mathrm{AE}, \mathrm{Bl}, \mathrm{MO}$ & $\begin{array}{l}\text { Aerial part. } \\
\text { Fruit. Stem. } \\
\text { Whole plant }\end{array}$ & $\begin{array}{l}\text { Artisanal: musical instrument elaboration } \\
\text { Magic and religious beliefs and practices } \\
\text { Other informations: ecological information } \\
\text { Timber: boat manufacturing }\end{array}$ & 11 \\
\hline
\end{tabular}

The first two of these categories have direct links with the rural society, which has been prevalent in all the studied territories until recent times and is still important, irrespective of the intervening socioeconomic changes. Plants used in agricultural and livestock raising tasks have been and many of them continue to be utilised (see the subheading on persistence of uses). Many local handicrafts and objects for domestic uses were and are elaborated. Many kinds of baskets (Fig. 2), spoons and other kitchen implements, often made with Buxus sempervirens L. wood, or broom (Fig. 2; made with species such as Buxus sempervirens, Erica scoparia L., Mantisalca salmantica (L.) Briq. et Cavill. and Sarothamnus scoparius (L.) W.D.J.Koch -two of them with the specific epithet alluding to this object, suggesting the antiquity and relevance of this tradition-) were mentioned. The uses comprised in these two categories are also among the commonest in other European areas [11, 13, 14]. Moreover, handicraft elaboration has been reported as one of the most relevant uses in distant areas as well, e.g., [33]. Indeed, some uses belonging to other categories are also linked with rural activities. This is the case, among others, of using Datura stramonium L. as a repellent for moles, which can damage plant cultures.

The third group of uses is very common as well, since all human groups have influences of religious and/or magic beliefs. Protective plants (see the subheading on persistence of uses), or ritual ones (e.g., Laurus nobilis L., Olea europaea L. and Phoenix dactylifera L., which

Table 2 Use reports in the studied areas

\begin{tabular}{llll}
\hline Area & Use reports & $\begin{array}{l}\text { Use reports/ } \\
\text { inhabitant }\end{array}$ & $\begin{array}{l}\text { Use reports/ } \\
\text { informant }\end{array}$ \\
\hline Catalonia (CA) $^{\mathrm{a}}$ & 2335 & 0.0086 & 4.58 \\
Alt Empordà (AE) & 1014 & 0.0072 & 5.70 \\
Montseny (MO) & 942 & 0.0090 & 5.48 \\
Ripollès (RI) & 379 & 0.0147 & 2.37 \\
Balearic Islands (BI) & 1802 & 0.0021 & 6.96 \\
Formentera (FO) & 87 & 0.0075 & 3.63 \\
Mallorca (MA) & 1715 & 0.0020 & 7.30 \\
TOTAL & 4137 & 0.0036 & 5.38 \\
\hline
\end{tabular}

${ }^{a}$ Catalonia includes Alt Empordà, Montseny and Ripollès and Balearic Islands include Formentera and Mallorca are blessed in Christian Easter feasts, Fig. 3) exemplify this group of uses.

Finally, the category of ornamental plants is also universal, both in rural and urban areas, both with living or cut plants, and both for indoor and outdoor spaces. This group of uses involves classical, large-scale cultivated plants (such as Rosa sp. cultivars), but a large number of wild plants as well, such as, among many others (see Table 1), Lunaria annua L. (Fig. 3), with the aerial parts with fruiting septa of which bouquets are prepared. Many ornamental plants also have other uses; amongst them, medicinal is not rare, and in some cases the medicinal application used to be important and has decreased, but the ornamental use persists (such as, for example, in Lilium candidum L.). Decorative uses are very dynamic. The ancient traditions in this field coexist with recently adopted ones, such as using Poinsettia pulcherrima (Willd.) Graham toghether with the classical Ruscus aculeatus L. for home ornamentation at Christmas (Fig. 4).

Table 3 Use categories considered and number of reports of each one in the territories studied

\begin{tabular}{lllll}
\hline Use categories & $\mathrm{AE}$ & $\mathrm{Bl}$ & $\mathrm{MO}$ & $\mathrm{Rl}$ \\
\hline Agrosilvopastoral management & 135 & 284 & 159 & 24 \\
Artisanal & 250 & 157 & 96 & 31 \\
Cosmetic & 26 & 78 & 9 & 12 \\
Domestic & 77 & 113 & 63 & 23 \\
Folk oral literature & 16 & 106 & 64 & 10 \\
Fuel obtaining & 16 & 58 & 27 & 9 \\
Ludic & 40 & 64 & 117 & 26 \\
Magic and religious beliefs and practices & 128 & 116 & 105 & 72 \\
Ornamental & 135 & 193 & 201 & 117 \\
Other informations & 79 & 383 & 13 & 33 \\
Repellents & 20 & 95 & 3 & 11 \\
Smoking plant & 24 & 18 & 19 & 3 \\
Textile & 25 & 58 & 1 & 4 \\
Timber & 43 & 79 & 65 & 4 \\
TOTAL & 1014 & 1802 & 942 & 379 \\
\hline
\end{tabular}

$A E$ Alt Empordà, $B /$ Balearic Islands (comprising here Formentera and Mallorca), MO Montseny, RI Ripollès 


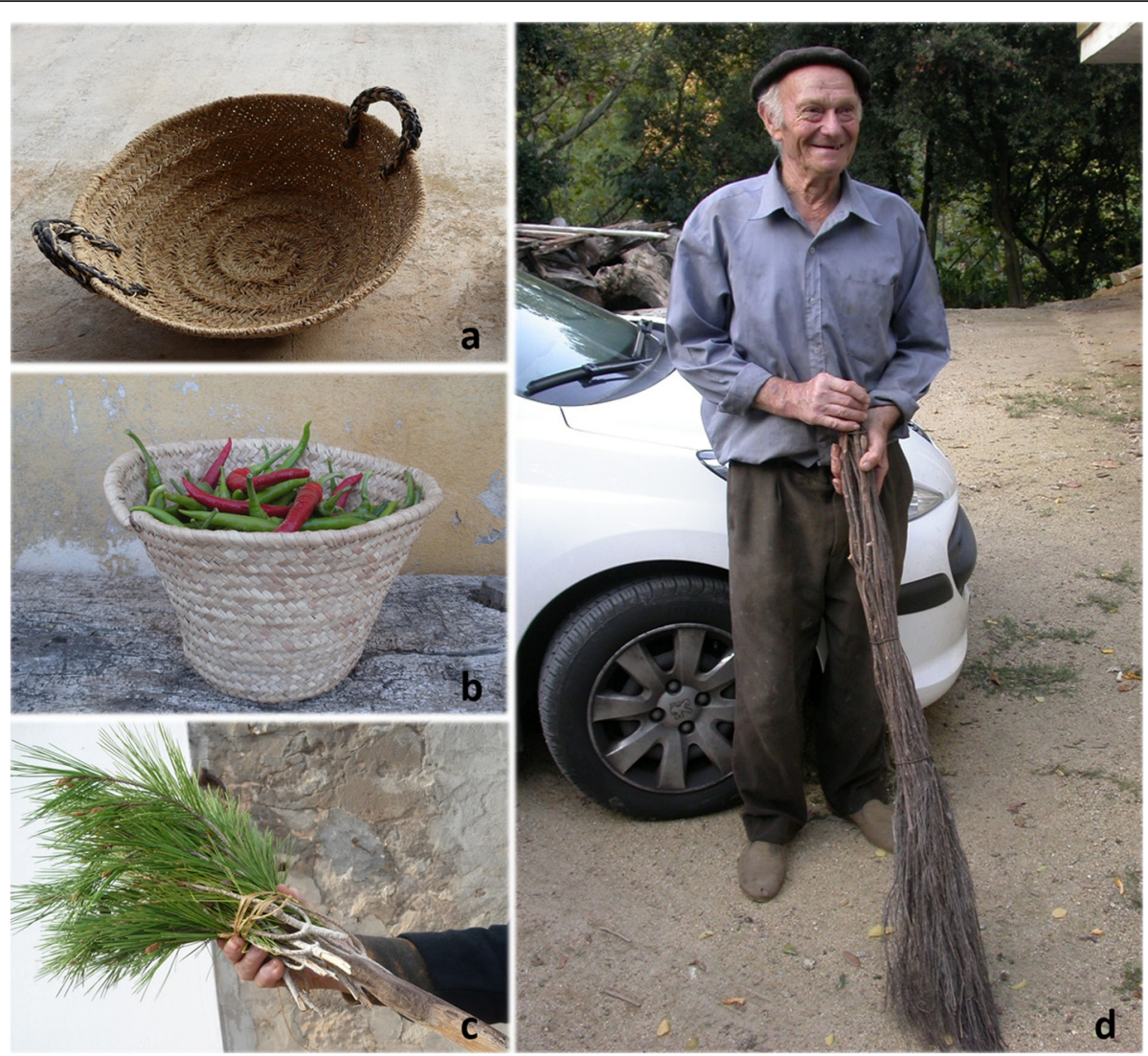

Fig. 2 Examples of basketry and broom elaboration in the territories considered: a. Formentera, Lygeum spartum; b. Mallorca, Chamaerops humilis; c. Formentera, Juniperus phoenicea, Pinus halepensis; d. Montseny, Erica scoparia

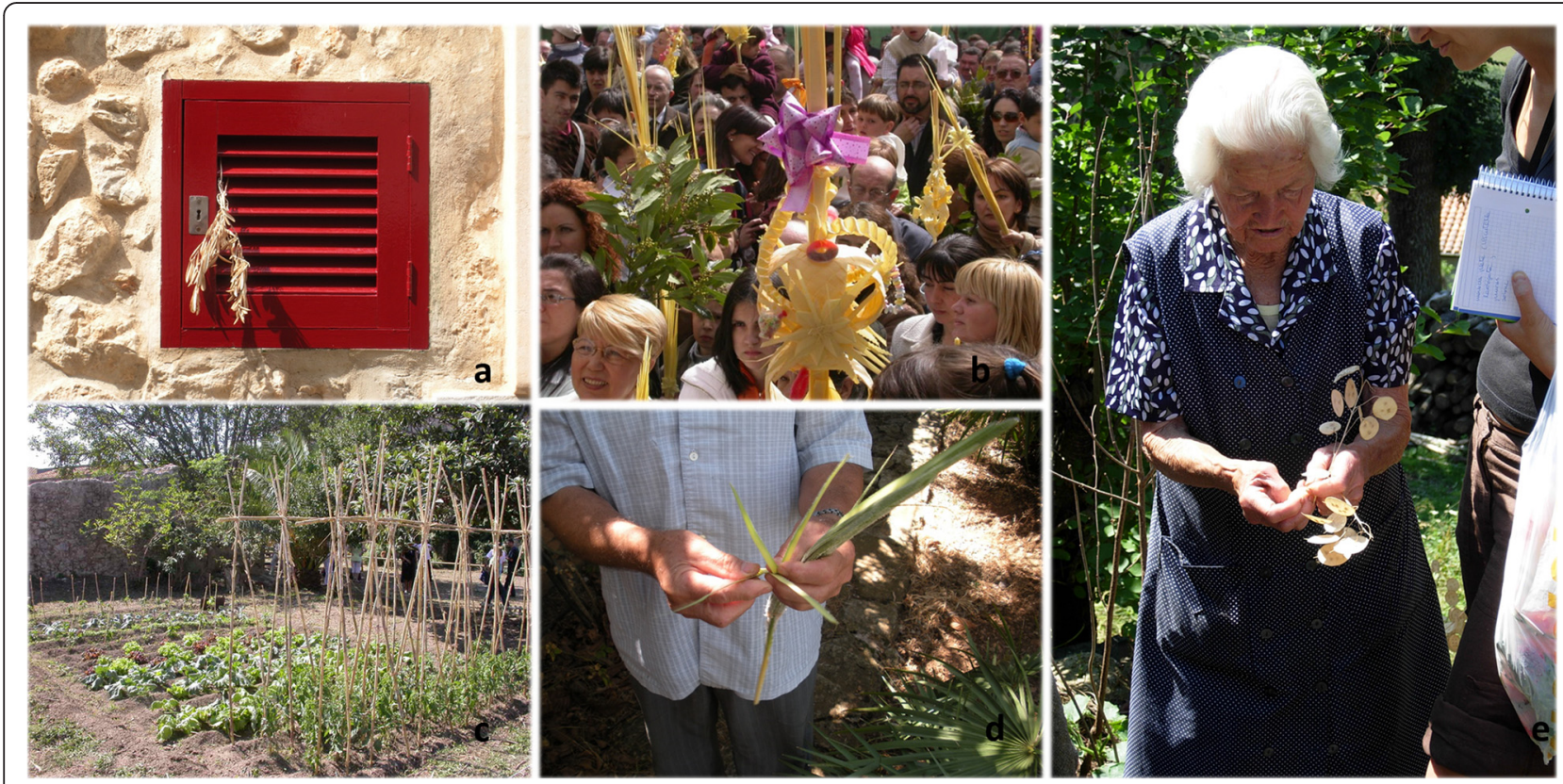

Fig. 3 Examples of artisanal, domestic and magicoreligous uses in the territories considered: a. Mallorca, Olea europaea; b. Alt Empordà, Laurus nobilis, Phoenix dactylifera; c. Alt Empordà, Arundo donax; d. Mallorca, Chamaerops humilis; e. Ripollès, Lunaria annua 


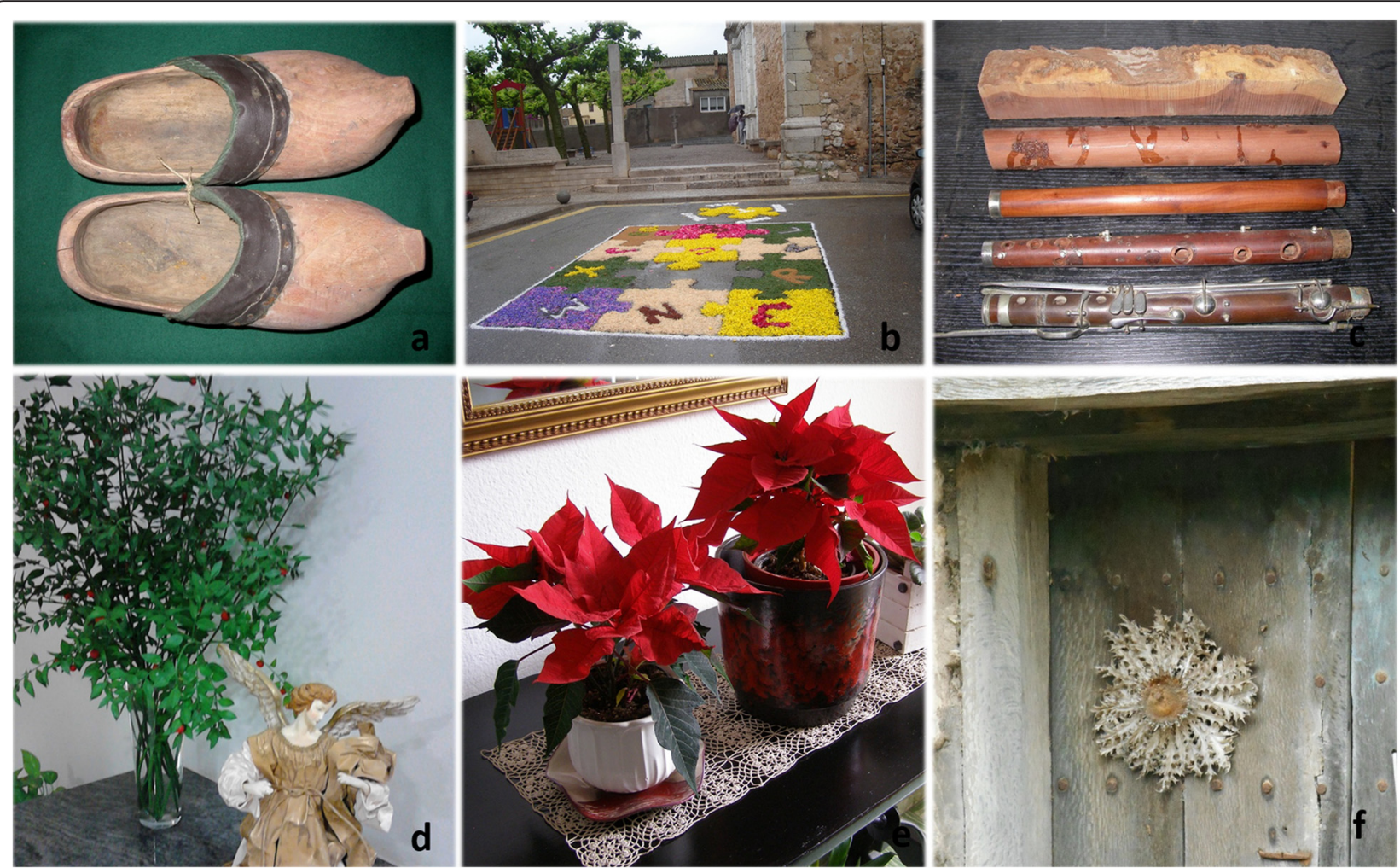

Fig. 4 Examples of artisanal, domestic and magicoreligous uses in the territories considered: a. Alt Empordà, Pinus pinea; b. Alt Empordà, several species, among which Dianthus caryophyllus, Malva sylvestris, Rosa sp., Spartium junceum and Phoenix dactylifera; c. Alt Empordà, Ziziphus jujuba; d. Montseny, Ruscus aculeatus; e. Montseny, Poinsettia pulcherrima; f. Ripollès, Carlina acanthifolia subsp. cynara

\section{Most reported taxa}

The five most reported families in the whole studied area are Poaceae (301 UR; $7.28 \%$ ), Lamiaceae (293 UR; $7.08 \%$ ), Asteraceae (290 UR; $7.01 \%)$, Rosaceae (186 UR; $4.50 \%)$ and Oleaceae (181 UR; $4.38 \%)$. All these families, except the last one, are big in terms of number of taxa and are importantly represented worldwide and, in particular, in the Mediterranean region, to which the territories considered belong. This would agree with the assessment that plant taxa common or well-represented in one area are more accessible to its inhabitants and, so, most likely used there [34]. Three of these families, Asteraceae, Lamiaceae and Rosaceae, are almost always dominant in ethnobotanical works conducted in the Catalan cultural area and in other western Mediterranean countries (see, for instance, [35] for a Catalan territory and [36, 37] for non-Catalan areas, and references contained in these papers) even if those works are focused on food and medicinal plants and the present one on other uses. The coincidence is also important (four out of the five main families of the present paper) even with work conducted in several eastern Mediterranean areas [14]. In contrast, the family Oleaceae is not very numerous in taxa, but it reached a very high number of UR mostly due to the relevance and versatility of uses of
Olea europaea (including, but not only, olive oil, utilised for many different purposes) in all Mediterranean territories. The family Poaceae is not included among the most reported in the above-quoted ethnobotanical works on food and medicinal plants, but is, again, a very large one and with some prominent plants in the kind of uses here studied. Arundo donax L. is particularly significant in this sense. This allochthonous plant, acclimated from ancient times in the areas studied, has many uses, mostly linked to agricultural practices (Fig. 3), in three of the territories (AE in Catalonia and both from $\mathrm{BI}$ ), which account for many UR.

The five most cited species in the different regions studied are presented in Table 4. Again, Olea europaea is present in the top plants list, being the most reported one in the Balearic areas considered and in one of the Catalonian territories (altogether, and logically, in the most strict Mediterranean areas among those studied) for the reasons stated in the last paragraph. The coincidence of Buxus sempervirens as highly quoted in the three Catalonian territories responds, apart from its popularity due to the high quality of its wood, to the existence of Eurosiberian zones in these continental areas, and to its practical or total absence from Balearic ones. Conversely, Chamaerops humilis (Figs. 2 and 3), much 
Table 4 Data on use reports of the most quoted plants in the territories studied

\begin{tabular}{|c|c|c|}
\hline Territory/Plant species & $\begin{array}{l}\text { Number of } \\
\text { use reports in } \\
\text { each territory }\end{array}$ & $\begin{array}{l}\text { Percentage of } \\
\text { use reports in } \\
\text { each territory }\end{array}$ \\
\hline \multicolumn{3}{|l|}{ Alt Empordà } \\
\hline $\begin{array}{l}\text { Olea europaea L. (including both subsp. } \\
\text { europaea and subsp. sylvestris (Mill.) Hegi) }\end{array}$ & 44 & 4.34 \\
\hline Arundo donax L. & 40 & 3.94 \\
\hline Erica arborea $\mathrm{L}$. & 39 & 3.85 \\
\hline Celtis australis $\mathrm{L}$. & 29 & 2.86 \\
\hline Buxus sempervirens $\mathrm{L}$. & 28 & 2.76 \\
\hline \multicolumn{3}{|l|}{ Ripollès } \\
\hline Buxus sempervirens & 28 & 7.39 \\
\hline Carlina acanthifolia (Pourr. ex DC.) Rouy & 14 & 3.69 \\
\hline Sarothamnus scoparius L. & 13 & 3.43 \\
\hline Syringa vulgaris $\mathrm{L}$. & 12 & 3.17 \\
\hline Pinus sylvestris $\mathrm{L}$. & 9 & 2.37 \\
\hline \multicolumn{3}{|l|}{ Montseny } \\
\hline Buxus sempervirens & 69 & 7.11 \\
\hline Erica scoparia L. & 30 & 3.18 \\
\hline Helichrysum stoechas (L.) Moench. & 29 & 3.08 \\
\hline Pteridium aquilinum (L.) Kuhn & 24 & 2.55 \\
\hline $\begin{array}{l}\text { Quercus ilex L. and Scirpus } \\
\text { holoschoenus L., ex aequo }\end{array}$ & 20 & 2.12 \\
\hline \multicolumn{3}{|l|}{$\begin{array}{l}\text { Balearic Islands (Mallorca and } \\
\text { Formentera) }\end{array}$} \\
\hline $\begin{array}{l}\text { Olea europaea L. (including both } \\
\text { subsp. europaea and subsp. sylvestris) }\end{array}$ & 80 & 4.44 \\
\hline Arundo donax & 57 & 3.16 \\
\hline Ocimum basilicum $\mathrm{L}$. & 47 & 2.61 \\
\hline Chamaerops humilis $\mathrm{L}$. & 46 & 2.55 \\
\hline $\begin{array}{l}\text { Pinus halepensis Mill. and Prunus dulcis } \\
\text { (Mill.) D.A.Webb., ex aequo }\end{array}$ & 41 & 2.28 \\
\hline
\end{tabular}

more present in the Balearic areas considered, and scarce or inexistent in the Catalonian ones, is, not surprisingly, highly reported in the former. Most quoted taxa are quite different from those leading the lists of top plants for medicinal or food uses in the areas considered ([28, 38] and references therein), with the exception of Olea europaea, which also plays a relevant role in the mentioned fields.

\section{Parts of plants}

The use categories object of the present paper being very diverse, almost all plant parts (and the whole plants as well) are used.

Stem (1156 UR; $27.94 \%$ ), aerial part -constituted either by all the aboveground part of the plant or by leaves and flowers together, with the stem or branch portions sustaining them- (785 UR; $18.98 \%$ ), flower (including inflorescence and flower, 337 UR; $8.15 \%$ ) and leaf (295 UR; $7.13 \%)$ have been the most reported plant parts. Frequently, the whole plant is also used (869 UR; $21.01 \%)$. Sometimes, the aerial part and the whole plant have constituted indistinguishable categories. The high number of use reports attributed to the stem is due to large and diverse kinds of artisanal uses, wood products and fuel.

\section{Comparison between the territories studied}

As stated when addressing the most recorded taxa, the territories studied share a language and culture, but differ in ecogeographical characteristics. For these comparative purposes we have put together both Balearic territories, since Formentera's dataset is not big enough to be taken into consideration.

Chi-square test showed there was an association between use categories and territories $\left(X^{2}=846.306\right.$, d.f. $=39, P<0.0001)$. Particularly, the presence of continental and insular areas leads us to suppose some differences in ethnoflora applications, possibly due to differences both in flora and in traditions. Indeed, there is also a significant difference in the use categories between continental and insular territories $\left(X^{2}=485.037\right.$, d.f. $=13, P<0.0001$ ), confirming this geographical effect.

We present in Fig. 5 the degree of coincidence in plants used in the five regions considered. Whereas the number of coincident species among three territories ranges from 43 (Balearic Islands, Montseny and Ripollès) to 76 (Alt Empordà, Balearic Islands and Montseny), this decreases to 34 when we analyse the intersection of the four areas included in this study. In any case, we believe that the amount of plants used for non-medicinal and non-food aims in all areas is rather high. These plants with a larger geographical reach are usually cosmopolitan species used in daily life in the territories of Catalan culture, such as Fagus sylvatica L., Laurus nobilis, Malva sylvestris L., Olea europaea, Papaver rhoeas L., Ruscus aculeatus, and Spartium junceum L. (Table 1).

\section{Reliability of uses recorded}

As already stated, the uses here reported are divided in a large number of categories. Irrespective of this dispersion, which is logical, since we consider here all traditional plant uses apart from food and medicinal ones, we think it is necessary to evaluate the degree of reliability of the information provided by the interviewees on these uses. A first, simple approach to this is considering the frequency of uses (see Table 1 for plants and Table 3 for use categories). According to several authors [33, 39], a use could be considered reliable if it is quoted by at least three independent informants. In most cases both the 


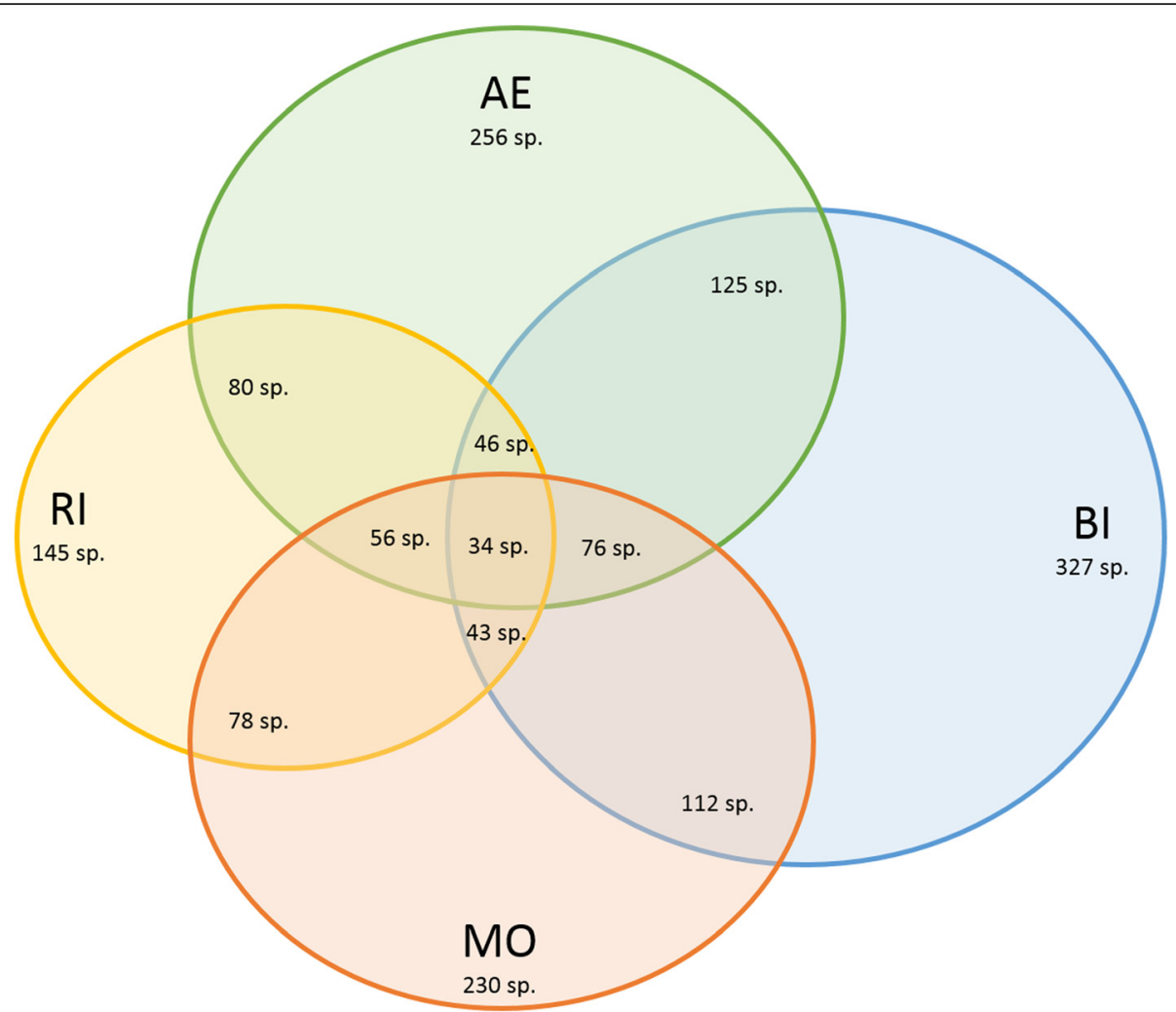

Fig. 5 Venn's diagram showing the number of uses in each territory considered and the coincidences among them. AE: Alt Empordà; BI: Balearic Isands; Mo: Montseny; Rl: Ripollès

plants and the uses have been quoted by more (usually many more) than three informants.

A finer approach is provided by the informant consensus factor $\left(F_{I C} ;[40]\right)$, defined as the number of UR minus the number of taxa divided by the number on UR minus 1. This index reflects the reliability of ethnobotanical information considering the coincidence of the informants in the same uses to be attributed to the same plants. The closer $F_{I C}$ is to its maximum value, 1 , the more consistent -thus, the more reliable- are the folk plant uses considered in the area studied. The general $\mathrm{F}_{\mathrm{IC}}$ value in this study is 0.87 and those for the two big geographical areas concerned (Catalonia and Balearic Islands) are coincidental, 0.82. To our knowledge, this is the first, or one of the very rare times, that $\mathrm{F}_{\mathrm{IC}}$ is calculated for uses other than medicinal and food ones. The absolute values can be considered rather high. Comparatively, they are higher than those calculated for medicinal uses in two Mexican and one Indian areas (ranging from 0.75 to 0.79 ; [41-43]). This means that other uses in the territories here studied are slightly more coincidental than medicinal uses in places where folk use of medicinal plants is highly practiced and considered, suggesting a high consistency and reliability of the information collected. The $F_{I C}$ values here presented are very close (although in a few cases slightly lower) to those obtained for food and medicinal plant uses in the areas object of study ([31, 35], and references therein). This testifies for the robustness of the dataset collected, confirming the relevance of these other uses of plants for the human populations practicing them.

\section{Persistence and consideration of non-food and non-medicinal folk plant uses}

If indices such as $\mathrm{F}_{\mathrm{IC}}$ permit a quantitative assessment of the non-food and non-medicinal popular plant uses, the interviews performed with a big number of informants in the five studied areas allow us to qualitatively evaluate their current situation as well. Our aprioristic idea was that, in an industrialised society, these uses, most of them not directly linked to wellness and hardly related to rural life, would be residual and not much considered, at least in part due to the processes of acculturation and erosion of traditional knowledge. Nevertheless, once considered in detail the definitely high amount of information collected, we believe that these uses are (at least still) not so marginal. We contribute here some reflections on this subject with different possibilities for the uses considered, illustrated with a few examples, and 
more similar conclusions may be deduced by considering the information of Table 1.

Undoubtedly, some of these uses have declined, in some cases nearly or completely until extinction. We can note in this category some artisanal works and most traditions linked to religious and/or magic beliefs. For instance galoshes (Fig. 4), for which the wood of several trees was employed, are no longer in use in agropastoral tasks (so, they are no longer fabricated), but the informants who used to employ them and those who used to elaborate them (one of our informants was one of the last artisans who elaborated galoshes in Catalonia, until 1980) precisely remember the details of their use and had often brought into the house some pieces as decorative elements with an added value. In the Catalan society, the same degree as in the Netherlands -where galoshes have become an identitary element, with, additionally, a big implication in merchandising and tradehas not been reached, but nowadays the few people who know how to fabricate them are often required in popular feasts to show off their former trade. Indeed, courses or workshops on these and other subjects related with traditional plant uses (e.g., dyeing) are frequently organised. Similarly, carpets elaborated with vegetal elements are no longer used in religious processions (simply because these processions do not exist currently), but in some localities in two of the areas studied (Alt Empordà, Montseny) the carpets continue to be prepared every year in the context of a popular feast (Fig. 4). In other cases, such as the elaboration of matrasses with Zea mays L. inflorescence bracts or that of sheets with Cannabis sativa L. fibres, the uses have been completely abandoned.

Even in some trades that are declining, a certain degree of vitality persists: the elaboration of musical instruments for the orchestra (named cobla) that plays typical Catalan dances (called sardana) and for other kind of traditional musical events, importantly involving Ziziphus jujuba Mill. (Fig. 4) continues to be alive, even if their artisans must have another profession to make a living.

Apart from these declining uses, some other persist almost to the same extent as always. These are uses linked to everyday life mostly in rural areas, such as those related to agricultural practices (in homegardens or elsewhere), e.g., tutoring some cultivated plants (Fig. 3). Another kind of folk plant knowledge to persist is that related to ludic aspects, including oral literature. People continue to play with plants and to use sayings and proverbs involving the vegetal world (even to inventing new ones). Additionally, some plant uses directly linked to commercial issues have also a high degree of persistence, such as those related to basketry (Fig. 1) or Christmas/New Year decoration (Fig. 4).
Finally, some popular traditions linked to plants are still in use, but have changed in some senses. As an example, some plants are hung in or near the doors of many rural houses (especially in mountain areas), as was commonly done since time immemorial, such as Carlina acanthifolia (Fig. 4). Nevertheless, no one believes nowadays (even if many people did a few decades ago) that these plants are protective for people and animals in the house. In this case, these plants now play a decorative role, with the associated interest that people know that this current use derives from a quite different ancient one. The basket elaboration mentioned in the last paragraph and similar activities, such as some related to textile, dyeing and handicraft issues, have also experienced, at least to some extent, this change. In past times, baskets, carpets, blankets, forks, spoons and other objects were elaborated with plant material simply for their use within the house, but now most such pieces produced are addressed to decorative and touristic purposes, comporting some commercial revenues.

\section{Conclusions}

The important number of species claimed to be used (552, with 4137 use reports) by the 769 informants interviewed in the territories studied for the purposes comprised in this paper show that popular knowledge on plants goes far beyond food and medicinal applications, which had been the most traditionally studied in ethnobotanical surveys, probably because they are the most apparent and the most preserved plant utilisations. This means that a large and diverse panoply of traditional uses, which are apparently secondary in our industrialised societies, such as examples as different as basket elaboration and oral literature, remains active and, when this is not so, appreciated and remembered by people.

To summarize, we believe that the data in this study show that a robust set of knowledge and practices in the field of non-food and non-medicinal plant uses persists in the European industrialised area studied, indicating that these utilisations of plants, which to date have been the object of scarce attention in ethnobotanical research, are relevant -we would say fully necessary- for a complete life, even in a modern society and even if they are not directly related to health issues. Additionally, and not forcibly negatively, although this means a reorientation in the tradition, some changes in popular uses (mostly those related to handicrafts) currently imply a certain complementary income for some people and may represent a potential for future commercial activities with economic significance in rural communities.

Further ethnobotanical studies in this currently still rather neglected field are encouraged in order to compile an important part of natural and cultural heritage not 
sufficiently considered up to now, and to assess what actions are needed to preserve this knowledge, particularly in cases of dangerously declining real current practice.

\section{Abbreviations}

$\mathrm{AE}$, Alt Empordà district; $\mathrm{BC}$, Herbarium of Botanical Institute of Barcelona; $B C N$, Herbarium of the Centre de Documentació de Biodiversitat Vegetal, Universitat de Barcelona; Bl, Balearic Islands; CA, Catalonia; Fic, Informant consensus factor; FO, Formentera island; MA, Mallorca island; MO, Montseny massif; RI, Ripollès district; UR, Use reports

\section{Acknowledgements}

We thank all the informants for having shared with us their time and their in-depth knowledge on biodiversity use and management. We are grateful to Samuel Pyke (Jardí Botànic de Barcelona) for his improvement of the English language, to Rosa Trigell (Blommor Floristes) for plant material supply and to Josep Vicens (Universitat de Barcelona) for his help in herbarium material management. EC and AG benefited from predoctoral contracts of the Spanish ministries in charge of education. This research has been partially funded by the municipal council of Figueres (IX Beca de recerca "Ciutat de Figueres"), the Catalan government (projects 2009SGR439, 2009ACOM00012, 2009ACOM00013 and 2014SGR514) and the Spanish government (project CSO2014-59704-P).

\section{Funding}

See acknowledgements

\section{Availability of data and materials}

Not applicable

\section{Authors' contributions}

All authors participated in the design of the research and in data collection AG and TG carried out the statistical analyses. TG and JV coordinated the study and wrote the first draft of the manuscript, to which the other authors then contributed. All authors read and approved the final manuscript.

\section{Competing interests}

The authors declare that they have no competing interests.

\section{Consent for publication}

The authors give their consent for publication of this manuscript.

\section{Ethics approval and consent to participate}

All the authors agree with the manuscript and consent to participate in it. Concerning the informants, they gave the informed consent (see Methods section).

\section{Author details}

${ }^{1}$ Laboratori de Botànica - Unitat associada CSIC, Facultat de Farmàcia, Universitat de Barcelona, Av. Joan XXIII s/n, 08028 Barcelona, Catalonia, Spain. ${ }^{2}$ Institut Botànic de Barcelona (IBB-CSIC-ICUB), Passeig del Migdia s/n. Parc de Montjuïc, 08038 Barcelona, Catalonia, Spain.

Received: 6 February 2016 Accepted: 7 June 2016

Published online: 17 June 2016

\section{References}

1. Harshberger JW. Purposes of ethnobotany. Bot Gazet. 1896:21:146-54

2. Pardo de Santayana M, Pieroni A, Puri R. Ethnobotany in the new Europe. People, health and wild plant resources. New York-Oxford: Berghahn Books; 2010.

3. Portères R. Cours d'Ethno-botanique et Ethno-zoologie (1969-1970). Volume I, Ethno-botanique générale. Paris: Muséum National d'Histoire Naturelle (Laboratoire d'Ethno-botanique et Ethno-zoologie), Faculté des Lettres (Institut d'Ethnologie); 1970.

4. Barrau J. L'Ethnobotanique au carrefour des sciences naturelles et des sciences humaines. Bull Soc Bot France. 1971;118:237-48.

5. Gispert M, Coutiño B, Díaz A. Contemporary challenges of ethnobotany. In: Albuquerque UP, Hanazaki N, editors. Recent developments and case studies in ethnobotany. Recife: Sociedade Brasileira de Etnobiologia
\& Núcleo de Publicações em Ecologia e Etnobotânica Aplicada; 2010. p. 13-32.

6. Ember CR, Ember M. Antropología cultural. 8th ed. Madrid: Prentice Hall; 1997.

7. Heinrich M. Ethnobotany and its Role in Drug Development. Phytother Res. 2000;14:479-88.

8. Carvalho AM, Pardo-de-Santayana M, Morales R. Traditional knowledge of basketry practices in a Northeastern region of Portugal. In: Ertug F, editor. Proceedings of the IVth International Congress of Ethnobotany (ICEB 2005). 2006. p. 335-8.

9. González JA, García-Barriuso M, Ramírez-Rodríguez R, Bernardos S, Amich F. Plants used in folk cosmetics and hygiene in the Arribes del Duero Natural Park (western Spain). Lazaroa. 2012;33:9-18.

10. Rigat M, Vallès J, D'Ambrosio U, Gras A, Iglésias J, Garnatje T. Plants with topical uses in the Ripollès district (Pyrenees, Catalonia, Iberian Peninsula): ethnobotanical survey and pharmacological validation in the literature. J Ethnopharmacol. 2015;164:162-79.

11. Salerno G, Guarrera PM, Caneva G. Agricultural, domestic and handicraft folk uses of plants in the Tyrrhenian sector of Basilicata (Italy). J Ethnobiol Ethnomed. 2005;1:2.

12. Guarrera PM. Household dyeing plants and traditional uses in some areas of Italy. J Ethnobiol Ethnomed. 2006;2:9.

13. Guarrera PM. Handicrafts, handlooms and dye plants in the Italian folk traditions. Indian J Tradit Knowl. 2008;7:67-9.

14. Nedelcheva A, Dogan Y, Obratov-Petkovic D, Padure IM. The Traditional Use of Plants for Handicrafts in Southeastern Europe. Hum Ecol. 2011;39:813-28.

15. Savo V, La Rocca A, Caneva G, Rapallo F, Cornara L. Plants used in artisanal fisheries on the Western Mediterranean coasts of Italy. J Ethnobiol Ethnomed. 2013;9:9.

16. Fajardo J, Verde A, Rivera D, Obón C, Leopold S. Traditional craft techniques of sparto grass (Stipa tenacissima L.) in Spain. Econ Bot. 2015;69:370-6.

17. Pardo de Santayana M, Morales R, Aceituno L, Molina M. Inventario español de los conocimientos tradicionales relativos a la Biodiversidad. Primera fase. Introducción, metodología y fichas. Madrid: Ministerio de Agricultura, Alimentación y Medio Ambiente; 2014.

18. Vallès J, D'Ambrosio U, Gras A, Parada M, Serrasolses G, Garnatje T. Una denominació recent per a una ciència antiga. Algunes consideracions sobre l'etnobotànica i la seva situació a Catalunya. Rev Girona. 2015;290:80-3.

19. Bonet MÀ, Vallès J. Etnobotànica del Montseny: plantes amb usos no medicinals ni alimentaris. Monogr Serv Parcs Nat Diput Barcelona. 2002;33:25-33.

20. Parada M, Bonet MÀ, Vallès J. Usos populars de les plantes i gestió del paisatge: alguns resultats d'una recerca etnobotànica a l'Alt Empordà. Ann Inst Est Empordanesos. 2007:40:745-59.

21. IDESCAT. Institut d'Estadística de Catalunya. http://www.idescat.cat. Accessed 11 Dec 2015.

22. Bonet MÀ. Estudi etnobotànic del Montseny: Universitat de Barcelona; 2001. PhD thesis.

23. IBESTAT. Institut d'Estadística de les Illes Balears. http://ibestat.caib.es/ibestat/inici. Accessed 11 Dec 2015.

24. Goodman LA. Snowball sampling. Ann Math Stat. 1961;32:148-70.

25. International Society of Ethnobiology. International Society of Ethnobiology Code of Ethics (with 2008 additions). http://ethnobiology.net/code-of-ethics. Accessed 28 Dec 2015.

26. Pujadas JJ, Comas D, Roca J. Etnografia. Barcelona: Universitat Oberta de Catalunya; 2004.

27. de Bolòs O, Vigo J, Masalles R, Ninot J. Flora manual dels Països Catalans. 3rd ed. Barcelona: Editorial Pòrtic; 2005.

28. APG III. An update of the Angiosperm Phylogeny Group classification for the orders and families of flowering plants: APGIII. Bot J Linn Soc. 2009;161:105-21.

29. Cook F. Economic Botany Data Collection Standard. Royal Botanic Gardens: Kew; 1995.

30. Parada M, Carrió E, Bonet MÀ, Vallès J. Ethnobotany of the Alt Empordà region (Catalonia, Iberian Peninsula). Plants used in human traditional medicine. J Ethnopharmacol. 2009:124:609-18.

31. Carrió E, Rigat M, Garnatje T, Mayans M, Parada M, Vallès J. Plant ethnoveterinary practices in two Pyrenean territories of Catalonia (Iberian peninsula) and in two areas of the Balearic Islands, and comparison with ethnobotanical uses in human medicine. Evid-Based Compl Alternat Med. 2012;2012:896295. ID 896295. 
32. Vallès J, Veny J, Vigo J, Bonet MÀ, Julià MA, Villalonga JC. Noms de plantes. Corpus de fitonímia catalana. Barcelona: Termcat - Centre de Terminologia \& Universitat de Barcelona; 2014.

33. Sop TK, Oldeland J, Bougnounou F, Shmiedel U, Thiombiano A. Ethnobotanical knowledge and valuation of woody plants species: a comparative analysis of three ethnic groups from the sub-Sahel of Burkina Faso. Environ Dev Sustain. 2012;14:627-49.

34. Johns T, Kokwaro JO, Kimanani EK. Herbal remedies of the Luo of Siaya district, Kenya: establishing quantitative criteria for consensus. Econ Bot. 1990;44:369-81.

35. Rigat M, Bonet MÀ, Garcia S, Garnatje T, Vallès J. Ethnobotanical studies in the high river Ter valley (Pyrenees, Catalonia, Iberian Peninsula). Non-crop food vascular plants and crop food plants with medicinal properties. Ecol Food Nutr. 2009;48:303-26.

36. Pieroni A, Quave CL, Santoro RF. Folk pharmaceutical knowledge in the territory of the Dolomiti Lucane, inland southern Italy. J Ethnopharmacol. 2004;95:373-84

37. González-Tejero MR, Casares-Porcel M, Sánchez-Rojas CP, Ramiro-Gutiérrez JM, Molero-Mesa J, Pieroni A, Giusti ME, Censorii E, de Pasquale C, Della A, Paraskeva-Hadijchambi D, Hadjichambis A, Houmani Z, El-Demerdash M, El-Zayat M, Hmamouchi M, El-Johrig S. Medicinal plants in the Mediterranean area: synthesis of the results of the project RUBIA. J Ethnopharmacol. 2007;116:341-57.

38. Rigat M, Bonet MÀ, Garcia S, Garnatje T, Vallès J. Studies on pharmaceutical ethnobotany in the high river Ter valley (Pyrenees, Catalonia, Iberian Peninsula). J Ethnopharmacol. 2007;113:267-77.

39. Le Grand A, Wondergem PA. Les phytothérapies anti-infectieuses de la forêt-savane, Sénégal, Afrique Occidentale Un inventaire. Journal of Ethnopharmacol. 1987;21:109-25.

40. Trotter RT, Logan MH. Informant consensus: a new approach for identifying potentially effective medicinal plants. In: Etkin NL, editor. Plants in Indigenous Medicine and Diet, Behavioural Approaches. Bredford Hills: Redgrave Publishing Company; 1986. p. 91-112.

41. Heinrich M, Ankli A, Frei B, Weimann C, Sticher O. Medicinal plants in Mexico: Healers' consensus and cultural importance. Soc Sci Med. 1998;47: 1859-71.

42. Leonti M, Vibrans $\mathrm{H}$, Sticher $\mathrm{O}$, Heinrich M. Ethnopharmacology of the Popoluca, Mexico: an evaluation. J Pharm Pharmacol. 2001;12:1653-69.

43. Ragupathy S, Steven NG, Maruthakkutti M, Velusamy B, UI-Huda MM Consensus of the'Malasars' traditional aboriginal knowledge of medicinal plants in the Velliangiri holy hills India. J Ethnobiol Ethnomed. 2008;4:8.

\section{Submit your next manuscript to BioMed Central and we will help you at every step:}

- We accept pre-submission inquiries

- Our selector tool helps you to find the most relevant journal

- We provide round the clock customer support

- Convenient online submission

- Thorough peer review

- Inclusion in PubMed and all major indexing services

- Maximum visibility for your research

Submit your manuscript at www.biomedcentral.com/submit

) Biomed Central 